\title{
Long term photometric monitoring with the Mercator telescope ${ }^{\star}$
}

\section{Frequencies and mode identification of variable O-B stars ${ }^{\star \star}$}

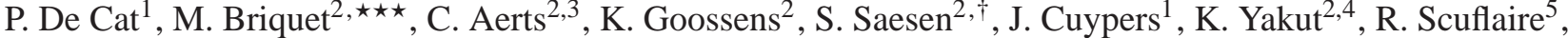 \\ M.-A. Dupret ${ }^{6}$, K. Uytterhoeven ${ }^{2,7,8}$, H. Van Winckel ${ }^{2}$, G. Raskin ${ }^{2,8}$, G. Davignon ${ }^{2,8}$, L. Le Guillou ${ }^{2,8}$, \\ R. Van Malderen ${ }^{2}$, M. Reyniers ${ }^{2, \star \star \star}$, B. Acke ${ }^{2, \star \star \star}$, W. De Meester ${ }^{2}$, J. Vanautgaerden ${ }^{2}$, B. Vandenbussche ${ }^{2}$,
}

T. Verhoelst ${ }^{2, \star \star \star}$, C. Waelkens ${ }^{2}$, P. Deroo ${ }^{2}$, K. Reyniers ${ }^{2}$, M. Ausseloos ${ }^{2}$, E. Broeders ${ }^{2}$, J. Daszyńska-Daskiewicz ${ }^{2,9}$, J. Debosscher ${ }^{2}$, S. De Ruyter ${ }^{2,10}$, K. Lefever ${ }^{2}$, G. Decin ${ }^{2}$, K. Kolenberg ${ }^{2,11}$, A. Mazumdar,12, C. Van Kerckhoven ${ }^{2}$, J. De Ridder ${ }^{2, \star \star \star}$, R. Drummond ${ }^{2}$, C. Barban ${ }^{2,6}$, E. Vanhollebeke ${ }^{2}$, T. Maas ${ }^{2}$, and L. Decin ${ }^{2, \star \star \star}$

\footnotetext{
1 Koninklijke Sterrenwacht van België, Ringlaan 3, 1180 Brussel, Belgium e-mail: peter@oma.be

2 Instituut voor Sterrenkunde, K.U. Leuven, Celestijnenlaan 200 B, 3001 Leuven, Belgium

3 Department of Astrophysics, Radboud University Nijmegen, 6500 GL Nijmegen, The Netherlands

4 Department of Astronomy and Space Sciences, Faculty of Science, University of Ege, 35100 İzmir, Turkey

5 Institut d'Astrophysique et de Géophysique, Université de Liège, Allée du Six Août 17, 4000 Liège, Belgium

6 Observatoire de Paris, LESIA, CNRS UMR 8109, 92195 Meudon, France

7 INAF - Osservatorio Astronomico di Brera, via E. Bianchi 46, 23807 Merate, Italy

8 MERCATOR Telescope, Calle Alvarez de Abreu 70, 38700 Santa Cruz de La Palma, Spain

9 Instytut Astronomiczny, Universytet Wroclawski, Kopernika 11, 51-622 Wroclaw, Poland

10 Sterrenkundig Observatorium, Universiteit Gent, Krijgslaan 281-S9, 9000 Gent, Belgium

11 Institut für Astronomie, Universität Wien, Türkenschanzstrasse 17, 1180 Wien, Austria

12 Astronomy Department, Yale University, PO Box 208101, New Haven, CT 06520-8101, USA
}

Received 7 August 2006 / Accepted 5 October 2006

\begin{abstract}
Aims. We selected a large sample of O-B stars that were considered as (candidate) slowly pulsating B, $\beta$ Cep, and Maia stars after the analysis of their HIPPARCOS data. We analysed our new seven passband GENEVA data collected for these stars during the first three years of scientific operations of the MERCATOR telescope. We performed a frequency analysis for 28 targets with more than 50 high-quality measurements to improve their variability classification. For the pulsating stars, we tried both to identify the modes and to search for rotationally split modes.

Methods. We searched for frequencies in all the GENEVA passbands and colours by using two independent frequency analysis methods and we applied a $3.6 \mathrm{~S} / \mathrm{N}$-level criterion to locate the significant peaks in the periodograms. The modes were identified by applying the method of photometric amplitudes for which we calculated a large, homogeneous grid of equilibrium models to perform a pulsational stability analysis. When both the radius and the projected rotational velocity of an object are known, we determined a lower limit for the rotation frequency to estimate the expected frequency spacings in rotationally split pulsation modes.

Results. We detected 61 frequencies, among which 33 are new. We classified 21 objects as pulsating variables $(7$ new confirmed pulsating stars, including 2 hybrid $\beta$ Cep/SPB stars), 6 as non-pulsating variables (binaries or spotted stars), and 1 as photometrically constant. All the Maia candidates were reclassified into other variability classes. We performed mode identification for the pulsating variables for the first time. The most probable $\ell$ value is $0,1,2$, and 4 for $1,31,9$, and 5 modes, respectively, including only 4 unambiguous identifications. For 7 stars we cannot rule out that some of the observed frequencies belong to the same rotationally split mode. For 4 targets we may begin to resolve close frequency multiplets.
\end{abstract}

Key words. stars: early-type - stars: variables: general - stars: oscillations

\section{Introduction}

The MERCATOR telescope is a 1.2-m telescope located on the Roque de los Muchachos observatory in La Palma (Spain). Since

* Based on observations collected with the P7 photometer attached to the Flemish 1.2-m MERCATOR telescope situated at the Roque de los Muchachos observatory on La Palma (Spain).

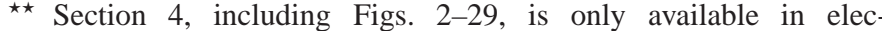
tronic form at http://www.aanda.org, and Tables 2 and 3 are only available in electronic form at the CDS via anonymous ftp to cdsarc.u-strasbg.fr $(130.79 .128 .5)$ or via http://cdsweb.u-strasbg.fr/cgi-bin/qcat?]/A+A/463/243

$\star \star \star$ Postdoctoral Fellow of the Fund for Scientific Research, Flanders

Research Assistant of the Fund for Scientific Research, Flanders the start of scientific observations in May 2001, this telescope has been intensively used to observe variable $\mathrm{O}, \mathrm{B}, \mathrm{A}$, and $\mathrm{F}$ main sequence stars with the P7 photometer, providing quasisimultaneous observations in the 7 passbands of the GENEVA photometric system. The advantages of the use of the GENEVA photometric system are threefold: (1) the calibration of the system allows the determination of stellar parameters like the effective temperature and the surface gravity, (2) the observations are always obtained and reduced in the same way which is ideal to detect light-variability, and hence to search for pulsations, and (3) because the system consists of 7 filters, it provides the information necessary to determine the degree $\ell$ of the observed pulsation modes. Therefore, our long-term study enables 


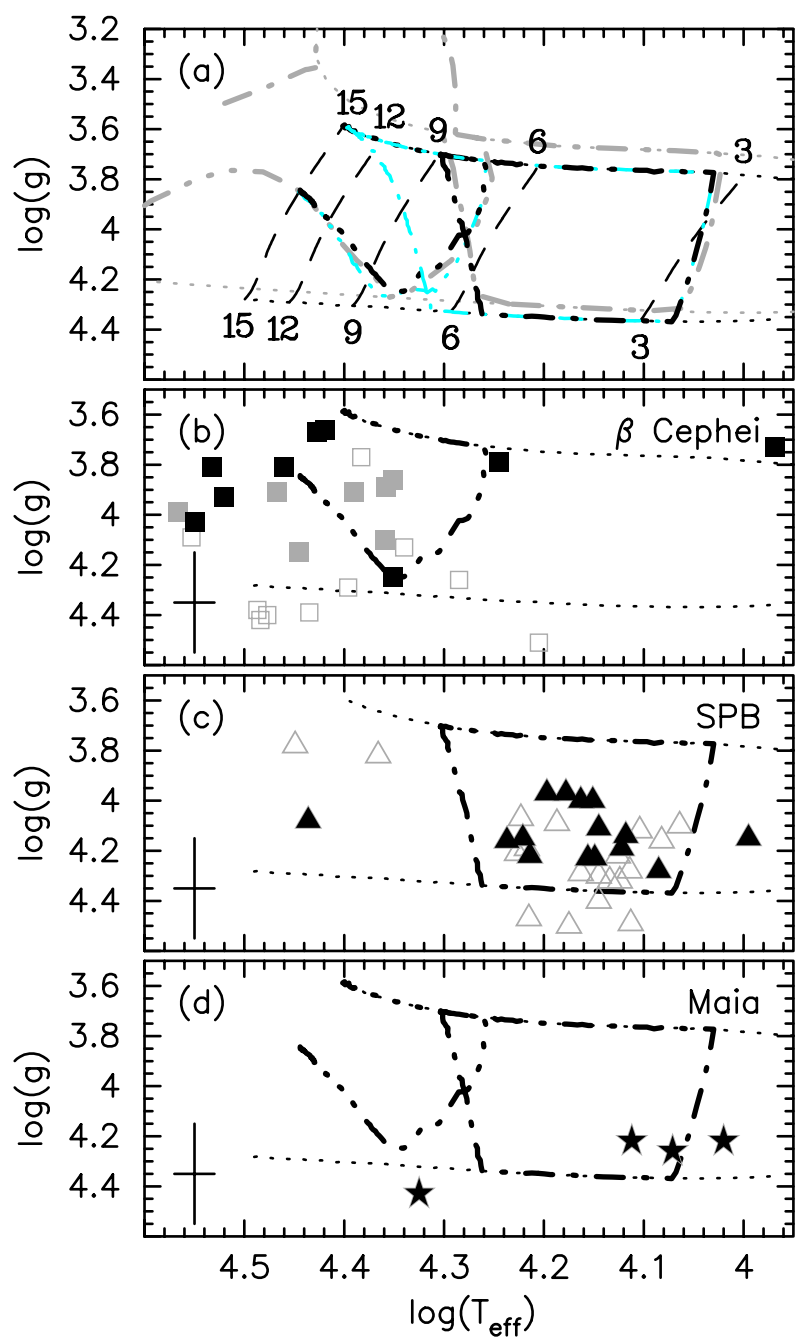

Fig. 1. Position in the $\left(\log \left(T_{\text {eff }}\right), \log (g)\right)$ diagram of the variable O-B stars with MERCATOR data. In each panel, the lower and upper dotted lines show the ZAMS and TAMS, respectively. The dashed lines denote evolution tracks for stars with $M=15,12,9,6$, and $3 M_{\odot}$. The dashdot-dot-dotted and dash-dotted lines represent the theoretical instability strips for $\beta$ Cep and SPB modes, respectively, with a frequency between 0.25 and $25 \mathrm{~d}^{-1}$ and $\ell \leq 2$ (black) / 4 (cyan) computed for the main sequence models described in Sect. 3.2 with the non-adiabatic code MAD (Dupret 2001) (the colour representation is only available in the online version of the paper). In panel a), the ZAMS, TAMS, and theoretical instability strips derived by Pamyatnykh (1999) are given in grey. The stars classified as (suspected) $\beta$ Ceps, SPBs and Maias at the start of our project in 2001, are given with squares, triangles, and stars in panel b), c), and d), respectively. Typical error bars are given in the lower left parts. The objects with more than 50 observations are given with full symbols. The objects given in black are discussed in this paper.

us to classify northern objects into different classes of variable stars, and hence to contribute to the determination of the population rates in the northern hemisphere. Moreover, long time series of high-quality, quasi-simultaneous multicolour observations allow accurate class-definitions used in supervised classification of large data-sets (e.g. earth-based projects like OGLE and MACHO, or the future satellite missions COROT and GAIA; Debosscher et al. 2006). Long-term observations also help to reach the level of accuracy on the amplitudes of the observed variations in the GENEVA $U, B_{1}, B, B_{2}, V_{1}, V, G$ bands necessary to allow an unambiguous determination of the degree $\ell$ of the excited pulsation modes. A reliable identification of the excited modes opens the door to asteroseismic modelling. The importance of dedicated long-term campaigns is well-illustrated by the case of HD 129929: after 21 years of single-site monitoring, Aerts et al. (2003) found the first strong evidence for nonrigid rotation and convective core overshoot in a $\beta$ Cep star based on asteroseismic modelling of six frequencies, among which are components of two frequency multiplets.

Our first results, obtained after 18 months of observations were presented by De Cat et al. (2004b) and De Ridder et al. (2004). We now present results after 3 years of collecting data. This paper deals with the classification, frequency analysis and mode identification of the variable O-B stars. Cuypers et al. (in preparation) focus on a similar study of variable A and F stars.

So far, 64 variable O-B stars have been observed with the MERCATOR telescope. At the start of our long-term project in 2001 , some of our targets were classified as suspected $\beta$ Cep stars $(\beta$ Ceps; main sequence B $0-3$ stars pulsating in low-order, low-degree $p / g$-modes with periods of 3-8 h; e.g. Stankov \& Handler 2005), slowly pulsating B stars (SPBs; main sequence B 3-B 9 stars pulsating in high-order, low-degree $g$-modes with periods of 0.5-3 d; e.g. Waelkens 1991) or Maia stars (Maias; main sequence B 7-A 2 stars showing variations with periods of 2-8 h; e.g. Struve 1955). This classification was based on the photometric observations gathered with the satellite mission HIPPARCOS. Other objects were already known as confirmed $\beta$ Ceps or SPBs. Since pulsation theory does not predict unstable modes for stars situated along the main sequence between the SPBs and the $\delta$ Scuti stars, the existence of the Maias as a separate group of variables is still disputable (e.g. Scholz et al. 1998; Aerts \& Kolenberg 2005).

There are 35 targets observed at more than 50 epochs. For the well-known $\beta$ Ceps HD 163472 (V2052 Oph), HD 165174 (V986 Oph), HD 214993 (12 Lac = DD Lac), and HD 216916 $(16 \mathrm{Lac}=\mathrm{ENLac})$, the MERCATOR telescope was included in multi-site campaigns. Their results are discussed elsewhere (Handler et al. 2006; Handler et al., in preparation). We dedicated separate papers to the multi-periodic $\beta$ Ceps HD 203664 (Aerts et al. 2006) and HD 21803 (KP Per; Saesen et al., in preparation), whose MERCATOR observations already allow us to begin asteroseismic modelling, and to HD 180642 (Aerts et al., in preparation), which was selected as a target for the COROT satellite. The MERCATOR data of the remaining 28 objects with more than 50 high-quality observations are analysed in this paper.

\section{Description of the data}

The use of the GENEVA photometric system offers some advantages essential for a statistically relevant study of variable stars. The MERCATOR telescope and the P7 photometer are always operated in the same way by the same group of astronomers, so that sustained observations in homogeneous conditions can be performed. The observations are planned in such a way that the program stars are observed at an almost constant air mass during the night. In our project, the air mass interval $v_{z} \in[1.05,1.15]$ was generally used. For the stars in our project, the exposure times are typically $3 \mathrm{~min}$. The planned sampling rates for the (candidate) $p$ - and $g$-mode pulsators are a few times each hour and day, respectively. Every hour, a pair of standard stars with distinct spectral types in this air mass interval is observed to determine the extinction coefficients. The data acquisition and reduction has been centralised in Geneva since the early history of the system. For a full description of the reduction methods, we refer to Rufener (1986) and Burki et al. (1995). 
Table 1. Overview of the 28 targets discussed in this paper. The stars classified as (suspected) $\beta$ Ceps, SPBs and Maias at the start of our project in 2001 are given in the top, middle, and bottom part, respectively. For each object, we give: the HD number, the HIP number, the Johnson $V$ magnitude $m_{V}^{1}$, the spectral type $S p T^{1}$, a binarity indication $(\mathrm{VB}=$ visual binary, $\mathrm{SB} 1=$ single-lined spectroscopic binary, $\mathrm{SB} 2=$ double-lined spectroscopic binary), the number of MERCATOR observations $N$ used during the analysis, the corresponding total time span $\triangle T$ (days), the effective temperature $\log \left(T_{\text {eff }}\right)^{2}( \pm 0.020 \mathrm{dex})$, the surface gravity $\log (g)^{2}( \pm 0.20 \mathrm{dex})$, the mass $M\left(M_{\odot}\right)$, the radius $R\left(R_{\odot}\right)$, the $\operatorname{luminosity} \log \left(L / L_{\odot}\right)(\operatorname{dex})$, the age $\left(10^{6}\right.$ years $)$, the fraction of the main sequence lifetime $f r a c_{\mathrm{MS}}$, the projected rotation velocity $v_{\mathrm{eq}} \sin i\left(\mathrm{~km} \mathrm{~s}^{-1}\right)$, and the projected rotation frequency $f_{\Omega} \sin i=v_{\text {eq }} \sin i / 2 \pi R\left(\mathrm{~d}^{-1}\right)$. The values of $T_{\text {eff }}$ and $\log (g)$ given in italics are found by extrapolation out of the calibration tables. These results should be treated with caution, as well as those for the binaries. Error estimates (if available) are given between brackets in units of the last decimal.

\begin{tabular}{|c|c|c|c|c|c|c|c|c|c|c|c|c|c|c|c|}
\hline$\overline{\mathrm{HD}}$ & $\overline{\mathrm{HIP}}$ & $m_{V}$ & SpT & binarity & $\bar{N}$ & $\overline{\Delta T}$ & $\log \left(T_{\text {eff }}\right)$ & $\log (g)$ & $\bar{M}$ & $R$ & $\log \left(L / L_{\odot}\right)$ & age & frac $_{\mathrm{MS}}$ & $v_{\text {eq }} \sin i$ & $f_{\Omega} \sin i$ \\
\hline 13745 & 10541 & 7.9 & O9.7 II & & 158 & 1010 & 4.550 & 4.03 & & & & & & $260^{9}$ & \\
\hline 13831 & 10615 & 8.3 & B0 IIIp & & 80 & 701 & 4.520 & 3.93 & & & & & & $167^{8}$ & \\
\hline 14053 & & 8.5 & B1 II & & 64 & 374 & 4.427 & 3.67 & 14.1(9) & $8.0(18)$ & $4.44(13)$ & $10.2(5)$ & $0.86(11)$ & $70^{\circ}$ & $0.17(4)$ \\
\hline 19374 & 14514 & 6.1 & $\mathrm{~B} 1.5 \mathrm{~V}$ & & 51 & 1010 & 4.351 & 4.25 & $8.3(12)$ & $3.9(9)$ & $3.54(25)$ & $9(4)$ & $0.32(26)$ & 5 & $0.025(6)$ \\
\hline 89688 & 50684 & 6.6 & B3.2 IV & & 232 & 762 & 4.245 & 3.79 & $6.5(10)$ & $5.1(12)$ & $3.34(26)$ & $40(6)$ & $0.89(11)$ & $215^{5}$ & $0.83(20)$ \\
\hline 149881 & 81362 & 7.0 & B0.5 III & SB1 & 402 & 744 & 4.460 & 3.81 & $14.4(6)$ & $6.7(12)$ & $4.41(10)$ & $8(1)$ & $0.69(14)$ & & \\
\hline 180968 & 94827 & 5.5 & B0.5 IV & VB & 205 & 472 & 4.419 & 3.66 & $14.0(10)$ & $8.2(21)$ & $4.44(15)$ & $10.7(5)$ & $0.89(12)$ & $27 \overbrace{}^{5}$ & $0.65(17)$ \\
\hline 214680 & 111841 & 4.9 & $\mathrm{O} 9 \mathrm{v}$ & VB & 275 & 70 & 4.532 & 4.10 & & & & & & $35^{4}$ & \\
\hline 217782 & 113788 & 5.1 & A3 Vn & VB & 345 & 130 & 3.969 & 3.73 & $2.6(2)$ & $3.2(4)$ & $1.83(14)$ & 393(48) & $0.94(7)$ & $195^{8}$ & $1.20(15)$ \\
\hline 1976 & 1921 & 5.6 & $\overline{\mathrm{B} 5 \mathrm{IV}}$ & $\mathrm{VB}, \mathrm{SB} 1$ & 235 & 1147 & 4.197 & 3.97 & $5.1(10)$ & $4.0(13)$ & $2.93(33)$ & $57(7)$ & $0.76(21)$ & $140^{3}$ & $0.69(22)$ \\
\hline 3379 & 2903 & 5.9 & B2.5 IV & & 136 & 417 & 4.237 & 4.16 & $5.4(9)$ & $3.3(10)$ & $2.93(32)$ & $29(8)$ & $0.45(33)$ & $55^{5}$ & $0.33(10)$ \\
\hline 21071 & 15988 & 6.1 & B7 v & & 233 & 1140 & 4.156 & 4.23 & $4.0(6)$ & $2.7(7)$ & $2.43(28)$ & $54(17)$ & $0.39(30)$ & $62^{3}$ & $0.45(12)$ \\
\hline 24094 & 18151 & 8.4 & B8 & & 59 & 1047 & 4.436 & 4.08 & $12.5(25)$ & $5.4(19)$ & $4.15(34)$ & $6(2)$ & $0.44(32)$ & & \\
\hline 25558 & 18957 & 5.3 & B3 V & & 148 & 1046 & 4.214 & 4.22 & $4.9(7)$ & $3.0(8)$ & $2.76(29)$ & 33(9) & $0.40(29)$ & $22^{3}$ & $0.14(4)$ \\
\hline 28114 & 20715 & 6.1 & B6 IV & & 162 & 811 & 4.163 & 4.00 & $4.5(8)$ & $3.6(12)$ & $2.70(33)$ & 74(7) & $0.72(23)$ & $11^{3}$ & $0.06(2)$ \\
\hline 28475 & 20963 & 6.8 & B5 V & SB2 & 86 & 811 & 4.178 & 3.97 & $4.8(9)$ & $3.8(13)$ & $2.82(33)$ & $67(9)$ & $0.76(21)$ & $30^{5}$ & $0.15(5)$ \\
\hline 112396 & 63096 & 6.8 & A0 & & 197 & 1012 & 3.995 & 4.15 & $2.4(4)$ & $2.2(8)$ & $1.60(34)$ & $272(16)$ & $0.52(31)$ & & \\
\hline 138003 & 75641 & 7.9 & B9 & & 322 & 747 & 4.151 & 4.00 & $4.3(8)$ & $3.5(12)$ & $2.63(34)$ & 83(9) & $0.72(23)$ & & \\
\hline 179588 & 94377 & 6.7 & B9 IV & VB & 128 & 1151 & 4.085 & 4.28 & $3.1(4)$ & $2.2(5)$ & $1.99(24)$ & $94(37)$ & $0.34(28)$ & $35^{5}$ & $0.31(7)$ \\
\hline 182255 & 95260 & 5.2 & B6 III & SB1 & 176 & 769 & 4.149 & 4.23 & $3.9(6)$ & $2.6(7)$ & $2.39(28)$ & $58(18)$ & $0.39(31)$ & $14^{3}$ & $0.11(3)$ \\
\hline 191295 & 99250 & 7.2 & B7 III & & 166 & 467 & 4.118 & 4.14 & $3.6(6)$ & $2.7(9)$ & $2.29(33)$ & $91(9)$ & $0.51(31)$ & & \\
\hline 206540 & 107173 & 6.1 & B5 IV & & 170 & 1178 & 4.145 & 4.11 & $4.0(7)$ & $3.0(10)$ & $2.47(34)$ & $76(3)$ & $0.55(30)$ & $10^{3}$ & $0.07(2)$ \\
\hline 208057 & 108022 & 5.1 & B3 Ve & & 116 & 1162 & 4.221 & 4.15 & $5.1(8)$ & $3.2(10)$ & $2.85(31)$ & $34(9)$ & $0.46(33)$ & $110^{3}$ & $0.68(21)$ \\
\hline 222555 & 116870 & 7.4 & B9 & & 249 & 1151 & 4.122 & 4.19 & $3.6(6)$ & $2.6(8)$ & $2.26(31)$ & $78(21)$ & $0.43(32)$ & & \\
\hline 46005 & 31065 & 7.9 & $\mathrm{~B} 8 \mathrm{~V}$ & VB & 164 & 379 & 4.325 & 4.43 & $7.0(7)$ & $3.2(3)$ & $3.26(15)$ & $4(2)$ & $0.10(7)$ & & \\
\hline 154689 & 83467 & 8.2 & B9 & & 113 & 127 & 4.020 & 4.22 & $2.5(4)$ & $2.1(6)$ & $1.67(31)$ & 191(42) & $0.42(32)$ & & \\
\hline 169820 & 90337 & 6.4 & B9 V & & 124 & 147 & 4.071 & 4.26 & $2.9(4)$ & $2.2(6)$ & $1.93(26)$ & 112(39) & $0.37(29)$ & $136^{\circ}$ & $1.22(33)$ \\
\hline 208727 & 108348 & 6.5 & B8 V & & 290 & 1162 & 4.112 & 4.22 & $3.4(5)$ & $2.5(7)$ & $2.18(30)$ & $81(24)$ & $0.40(31)$ & $205^{5}$ & $1.62(45)$ \\
\hline
\end{tabular}

${ }^{1}$ Taken from the SIMBAD database, operated at CDS, Strasbourg, France; ${ }^{2}$ Obtained with the code CALIB (Künzli et al. 1997);

${ }^{3}$ Mathias et al. (2001) (upper values); ${ }^{4}$ Mokiem et al. (2005); ${ }^{5}$ Abt et al. (2002); ${ }^{6}$ Royer et al. (2002); ${ }^{7}$ Clark \& Steele (2000);

${ }^{8}$ Abt \& Morrell (1995); ${ }^{9}$ Hill (1967).

Observations in the GENEVA filters allow a physically meaningful classification of early-type stars. With the code CALIB, physical parameters can be determined for unreddened B to mid-G stars on the main sequence or just above it. It is based on the calibrations proposed by Künzli et al. (1997). For hot stars $\left(T_{\text {eff }}>11000 \mathrm{~K}\right)$, the reddening-free parameters:

$$
\begin{aligned}
X= & 0.3788+1.3764[U-B]-1.2162\left[B_{1}-B\right] \\
& -0.8498\left[B_{2}-B\right]-0.1554\left[V_{1}-B\right]+0.8450[G-B], \\
Y= & -0.8288+0.3235[U-B]-2.3228\left[B B_{1}-B\right] \\
& +2.3363\left[B_{2}-B\right]+0.7495\left[V_{1}-B\right]-1.0865[G-B],
\end{aligned}
$$

(defined by Cramer \& Maeder 1979) have the optimum efficiency for determining the effective temperature $T_{\text {eff }}$ and the surface gravity $\log (g)$, respectively. We used the mean GENEVA colours $[U-B],\left[B_{1}-B\right],\left[B_{2}-B\right],\left[V_{1}-B\right]$, and $[G-B]$ of observed O-B star to obtain $T_{\text {eff }}$ and $\log (g)$ by assuming a solar composition (Fig. 1). Since the errors provided by this code are interpolation errors, we prefer to use more realistic errors of 0.020 and $0.20 \operatorname{dex}$ on $\log \left(T_{\text {eff }}\right)$ and $\log (g)$, respectively (indicated by the cross in the bottom left corner of Fig. 1; Morel et al. 2006). The targets that are observed at more than 50 epochs are given with full symbols and the 28 objects for which the light curves are analysed in this paper are given in black. Their $\log \left(T_{\text {eff }}\right)$ and $\log (g)$ values are given in Cols. 8 and 9 of Table 1. If the code CALIB needed an extrapolation from the calibration tables, these values are given in italics. These results should be treated with caution, as well as those for the binaries, indicated in Col. 5 of Table 1. The position in the H-R diagram of some of the variable O-B stars with MERCATOR data already indicates that their classification at the start of our project in 2001, as shown in Fig. 1, cannot be correct.

For our analysis, we only retained the high-quality data with a typical precision of $5 \mathrm{mmag}$. We calculated the mean $\mu^{X}$ and the standard deviation $\sigma^{X}$ in each passband $X \in$ $\left\{U, B_{1}, B, B_{2}, V_{1}, V, G\right\}$ and additionally removed data points deviating more than $3 \sigma^{X}$ from $\mu^{X}$ (after having checked that we are not dealing with eclipses). The number of removed observations is generally much less than $10 \%$ of the number of retained ones, except for our brightest targets. Indeed, the photometry is less accurate for objects with $m_{V} \leq 5.5$. In the case of HD 214680 and HD 217782, we removed all the data from 7 nights because several observations within each of these nights showed values deviating much more than $3 \sigma^{X}$ from $\mu^{X}$ for both objects. For 
each target, the final number $N$ of high-quality observations and the corresponding total time span $\triangle T$ in days are given in Cols. 6 and 7 of Table 1 .

\section{Data analysis methods}

\subsection{Frequency analysis}

We performed time series analysis in all seven GENEVA passbands by comparing the results for the frequency interval $[0,15] \mathrm{d}^{-1}$ with a frequency step of $0.0001 \mathrm{~d}^{-1}$ found with two independent frequency search algorithms, i.e. phase dispersion minimisation (Stellingwerf 1978) and the modified SCARGLE periodogram (Horne \& Baliunas 1986). In the text below, we restrict ourselves to a discussion of the modified SCARGLE periodograms. If a frequency could be identified, we determined in each GENEVA passband the frequency that optimises the variance reduction. The amplitudes of the resulting frequencies were used to fix the frequency to the weighted mean $f_{j}$. Prewhitening was done by fitting its amplitude and phase, and additional frequencies were searched for in the residuals. An underestimate of the accuracy of the frequencies was computed for each filter as:

$\sigma_{f_{j}}^{X}=\sqrt{6} \sigma_{\text {res }}^{X} / \pi \sqrt{N} A_{j}^{X} \Delta T$

with $N$ the number of observations, $\Delta T$ their total time span, $A_{j}^{X}$ the amplitude of frequency $f_{j}$ and $\sigma_{\text {res }}^{X}$ the standard deviation of the final residuals in filter $X$ (cf. Montgomery \& O'Donoghue 1999). We adopted $\sigma_{f_{j}}=\sum_{X=U}^{G} \sigma_{f_{j}}^{X} / 7$ as a final estimate of the frequency accuracy. We only retained frequencies with an amplitude significance $\geq 3.6 \mathrm{~S} / \mathrm{N}$-level, where the $\mathrm{S} / \mathrm{N}$-level was computed as the running average amplitude over a frequency interval with a width of $2 \mathrm{~d}^{-1}$ in a modified SCARGLE periodogram obtained after final prewhitening. This procedure is appropriate for our kind of data sets, as shown by Alvarez et al. (1998) and De Cat \& Cuypers (2003). For O-B stars, the $U$-filter is best suited to detect pulsation frequencies since their amplitude is the highest in this filter. Therefore, we generally show the different steps of subsequent prewhitening in the $U$-filter in the left and middle panels of Figs. 2-29. The frequency analysis is stopped when none of the frequency peaks reaches the $3.6 \mathrm{~S} / \mathrm{N}$-level. The residual standard deviations $\sigma_{\text {res }}^{X}$ can be used to judge the possibility of having more intrinsic frequencies than the ones retained from our analysis.

Afterwards, we applied a final fit to the light curves in each GENEVA passband $X$ with all the $N_{f}$ accepted frequencies $f_{j}$ by using a superposition of sinusoidal models:

$y^{X}(t)=C^{X}+\sum_{j=1}^{N_{f}} A_{j}^{X} \sin \left[2 \pi\left(f_{j} t+\phi_{j}^{X}\right)\right]$,

where $t=\mathrm{HJD}-2450000$. The values and the corresponding standard errors of $f_{j}$, the amplitudes $A_{j}^{X}$, the phases $\phi_{j}^{X}$, the constant terms $C^{X}$, and the residual standard deviations $\sigma_{\text {res }}^{X}$ are given in Table 2. A similar frequency analysis procedure was applied to the observations in the HIPPARCos $H_{\mathrm{p}}$ filter. These results are given in Table 3.

\subsection{Mode identification}

For all the $N_{f}$ accepted frequencies $f_{j}$, the amplitudes $A_{j}^{X}$ were used to calculate the observed amplitude ratios $A_{j}^{X} / A_{j}^{U}$ to apply the method of photometric amplitudes, using the formalism of Dupret et al. (2003). This allows the determination of the most probable values for the degree $\ell_{j}$ of the pulsation mode responsible for the observed variations with frequency $f_{j}$. We followed the same photometric identification procedure as De Cat et al. (2005), but we used a different grid of equilibrium models. Our grid consists of main sequence models with mass $M$ between 2 and $15 M_{\odot}$ with steps of $0.1 M_{\odot}$ calculated with a recent version of the stellar evolution code CLÉS (version 18.2; written by R. Scuflaire) using the CEFF equation of state (ChristensenDalsgaard \& Daeppen 1992) and a Kurucz atmosphere with the junction point at optical depth $\tau=10$. The upper limit of $15 M_{\odot}$ is due to the lack of Kurucz atmosphere models for more massive stars. We assumed neither convective overshooting nor diffusion. The main improvement of CLÉS is the use of a new value of the cross section of ${ }^{14} \mathrm{~N}(p, \gamma){ }^{15} \mathrm{O}$ recently measured by Formicola et al. (2004). We decided to use the 'new' solar values, i.e. an initial mass fraction of metals $Z_{0}=0.015$ and of hydrogen $X_{0}=$ 0.71 , a mixing-length $\alpha_{\text {conv }}=1.75$, and the standard metal mixture of Asplund et al. (2005). As shown by De Cat et al. (2004a), the lower value of $Z_{0}$ helps to solve the instability problems observed for some of the SPBs. Moreover, this value agrees better with the recent metallicity determinations of Niemczura (2003).

For all the equilibrium models in our grid, we used the nonadiabatic pulsation code MAD (version 1.0; written by M.-A. Dupret) to check the instability of modes with frequency between 0.25 and $25 \mathrm{~d}^{-1}$ and to calculate their theoretical amplitude ratios. We considered $\ell \leq 4$ for both $p$ - and $g$-modes. Pulsation modes with higher $\ell$ are unlikely to be observed in photometry because of cancellation effects. For each star, we retained the models within the observed error box of $\log \left(T_{\text {eff }}\right)$ and $\log (g)$ (cf. Table 1 and Fig. 1), and selected for each $\ell$ value the eigenfrequency that is the closest to the observed frequency to compare the observed and theoretical amplitude ratios. For each $\ell$ value, we used the percentage of the selected models for which all the corresponding theoretical amplitude ratios are compatible with the observed ones as a probability indicator for the identification. The results are given in Table 4 and the right panels of Figs. 2-29. As can be seen in Fig. 1, a significant part of the observed $\left(\log \left(T_{\text {eff }}\right), \log (g)\right)$ error box falls outside the grid for some stars. In these cases, the results of the mode identification should be treated with caution. If the full error box falls outside the grid, we were unable to perform mode identification.

\subsection{Fundamental parameters}

For each star, we calculated the mean of the mass $M\left(M_{\odot}\right)$, the radius $R\left(R_{\odot}\right)$, the luminosity $\log \left(L / L_{\odot}\right)$ (dex), the age (expressed in $10^{6}$ years), and the fraction of the main sequence lifetime frac $_{\mathrm{MS}}$ for the same models that we used for the mode identification. The resulting values are given in Cols. 10-14 of Table 1, respectively. We scanned the literature to search for values of the projected rotation velocity $v_{\text {eq }} \sin i$. The most recent value (if available) is given in Col. 15 of Table 1. These values were used in combination with the radius to calculate the projected rotation frequency $f_{\Omega} \sin i=v_{\text {eq }} \sin i / 2 \pi R\left(\mathrm{~d}^{-1}\right)$, given in Col. 16 of Table 1. Because $f_{\Omega} \sin i$ corresponds to a lower limit of the rotation frequency $f_{\Omega}$, this value can be used to judge whether an observed frequency can be the rotation frequency, or if close observed frequencies can be components of one rotationally split mode.

\section{Results for individual stars}

This section, including Figs. 2-29, is available only in electronic form at http: //www . aanda.org. 
Table 4. Overview of the observed modes (frequencies and $\ell$ values) in the SPB stars (top), the $\beta$ Cep stars (middle) and the hybrid $\beta$ Cep/SPB stars (bottom). The frequencies needing further observational confirmation are given in italics. The $\ell$ values are sorted according to a decreasing probability from left to right. The $\ell$ values for which the theoretical amplitude ratios are compatible with the observed ones for all the considered models within the $\left(T_{\text {eff }}, \log (g)\right)$ error box are given in bold. Within each category, the objects are given according to a decreasing number of frequencies and/or identified modes. For the 7 objects for which the classification as a confirmed pulsator is new, the previous classification is indicated between brackets.

\begin{tabular}{|c|c|c|c|c|c|c|c|c|c|c|c|c|}
\hline $\begin{array}{l}\text { HDnr } \\
\text { classification }\end{array}$ & HIPnr & data & $\begin{array}{c}f_{1} \\
\left(\mathrm{~d}^{-1}\right)\end{array}$ & $\ell_{1}$ & $\begin{array}{c}f_{2} \\
\left(d^{-1}\right)\end{array}$ & $\ell_{2}$ & $\begin{array}{c}f_{3} \\
\left(d^{-1}\right)\end{array}$ & $\ell_{3}$ & $\begin{array}{c}f_{4} \\
\left(d^{-1}\right)\end{array}$ & $\ell_{4}$ & $\begin{array}{c}f_{5} \\
\left(d^{-1}\right)\end{array}$ & $\ell_{5}$ \\
\hline HD 182255 & HIP 95260 & $\begin{array}{l}\text { MERCATOR } \\
\text { HIPPARCOS }\end{array}$ & $\begin{array}{l}0.97185(3) \\
0.79214(3)\end{array}$ & 1,4 & $\begin{array}{l}0.79225(4) \\
0.97187(4)\end{array}$ & $\mathbf{1}, 4$ & $\begin{array}{l}0.62526(7) \\
0.62512(10)\end{array}$ & $1,4,2$ & $1.12780(10)$ & 1,4 & $1.02884(11)$ & $1,4,2$ \\
\hline HD 21071 & HIP 15988 & $\begin{array}{l}\text { MERCATOR } \\
\text { HIPPARCOS }\end{array}$ & $\begin{array}{l}1.18843(1) \\
1.18843(3)\end{array}$ & 1 & $\begin{array}{l}1.14934(3) \\
1.14942(6)\end{array}$ & $1,4,2$ & $1.41968(7)$ & 1,2 & $0.95706(9)$ & $\mathbf{1}, 4,2$ & & \\
\hline HD 179588 & HIP 94377 & $\begin{array}{l}\text { MERCATOR } \\
\text { HIPPARCOS }\end{array}$ & $\begin{array}{l}0.85654(4) \\
0.81599(7)\end{array}$ & $\mathbf{1}, 2,4$ & $2.04263(5)$ & & $2.19989(7)$ & $\mathbf{1}, 4,2,3$ & $1.83359(9)$ & $\mathbf{1}, 4,2,3$ & & \\
\hline HD 222555 & HIP 116870 & $\begin{array}{l}\text { MERCATOR } \\
\text { HIPPARCOS }\end{array}$ & $\begin{array}{l}0.56397(3) \\
0.56255(6)\end{array}$ & $\mathbf{1}, 2,4$ & $0.38543(7)$ & $1,4,2$ & $0.90754(6)$ & & $0.46435(10)$ & 2,1 & & \\
\hline HD 206540 & HIP 107173 & $\begin{array}{l}\text { MERCATOR } \\
\text { HIPPARCOS }\end{array}$ & $\begin{array}{l}0.72002(4) \\
0.71989(7)\end{array}$ & $\mathbf{1}, 4$ & $\begin{array}{l}0.62125(5) \\
0.62159(8)\end{array}$ & 2,4 & $0.38271(8)$ & $1,4,2$ & & & & \\
\hline HD 191295 & HIP 99250 & $\begin{array}{l}\text { MERCATOR } \\
\text { HIPPARCOS }\end{array}$ & $\begin{array}{l}0.71908(6) \\
0.71908(5)\end{array}$ & 1,4 & $0.49011(12)$ & $\mathbf{1}, 2,4$ & $0.29302(17)$ & $1,4,2$ & & & & \\
\hline HD 25558 & HIP 18957 & $\begin{array}{l}\text { MERCATOR } \\
\text { HIPPARCOS }\end{array}$ & $\begin{array}{l}0.65265(2) \\
0.65284(5)\end{array}$ & 1,4 & $\begin{array}{l}1.93235(8) \\
1.92977(22)\end{array}$ & $1,2,3,4$ & $1.17913(10)$ & $\mathbf{1}, 2,4,3$ & & & & \\
\hline HD 208057 & HIP 108022 & $\begin{array}{l}\text { MERCATOR } \\
\text { HIPPARCOS }\end{array}$ & $\begin{array}{l}0.89050(7) \\
0.80184(5)\end{array}$ & $\mathbf{1}, 4,2$ & $\begin{array}{l}0.80213(10) \\
0.89045(7)\end{array}$ & $1,2,4$ & $2.47585(8)$ & $2,3,4$ & & & & \\
\hline HD 1976 & HIP 1921 & $\begin{array}{l}\text { MERCATOR } \\
\text { HIPPARCOS }\end{array}$ & $\begin{array}{l}0.39946(4) \\
0.93914(3)\end{array}$ & & $\begin{array}{l}0.93895(4) \\
0.39934(5)\end{array}$ & $\mathbf{1}, 2,3,4$ & $1.20346(6)$ & $\mathbf{1}, 2,3,4$ & & & & \\
\hline HD 28114 & HIP 20715 & $\begin{array}{l}\text { MERCATOR } \\
\text { HIPPARCOS }\end{array}$ & $\begin{array}{l}0.48790(4) \\
0.79104(23)\end{array}$ & 1,4 & $0.49666(8)$ & 1,4 & & & & & & \\
\hline HD 3379 & HIP 2903 & MERCATOR & $1.82023(19)$ & $2,3,4$ & $1.59418(22)$ & $2,3,4$ & & & & & & \\
\hline HD 28475 & HIP 20715 & $\begin{array}{l}\text { MERCATOR } \\
\text { HIPPARCOS }\end{array}$ & $\begin{array}{l}0.68369(7) \\
0.68537(7)\end{array}$ & $2,3,4$ & $0.40893(13)$ & 1,4 & & & & & & \\
\hline $\begin{array}{l}\text { HD } 169820 \\
\text { (spotted star) }^{1}\end{array}$ & HIP 90337 & $\begin{array}{l}\text { MERCATOR } \\
\text { HIPPARCOS }\end{array}$ & $\begin{array}{l}2.12509(32) \\
2.12509(10)\end{array}$ & 4,2 & & & & & & & & \\
\hline HD 138003 & HIP 75641 & $\begin{array}{l}\text { MERCATOR } \\
\text { HIPPARCOS }\end{array}$ & $\begin{array}{l}0.66110(3) \\
0.66097(5)\end{array}$ & & & & & & & & & \\
\hline $\begin{array}{l}\text { HD } 14053 \\
\text { (rejected } \beta \text { Cep }\end{array}$ & & MERCATOR & $6.08608(11)$ & 4 & $9.61805(22)$ & 4,2 & $7.69877(21)$ & 4 & $2.74081(29)$ & 4 & $2.86425(80)$ & \\
\hline $\begin{array}{l}\text { HD } 89688 \\
\left(_{\text {constant })^{1}}\right.\end{array}$ & & MERCATOR & $0.79649(10)$ & $2,4,3$ & $5.79148(11)$ & 2,3 & $10.92814(10)$ & & $4.09715(16)$ & 0,1 & & \\
\hline $\begin{array}{l}\text { HD } 180968 \\
(\lambda \text { Eri star })^{3}\end{array}$ & HIP 94827 & $\begin{array}{l}\text { MERCATOR } \\
\text { HIPPARCOS }\end{array}$ & $\begin{array}{l}0.63754(8) \\
0.63747(7)\end{array}$ & $1,3,2$ & $2.18570(17)$ & $2,3,4$ & & & & & & \\
\hline $\begin{array}{l}\text { HD } 13831 \\
\text { (rejected } \beta \text { Cep }\end{array}$ & $\begin{array}{l}\text { HIP } 10615 \\
p)^{2}\end{array}$ & MERCATOR & $0.97525(8)$ & - & $5.41824(11)$ & - & & & & & & \\
\hline HD 214680 & HIP 111841 & MERCATOR & $3.2584(8)$ & - & & & & & & & & \\
\hline $\begin{array}{l}\text { HD } 13745 \\
(\text { constant })^{1}\end{array}$ & & $\begin{array}{l}\text { MERCATOR } \\
\text { HIPPARCOS }\end{array}$ & $\begin{array}{l}1.13358(6) \\
11.44972(6)\end{array}$ & - & $2.64441(6)$ & - & $0.68063(7)$ & - & $8.18548(11)$ & - & & \\
\hline $\begin{array}{l}\text { HD } 19374 \\
\text { (rejected } \beta \text { Cep }\end{array}$ & $\begin{array}{l}\text { HIP } 14514 \\
\text { p) }\end{array}$ & MERCATOR & $1.32663(4)$ & & $6.50027(9)$ & & $4.36573(12)$ & $1,2,3$ & & & & \\
\hline
\end{tabular}

${ }^{1}$ De Cat et al. (2004b); ${ }^{2}$ Stankov \& Handler (2005); ${ }^{3}$ Balona (1995).

\section{Summary and discussion}

We analysed the GENEVA (and HIPPARCOS) data of the 28 O-B stars with more than 50 high-quality observations collected during the first three years of scientific observations of the P7 photometer attached to the MERCATOR telescope. We contributed to the discussion of the existence of the Maia stars as a separate group of variables because four of our targets, i.e. HD 46005 , HD 154689, HD 169820, and HD 208727, were classified as such at the start of our project. HD 154689 is the only Maia candidate for which the effective temperature $T_{\text {eff }}$ and the surface gravity $\log (g)$, that we determined from the mean GENEVA colours, is consistent with this classification (rightmost star in Fig. 1). However, the Maia candidates are either reclassified as an SPB star (HD 169820) or we found indications in our MERCATOR data set that they show periodic variations due to external causes such as surface spots (HD 46005 and HD 154689) or binarity (HD 208727).

Of the 28 studied targets, we classified 21 as pulsating variables. An overview of the results of the frequency analysis and mode identification of the fourteen SPB stars, five $\beta$ Cep stars, 
and two hybrid $\beta \mathrm{Cep} / \mathrm{SPB}$ stars is given in the top, middle, and bottom rows of Table 4, respectively. For 7 of them, the classification as a confirmed pulsator is new. The Maia candidate HD 169820 is one of them. De Cat et al. (2004b) considered it as a candidate spotted star, but we now classified it as a rapidly rotating SPB star.

We detected a total of 61 frequencies in the MERCATOR data, of which at least $21(=35 \%)$ should be considered as wellestablished because they are also present in the HIPPARCOS $H_{\mathrm{p}}$ data and/or other data-sets. The other 40 frequencies need further observational confirmation. After 1.5 years of MERCATOR observations, De Cat et al. (2004b) found a total 27 frequencies for the 17 pulsating variables that are in common with our sample. After 3 years, the number of MERCATOR observations increased by $17 \%$ and we observed 50 frequencies for these stars (i.e. an increase of 85\%), of which 25 are new and 3 are aliases of the previously known frequencies. We could not confirm 2 of the frequencies reported by De Cat et al. (2004b). For HD 13831, HD 14053, HD 19374, and HD 214680, i.e. the four pulsating variables that were not analysed by De Cat et al. (2004b), only 3 of the 11 detected frequencies in the MERCATOR data could be identified with frequencies previously mentioned in the literature. For HD 182255, HD 208057, and HD 1976, the dominant frequency in the MERCATOR data corresponds to the second frequency in the HIPPARCOS data. The same scenario was found for the confirmed SPB star HD 215573 (De Cat \& Aerts 2002). For HD 179588, HD 28114, and HD 13745, none of the frequencies are commonly found in the MERCATOR and HIPPARCOS data, pointing to complex multi-periodic beating.

For six stars, we doubt that we are dealing with pulsating variables. We classified HD 24094, HD 112396, HD 149881, and the Maia candidate HD 208727 as ellipsoidal variables. Their dominant frequencies in the MERCATOR data, which correspond to $2 f_{\text {orb }}$, are $1.90105(2) \mathrm{d}^{-1}, 1.41491(4) \mathrm{d}^{-1}, 0.38445(4) \mathrm{d}^{-1}$ and $3.16780(1) \mathrm{d}^{-1}$, respectively (Table 2 ). Our classification is strengthened for HD 149881 and HD 24094 because they were already classified as a confirmed and suspected binary, respectively, and for HD 112396 because the available radial-velocity observations in the literature have a peak-to-peak amplitude of more than $100 \mathrm{~km} \mathrm{~s}^{-1}$. For the Maia candidates HD 46005 and HD 154689, we interpret the observed variations in terms of rotational modulation with $2.50476(2) \mathrm{d}^{-1}$ and $1.95767(110) \mathrm{d}^{-1}$, respectively, as the rotation frequency $f_{\Omega}$. For HD 154689, up to the second harmonic of $f_{\Omega}$ is observed, which is typical for spotted stars (North 1984; Briquet et al. 2004).

For the remaining star, i.e. the visual binary HD 217782, we were not able to detect periodic variations. In the periodogram, significant peaks occur only at frequencies invoked by the time sampling. We therefore classify it as photometrically constant up to better than 6 mmag. Our classification, especially for the nonpulsating stars, should be checked with (high-resolution) spectroscopic data. For the stars classified as spotted stars, we expect a different behaviour in the line-profiles of different chemical elements. For those we classified as binaries, we should observe large global Doppler shifts. Moreover, a radial-velocity curve is mandatory to enable accurate modelling of multiple systems.

For all the variable objects, except for HD 13831, HD 13745 , and HD 214680 because of the limitations of our grid, we interpreted the observed variations in terms of stellar pulsations by applying the method of photometric amplitude ratios. This is the first time that mode identification has been performed for the pulsating variables, resulting in 4 unambiguous identifications: one $\ell=1$ mode and three $\ell=4$ modes (Table 4 ). If we consider the most probable identifications, we end up with 1 ,
31,9 , and $5 \ell=0,1,2$, and 4 modes, respectively (leftmost $\ell$ value given for each mode in Table 4 ). In the case of equal probability, the lowest $\ell$ value was preferred because the cancellation decreases for decreasing $\ell$ values. The dominance of $\ell=1$ modes for the SPB stars is consistent with the recent results obtained with spectroscopic mode identifications (De Cat et al. 2005) and with theoretical predictions (Townsend 2003). The observed amplitude ratios with their $1 \sigma$ error are inconsistent with those of modes with $\ell \leq 4$ for 8 frequencies. The observed amplitude ratios of two of them, i.e. $f_{1}$ of HD 1976 and $f_{1}$ of HD 138003, resemble those of HD 53921, i.e. a close visual binary with components of a similar spectral type (De Cat et al. 2005). HD 1976 is also known as a close visual binary $(\rho=$ 0.'157(3), $\Delta m=0.89(10) \mathrm{mag}$ ), but HD 138003 is not known as such. For the other pulsating stars in our sample known to be components of a visual binary (HD 179588, HD 180968, and HD 214680), we did not observe the same kind of amplitude ratio behaviour.

For the non-pulsating variables, the application of the method of amplitude ratios was done only to check how well the behaviour of the amplitude ratios can be interpreted in terms of pulsation modes. For the binaries, the observed amplitude ratios are all close to 1 (Figs. 23-26). Those of HD 24094 and HD 208727 can be successfully identified with an $\ell=2$ (or 3 ) mode. This observation is not in contradiction with our classification as an ellipsoidal variable because a sectoral $\ell=2$ mode pulsating with $2 f_{\text {orb }}$ mimics an ellipsoid rotating with $f_{\text {orb }}$. Moreover, the higher the orbital frequency, the stronger the tidal forces, and the greater the deformation of the components. This gives a natural explanation for why the identification of the longer period binaries HD 112396 and HD 149881 fails. Also the variations with $f_{\Omega}$ of the spotted star HD 46005 could be successfully modelled with an $\ell=2$ mode, while the observed amplitude ratios for the dominant frequency $2 f_{\Omega}$ of HD 154689 are very atypical for stellar pulsation (Fig. 27).

For the multi-periodic SPB stars HD 1976, HD 3379, HD 21071, HD 25558, HD 28475, HD 179588, and HD 182255 , and for the multi-periodic $\beta$ Cep star HD 14053, some of the observed close frequencies may be components of the same rotationally split mode based on the value of the projected rotation frequency $f_{\Omega} \sin i$. However, the results of the mode identification do not always support such a scenario. For HD 28114, HD 182255, HD 191295, and HD 206540, we observed either close frequencies that differ by less than $1.5 / \triangle T$ (and hence the frequency values cannot be trusted; Loumos \& Deeming 1978) or frequencies that are almost exact aliases of each other. This possibly reflects that we are beginning to resolve (very) close frequency multiplets.

The detection of the new hybrid $\beta$ Cep/SPB stars HD 13745 and HD 19374 is extremely interesting from an asteroseismic point of view because they simultaneously pulsate in high-order $g$-modes and low-order $p / g$-modes, probing the deep interior and the outer layers, respectively. If our classification is correct, HD 13745 is the first hybrid $\beta$ Cep/SPB star in the hot common part of the theoretical instability strips of the $\beta$ Cep and SPB stars predicted by Pamyatnykh (1999). Before our study, the coexistence of short and long time-scale pulsations have been claimed for a few other hot stars: the well-known $\beta$ Cep star HD 29248 ( $v$ Eri: Jerzykiewicz et al. 2005, Handler et al. 2004) and the SPB stars HD 3379 (53 Psc: Le Contel et al. 2001), HD 160762 ( $\iota$ Her: Mathias \& Waelkens 1995; Chapellier et al. 2000), and HD 886 ( $\gamma$ Peg: Chapellier et al. 2006). We could not confirm this result for HD 3379 with the MERCATOR data. We also found simultaneous short and long time-scale variations for the Be stars 
HD 180968 and HD 13831, but in this case, we interpret the low frequency as the rotation frequency.

Acknowledgements. This research was made possible thanks to the financial support from the Fund for Scientific Research - Flanders (FWO), under projects G.0178.02 and G.0332.06, and from the Research Council of the University of Leuven under grant GOA/2003/04. K.U. acknowledges financial support from a European Union Marie Curie Intra-European Fellowship, under contract MEIFCT-2006-024476. We are indebted to our colleagues of the Geneva Observatory who reduced the MERCATOR data, and to our referee, Dr. Jaymie Matthews, for the valuable suggestions and remarks to improve this manuscript. This research has made use of the SIMBAD astronomical database operated at the CDS in Strasbourg, France.

\section{References}

Abt, H. A., \& Morrell, N. I. 1995, ApJS, 99, 135

Abt, H. A., \& Boonyarak, C. 2004, ApJ, 616, 562

Abt, H. A., Levato, H., \& Grosso, M. 2002, ApJ, 573, 359

Adelman, S. J. 2001, A\&A, 367, 297

Aerts, C., \& Kolenberg, K. 2005, A\&A, 431, 615

Aerts, C., Thoul, A., Daszyńska, J., et al. 2003, Science, 300, 1926

Aerts, C., de Cat, P., de Ridder, J., et al. 2006, A\&A, 449, 305

Alvarez, M., Hernandez, M. M., Michel, E., et al. 1998, A\&A, 340, 149

Andrews, K. E., \& Dukes, R. J. 2000, BAAS, 32, 1477

Andrews, J. E., Dukes, R. J., \& Mills, L. R. 2003, BAAS, 35, 1340

Asplund, M., Grevesse, N., \& Sauval, A. J. 2005, in Cosmic Abundances as

Records of Stellar Evolution and Nucleosynthesis, ASP Conf. Ser., 336, 25

Balona, L. A. 1995, MNRAS, 277, 1547

Balona, L. A., \& Marang, F. 1988, Informational Bulletin on Variable Stars, 3157, 1

Blaauw, A. 1956, ApJ, 123, 408

Bondal, K. R. 1967, The Observatory, 87, 220

Brandt, J. C., Heap, S. R., Beaver, E. A., et al. 1998, AJ, 116, 941

Briquet, M., Aerts, C., Lüftinger, T., et al. 2004, A\&A, 413, 273

Burki, G., Rufener, F., Burnet, M., et al. 1995, A\&AS, 112, 383

Chapellier, E., Le Contel, D., Le Contel, J. M., Mathias, P., \& Valtier, J.-C. 2006, A\&A, 448, 697

Chapellier, E., Mathias, P., Le Contel, J., et al. 2000, A\&A, 362, 189

Cheng, K.-P., \& Neff, J. E. 2003, AJ, 125, 868

Chochol, D., \& Grygar, J. 1976, Bulletin of the Astronomical Institutes of Czechoslovakia, 27, 181

Christensen-Dalsgaard, J., \& Daeppen, W. 1992, A\&ARv, 4, 267

Clark, J. S., \& Steele, I. A. 2000, A\&AS, 141, 65

Cramer, N., \& Maeder, A. 1979, A\&A, 78, 305

De Cat, P., \& Aerts, C. 2002, A\&A, 393, 965

De Cat, P., \& Cuypers, J. 2003, in Interplay of Periodic, Cyclic and Stochastic Variability in Selected Areas of the H-R Diagram, ASP Conf. Ser., 292, 377

De Cat, P., Daszyńska-Daszkiewicz, J., Briquet, M., et al. 2004a, in IAU Colloq. 193: Variable Stars in the Local Group, ASP Conf. Ser., 310, 195

De Cat, P., De Ridder, J., Uytterhoeven, K., et al. 2004b, in IAU Colloq. 193, Variable Stars in the Local Group, ASP Conf. Ser., 310, 238

De Cat, P., Briquet, M., Daszyńska-Daszkiewicz, J., et al. 2005, A\&A, 432, 1013

de Jager, C. 1953, Bull. Astr. Inst. Neth., 12, 91

De Ridder, J., Cuypers, J., De Cat, P., et al. 2004, in IAU Colloq. 193: Variable Stars in the Local Group, ASP Conf. Ser., 310, 263

Debosscher, J., Aerts, C., \& Vandenbussche, B. 2006, in Astrophysics of Variable Stars, ASP Conf. Ser., 349, 219

Delgado, A. J., \& Garrido, R. 1981, Informational Bulletin on Variable Stars, 1992, 1

Dukes, R. J., Kubinec, W. R., Kubinec, A., \& Adelman, S. J. 2003, AJ, 126, 370

Dupret, M. A. 2001, A\&A, 366, 166

Dupret, M.-A., De Ridder, J., De Cat, P., et al. 2003, A\&A, 398, 677

ESA. 1997, The Hipparcos and Tycho Catalogues (ESA SP-1200; Noordwijk: ESA)

Fehrenbach, C., Burnage, R., Duflot, M., et al. 1987, A\&AS, 71, 263

Formicola, A., Imbriani, G., Costantini, H., et al. 2004, Phys. Lett. B, 591, 61

Garrido, R., \& Delgado, A. J. 1982, Informational Bulletin on Variable Stars, 2080, 1

Gerbaldi, M., Faraggiana, R., \& Lai, O. 2003, A\&A, 412, 447

Gies, D. R. 1994, in Pulsation, Rotation, and Mass Loss in Early-Type Stars, IAU Symp., 162, 89
Gonzalez Bedolla, S. 1994, in Pulsation, Rotation, and Mass Loss in Early-Type Stars, IAU Symp., 162, 31

Grygar, J. 1963, Bull. Astr. Inst. Neth., 17, 305

Handler, G., Jerzykiewicz, M., Rodríguez, E., et al. 2006, MNRAS, 365, 327

Handler, G., Shobbrook, R. R., Jerzykiewicz, M., et al. 2004, MNRAS, 347, 454

Hill, G. 1967, ApJS, 14, 263

Hill, G., Odgers, G. J., \& Drolet, B. 1976, A\&A, 51, 1

Horne, J. H., \& Baliunas, S. L. 1986, ApJ, 302, 757

Hube, D. P. 1983, JRAS Can., 77, 142

Hube, D. P., \& Aikman, G. C. L. 1991, PASP, 103, 49

Humphreys, R. M. 1978, ApJS, 38, 309

Jerzykiewicz, M. 1974, PASP, 86, 43

Jerzykiewicz, M., \& Sterken, C. 1990, A\&A, 227, 77

Jerzykiewicz, M., Handler, G., Shobbrook, R. R., et al. 2005, MNRAS, 360, 619

Kallinger, T., Reegen, P., \& Weiss, W. W. 2002, A\&A, 388, L37

Kamper, K. W. 1974, AJ, 79, 54

Kaper, L., Henrichs, H. F., Nichols, J. S., \& Telting, J. H. 1999, A\&A, 344, 231

Koch, R. H. 1990, Bulletin d'Information du Centre de Donnees Stellaires, 38, 175

Koen, C., \& Eyer, L. 2002, MNRAS, 331, 45

Kohoutek, L., \& Wehmeyer, R. 1999, A\&AS, 134, 255

Künzli, M., North, P., Kurucz, R. L., \& Nicolet, B. 1997, A\&AS, 122, 51

Lane, M. C. 1976, Informational Bulletin on Variable Stars, 1202, 1

Le Contel, J. M., Chapellier, E., Valtier, J. C., et al. 1988, Informational Bulletin on Variable Stars, 3131, 1

Le Contel, J.-M., Mathias, P., Chapellier, E., \& Valtier, J.-C. 2001, A\&A, 380, 277

Loumos, G. L., \& Deeming, T. J. 1978, Ap\&SS, 56, 285

Lynds, C. R. 1959, ApJ, 130, 577

Mason, B. D., Gies, D. R., Hartkopf, W. I., et al. 1998, AJ, 115, 821

Mathews, R. T. 1956, PASP, 68, 455

Mathias, P., \& Waelkens, C. 1995, A\&A, 300, 200

Mathias, P., Aerts, C., Briquet, M., et al. 2001, A\&A, 379, 905

Mokiem, M. R., de Koter, A., Puls, J., et al. 2005, A\&A, 441, 711

Montgomery, M. H., \& O’Donoghue, D. 1999, Delta Scuti Newsletter, 13, 28

Morel, T., Butler, K., Aerts, C., Neiner, C., \& Briquet, M. 2006, A\&A, 457, 651

Münch, G., \& Flather, E. 1957, PASP, 69, 142

Murdoch, K. A., Drew, J. E., \& Anderson, L. S. 1994, A\&A, 284, L27

Niemczura, E. 2003, A\&A, 404, 689

North, P. 1984, A\&AS, 55, 259

Pamyatnykh, A. A. 1999, Acta Astron., 49, 119

Parenago, P. P. 1958, Sov. Astron., 2, 151

Percy, J. R. 1971, AJ, 76, 1105

Percy, J. R., \& Au-Yong, K. 2000, Informational Bulletin on Variable Stars, 4825, 1

Plasket, J. S., Harper, W. E., Young, R., \& Plaskett, H. 1921, Publ. Dominion Astrophys. Obs., 1, 300

Plasket, J. S., \& Pearce, J. A. 1930, Publ. Dominion Astrophys. Obs., 5, 1

Pribulla, T., Kreiner, J. M., \& Tremko, J. 2003, Contributions of the Astronomical Observatory Skalnate Pleso, 33, 38

Royer, F., Grenier, S., Baylac, M.-O., Gómez, A. E., \& Zorec, J. 2002, A\&A, 393,897

Rufener, F. 1986, A\&A, 165, 275

Sahade, J., Struve, O., \& Williams, A. D. 1956, PASP, 68, 266

Sareyan, J.-P., Le Contel, J.-M., Ducatel, D., \& Valtier, J.-C. 1979, A\&A, 72, 313

Scholz, G., Lehmann, H., Hildebrandt, G., Panov, K., \& Iliev, L. 1998, A\&A, 337,447

Smith, M. A. 1978, ApJ, 224, 927

Stagg, C. 1983, Informational Bulletin on Variable Stars, 2376, 1

Stankov, A., \& Handler, G. 2005, ApJS, 158, 193

Stellingwerf, R. F. 1978, ApJ, 224, 953

Sterken, C. 1988, A\&A, 189, 81

Struve, O. 1955, Sky \& Tel., 14, 461

Townsend, R. H. D. 2003, MNRAS, 343, 125

Underhill, A. B., \& van Helden, R. C. P. 1966, AJ, 71, 183

van den Bos, W. 1960, Publ. Yerkes Observatory, 9, 103

Van Hoof, A., \& Blaauw, A. 1964, Bull. Astr. Inst. Neth., 17, 451

Waelkens, C. 1991, A\&A, 246, 453

Waelkens, C., Aerts, C., Kestens, E., Grenon, M., \& Eyer, L. 1998, A\&A, 330, 215

Walker, M. F. 1952, AJ, 57, 227

Williams, A. D. 1954, PASP, 66, 88

Wolf, M. 1987, Informational Bulletin on Variable Stars, 3003, 1 
P. De Cat et al.: Long term photometric monitoring with the Mercator telescope, Online Material p 1

\section{Online Material}




\section{Results for individual stars}

In this section, we describe the results for the individual stars. These results allow us to (re)classify them as either an SPB star (Sect. 4.1.1), a $\beta$ Cep star (Sect. 4.1.2), a hybrid $\beta$ Cep/SPB star (Sect. 4.1.3), a binary (Sect. 4.2.1), a spotted star (Sect. 4.2.2), or a constant star (Sect. 4.3). In each subsection, the stars are sorted according to a decreasing number of detected frequencies and/or increasing HD number.

\subsection{Pulsating variables}

\subsubsection{Slowly pulsating B stars}

Dukes et al. (2003) studied HD 182255 (HIP 95260, HR 7358, $3 \mathrm{Vul}=\mathrm{V} 377 \mathrm{Vul})$ in detail on the basis of 7 seasons of data collected with the Four College Consortium Automatic Photoelectric Telescope (APT) and 17 coudé spectra obtained with the $1.22-\mathrm{m}$ telescope of the Dominion Astrophysical Observatory (DAO). They found three main frequencies $\left(0.9719 \mathrm{~d}^{-1}, 0.7923 \mathrm{~d}^{-1}, 0.8553 \mathrm{~d}^{-1}\right)$ and one combination frequency $\left(1.76420 \mathrm{~d}^{-1}\right)$ and could confirm the spectroscopic 367.26 orbit first determined by Hube \& Aikman (1991). Their first two main frequencies were previously observed in the AURÉLIE spectra of Mathias et al. (2001), who found, apart from $0.47233(3) \mathrm{d}^{-1}$, two additional possible frequencies in the HIPPARCOS data, leading to a suggestion of a frequency quintuplet. In the MERCATOR data, we find five frequencies (Fig. 2), of which two are known $\left(f_{1}=0.97185(3) \mathrm{d}^{-1}, f_{2}=0.79225(4) \mathrm{d}^{-1}\right)$ and three are new $\left(f_{3}=0.62526(7) \mathrm{d}^{-1}, f_{4}=1.12780(10) \mathrm{d}^{-1}\right.$, and $\left.f_{5}=1.02884(11) \mathrm{d}^{-1}\right)$. A re-analysis of the HIPPARCOS data leads to $f_{1}^{\mathrm{h}}=0.79214(3) \mathrm{d}^{-1}, f_{2}^{\mathrm{h}}=0.97187(4) \mathrm{d}^{-1}$, and $f_{3}^{\mathrm{h}}=$ $0.62512(10) \mathrm{d}^{-1}$. Contrary to Mathias et al. (2001), we retained $f_{3}^{\text {h }}$ because of the agreement with the MERCATOR data, although it is not the highest peak. Since there is no signal around $f_{4}$ or $f_{5}$ in the HIPPARCOS residuals, we are not sure about the real frequency values. Especially $f_{5}$ should be treated with caution, because $f_{1}+f_{5} \simeq 2.0 \mathrm{~d}^{-1}$, which might indicate that $f_{5}$ is an artefact or that we are beginning to resolve a close frequency multiplet. For all the modes of HD 182255, the best fit is found for $\ell=$ 1 although the identification is ambiguous (Table 4 and Fig. 2, right panels). For $\left(f_{2}, f_{1}, f_{4}\right)$ or $\left(f_{3}, f_{2}, f_{1}\right)$ to belong to a rotationally split $\ell=1$ multiplet, we need a rotation frequency $f_{\Omega}=$ $0.33(3) \mathrm{d}^{-1}$. This is compatible with the lower limit $f_{\Omega} \sin i=$ $0.11(3) \mathrm{d}^{-1}$ derived from the stellar parameters given in Table 1 and would imply $i<20^{\circ}$.

HD 21071 (HIP 15988, HR 1029, V576Per) is clearly a multi-periodic pulsator. The two main frequencies of the MERCATOR data, i.e. $f_{1}=1.18843(1) \mathrm{d}^{-1}$ and $f_{2}=1.14934(3) \mathrm{d}^{-1}$, coincide with those of the HIPPARCOS data (Table 4). These two frequencies were recently also observed in APT data by Andrews et al. (2003), who suggested the presence of additional periodicities. Indeed, in our MERCATOR data, we have evidence for two new frequencies: $f_{3}=1.41968(7) \mathrm{d}^{-1}$ and $f_{4}=0.95706(9) \mathrm{d}^{-1}$ (Fig. 3). However, due to the strong aliasing and the lack of agreement with the candidate frequencies in the HIPPARCOS data, we are not sure to have picked the correct ones. We unambiguously identify $f_{1}$ as $\ell=1$, while the observed variations of the other modes are also compatible with $\ell=2$ or 4 (Table 4 and Fig. 3, right panels). For $f_{1}$ and $f_{2}$ to be components of a rotationally split $\ell=1$ mode, $f_{\Omega}$ should be close to $0.078 \mathrm{~d}^{-1}$, which is much lower than the lower limit $f_{\Omega} \sin i=0.45(12) \mathrm{d}^{-1}$ (Table 1). However, the observed frequency spacing is compatible with the theoretical spacing of $\ell=1$ modes of subsequent radial orders, or between an $\ell=1$ and an $\ell=2$ or 4 mode in this frequency range for appropriate stellar models. On the other hand, $f_{\Omega} \sin i$ corresponds to the rotation frequency needed for $\left(f_{4}, f_{1}, f_{3}\right)$ to be a rotationally split $\ell=1$ mode.

For HD 179588 (HIP 94377, HR 7285, V338 Sge), there is evidence for the presence of at least four periodic signals in the MERCATOR light curves. Our frequency search points towards $f_{1}=0.85654(4) \mathrm{d}^{-1}, f_{2}=2.04263(5) \mathrm{d}^{-1}, f_{3}=2.19989(7) \mathrm{d}^{-1}$, and $f_{4}=1.83359(9) \mathrm{d}^{-1}$ (Fig. 4). None of these frequencies corresponds to the first frequency $f_{1}^{\mathrm{h}}=0.81599(7) \mathrm{d}^{-1}$ found in the HIPPARCos data. Due to the strong aliasing, we cannot rule out that we selected aliases of the true frequencies $\left(f_{2}\right.$ and $f_{3}$ are aliases of the frequencies retained by De Cat et al. 2004b). The results of the mode identification should be treated with caution because HD 179588 is a close visual binary for which the angular separation $\rho$ and magnitude difference $\Delta m$ between the two visual components were 0.' 664(2) and 0.83(1) mag, respectively, at the epoch of the HIPPARCOS observations (ESA 1997). We find $\ell=1$ as the most probable identification for $f_{1}, f_{3}$ and $f_{4}$, but the identification is ambiguous. The observed amplitude ratios of $f_{2}$ are compatible with none of the considered theoretical modes (Table 4 and Fig. 4, right panels). The close frequencies $f_{1}^{\mathrm{h}}$ and $f_{1}$ may be components of a rotationally split mode. Among the best candidates for the fifth frequency, we find $0.79467 \mathrm{~d}^{-1}$, but its modified SCARGLE amplitude does not exceed the 3.6 S/N-level. This frequency would fit in a split multiplet if we are dealing with an $\ell \geq 2$ mode, since $f_{1}-f_{1}^{\mathrm{h}} \simeq 2\left(f_{1}^{\mathrm{h}}\right.$ $0.79467)$. The mode identification for $f_{1}$ does not contradict such a scenario. However, the observed $v_{\text {eq }} \sin i$ value is not in favour of this possibility: the observed frequency spacings can only be explained if $f_{\Omega}$ is close to $0.025 \mathrm{~d}^{-1}$, but this value is much lower than $f_{\Omega} \sin i=0.31(7) \mathrm{d}^{-1}$ (Table 1). On the other hand, $f_{\Omega} \sin i$ is close to the rotation frequency needed for $f_{2}, f_{3}$, and/or $f_{4}$ to be members of a rotationally split mode.

Before the launch of the HIPPARCOS mission, HD 222555 (HIP 116870, V394 And) was not known as a variable. The main frequency in the MERCATOR data, $f_{1}=0.56397(3) \mathrm{d}^{-1}$, is very close to the main frequency in the HIPPARCOS data (Table 4). In the MERCATOR data, there is marginal evidence for at least three additional frequencies in the GENEVA $U$ passband: $f_{2}=0.38543(7) \mathrm{d}^{-1}$ and $f_{3}=0.90754(6) \mathrm{d}^{-1}$, and $f_{4}=$ $0.46435(10) \mathrm{d}^{-1}$ (Fig. 5). Also $1.81279 \mathrm{~d}^{-1}$ is a good candidate for an additional frequency, but it does not reach the $3.6 \mathrm{~S} / \mathrm{N}$ level. The additional frequencies should be considered as candidates only because they are found in a different order in the other GENEVA passbands, they do not exceed the $3.6 \mathrm{~S} / \mathrm{N}$-level in all the passbands (e.g. $f_{3}$ in the $U$-filter, see Fig. 5), and there is no agreement with the variations in the residual HIPPARCOS data, where $0.69445 \mathrm{~d}^{-1}$ is the best candidate for a second frequency. In any case, we classify HD 222555 as a multi-periodic SPB. Mode identification leads to $\ell=1$ as the best overall solution for $f_{1}$ and $f_{2}$, and to $\ell=2$ for $f_{4}$, while the observed amplitude ratios of $f_{3}$ are not compatible with any of the considered theoretical modes with $\ell \leq 4$ (Table 4 and Fig. 5, right panels). Since no $v_{\text {eq }} \sin i$ value is available for HD 222555, we cannot judge upon the possibility of frequencies being components of a rotationally split mode.

Mathias et al. (2001) included HD 206540 (HIP 107173, HR 8292) in their targetlist of northern suspected SPBs for spectroscopic monitoring. They found this star to be multi-periodic, but they were unable to separate the physical frequencies from the aliases. We now have evidence that their best candidates for the main HIPPARCOS frequency, i.e. $0.65359(4) \mathrm{d}^{-1}$ and $0.76237(4) \mathrm{d}^{-1}$, do not correspond to the physical one. Indeed, 


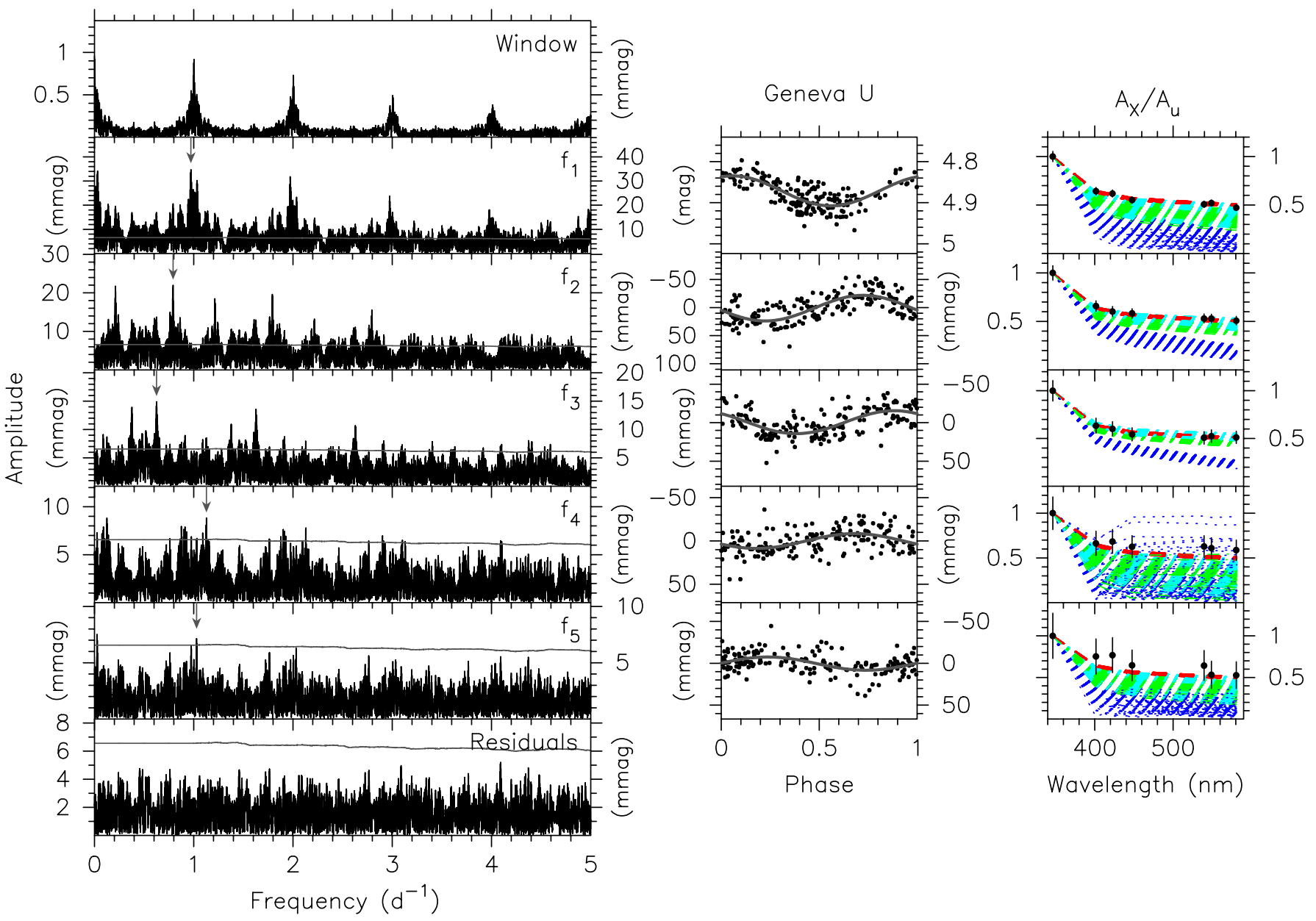

Fig. 2. Results for HD 182255. In the left panels, we show the window function (top), and the modified SCARGLE periodograms in the different steps of subsequent prewhitening in the GENEVA $U$ passband. The detected frequencies $f_{1}=0.97185(3) \mathrm{d}^{-1}, f_{2}=0.79225(4) \mathrm{d}^{-1}, f_{3}=0.62526(7) \mathrm{d}^{-1}$, $f_{4}=1.12780(10) \mathrm{d}^{-1}$, and $f_{5}=1.02884(11) \mathrm{d}^{-1}$ are indicated with arrows. The grey line corresponds to the $3.6 \mathrm{~S} / \mathrm{N}$-level. In the middle panels, we show phase diagrams with the detected frequencies. The dots and the grey full line denote the observations and the harmonic fit, respectively. The right panels show the results of the photometric mode identification. For each theoretical model within the observed $\operatorname{range}$ of $\log \left(T_{\text {eff }}\right) \operatorname{and} \log (g)$ (cf. Fig. 1 and Table 1), the theoretical amplitude ratios for modes with $\ell=1,2,3$, and 4 are represented with a red dashed, a green dash-dotted, a blue dotted, and a cyan dash-dot-dot-dotted line, respectively. The dots indicate the observed amplitude ratios and their standard error.

our MERCATOR data point towards $f_{1}=0.72002(4) \mathrm{d}^{-1}$ (Fig. 6) as the main frequency for HD 206540. This frequency corresponds to $f_{1}^{\mathrm{h}}=0.71989(7) \mathrm{d}^{-1}$, which is the fourth highest peak in the modified SCARGLE periodogram resulting from the HIPPARCOS $H_{\mathrm{p}}$ data. Moreover, the frequency differences between $f_{1}^{\mathrm{h}}$ and the best candidates given by Mathias et al. (2001) correspond to the highest peaks in the window function of the HIPPARCOS data. We therefore accept $f_{1}^{\mathrm{h}}$ as the main HIPPARCOS frequency for HD 206540. After prewhitening, we find $f_{2}=0.62125(5) \mathrm{d}^{-1}$ and $f_{3}=0.38271(8) \mathrm{d}^{-1}$ in the MERCATOR data (Fig. 6), of which $f_{2}$ corresponds to the second frequency in the HIPPARCos data (Table 4). The results of the mode identification are ambiguous. The most probable identifications for $f_{1}, f_{2}$, and $f_{3}$ are $\ell=1,2$, and 1 , respectively (Table 4 and Fig. 6 , right panels). Note that $f_{3}$ is close to $1-f_{2}$, and that there is evidence for an alias frequency of $f_{1}$ in the residuals, i.e. $2.72274 \mathrm{~d}^{-1}$. This may indicate that $f_{2}$ and/or $f_{3}$ are/is not an intrinsic frequency, but that we begin to resolve a close frequency multiplet for this slow rotator $\left(v_{\mathrm{eq}} \sin i<10 \mathrm{~km} \mathrm{~s}^{-1}\right.$; Mathias et al. 2001).

Waelkens et al. (1998) first classified HD 191295 (HIP 99250, V1473 Aql) as a new suspected SPB on the basis of the observed variations in the HIPPARCOS data. The first frequency in the MERCATOR data, i.e. $f_{1}=0.71908(6) \mathrm{d}^{-1}$, agrees perfectly with the one in the HIPPARCOS data (Table 4). After prewhitening, there are no significant peaks left in the HIPPARCOS periodogram. For the prewhitened MERCATOR data, there are several significant peaks which are each other's alias, of which $f_{2}=0.49011(12) \mathrm{d}^{-1}$ and $0.51271(17) \mathrm{d}^{-1}$ are the highest. To check the presence of even more frequencies, we additionally prewhitened with $f_{2}$. In the resulting periodograms, there are still significant peaks for $f_{3}=0.29302(29) \mathrm{d}^{-1}$ and its aliases. After prewhitening with $f_{3}$, there are no longer any significant peaks (Fig. 7, bottom panel). We therefore conclude that HD 191295 is a multi-periodic SPB, but, apart from the main frequency, we are not yet able to derive the frequency values. The photometric identification shows that observed variations are most probably invoked by $\ell=1$ modes, even though the identifications are ambiguous (Table 4 and Fig. 7 , right panels). As for HD 206540, $f_{3}$ is close to $1-f_{1}$. However, since an $v_{\text {eq }} \sin i$ value is not available, we cannot estimate the spacings expected for rotationally split frequency multiplets.

For HD 25558 (HIP 18957, HR 1253, V1133 Tau), there is evidence for up to three frequencies in the MERCATOR data: $f_{1}=$ $0.65265(2) \mathrm{d}^{-1}, f_{2}=1.93235(8) \mathrm{d}^{-1}$, and $f_{3}=1.17913(10) \mathrm{d}^{-1}$ or one of their aliases (Fig. 8). The first two are close to 
P. De Cat et al.: Long term photometric monitoring with the Mercator telescope, Online Material p 4

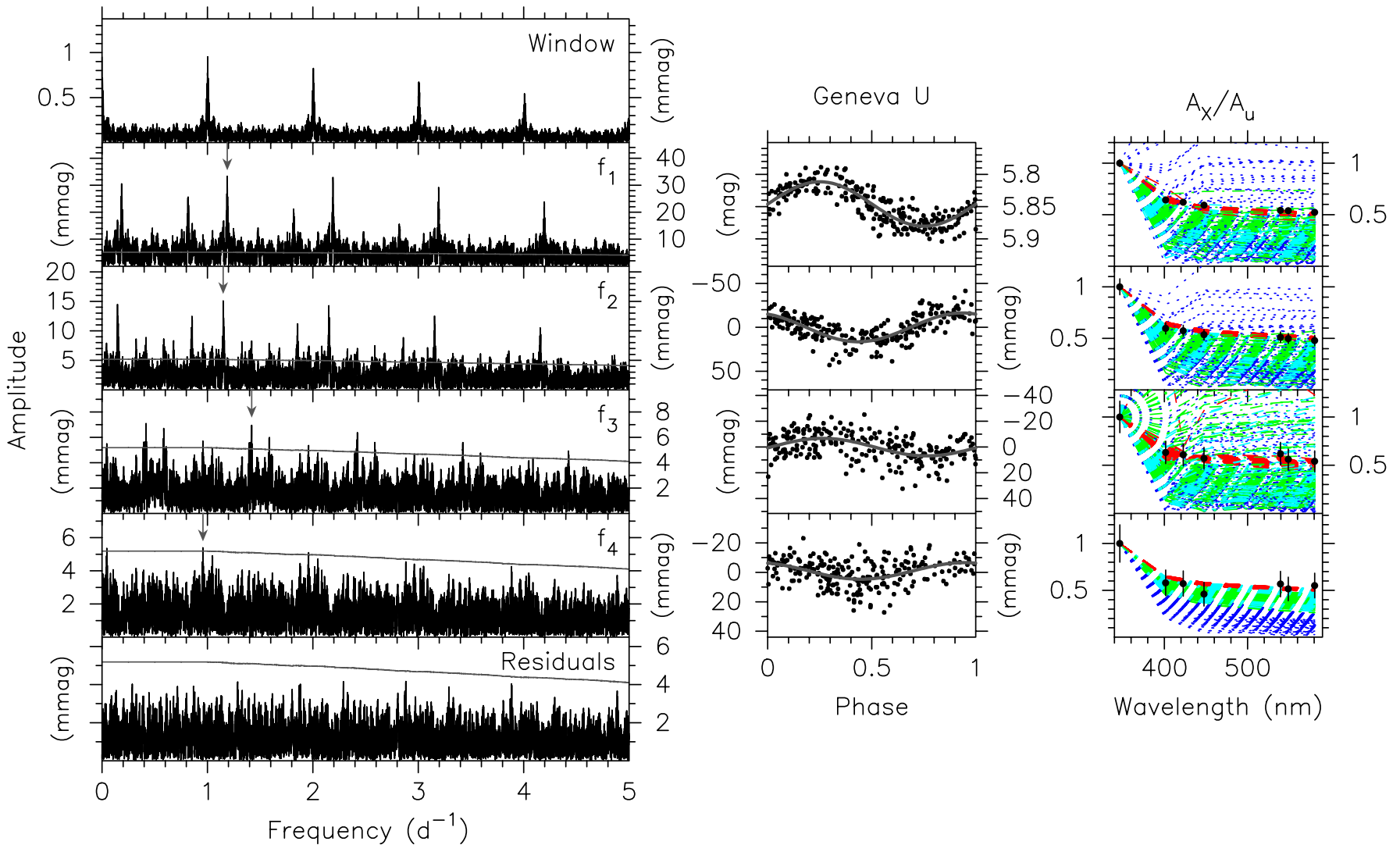

Fig. 3. Same as Fig. 2, but for $f_{1}=1.18843(1) \mathrm{d}^{-1}, f_{2}=1.14934(3) \mathrm{d}^{-1}, f_{3}=1.41968(7) \mathrm{d}^{-1}$, and $f_{4}=0.95706(9) \mathrm{d}^{-1}$ in the GENEVA $U$ passband of HD 21071.

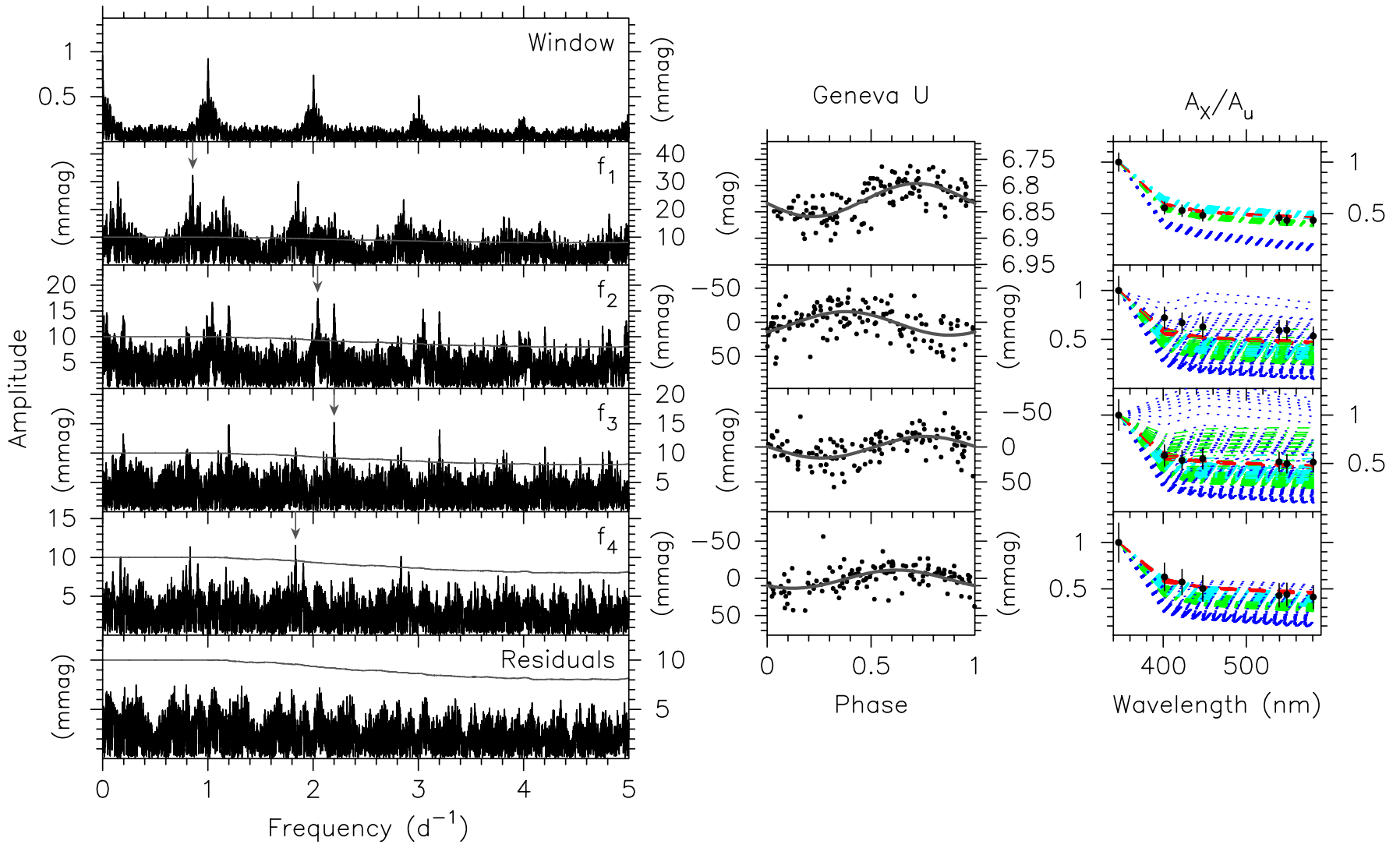

Fig. 4. Same as Fig. 2, but for $f_{1}=0.85654(4) \mathrm{d}^{-1}, f_{2}=2.04263(5) \mathrm{d}^{-1}, f_{3}=2.19989(7) \mathrm{d}^{-1}$, and $f_{4}=1.83359(9) \mathrm{d}^{-1}$ in the GENEVA $U$ passband of HD 179588. 
P. De Cat et al.: Long term photometric monitoring with the Mercator telescope, Online Material p 5
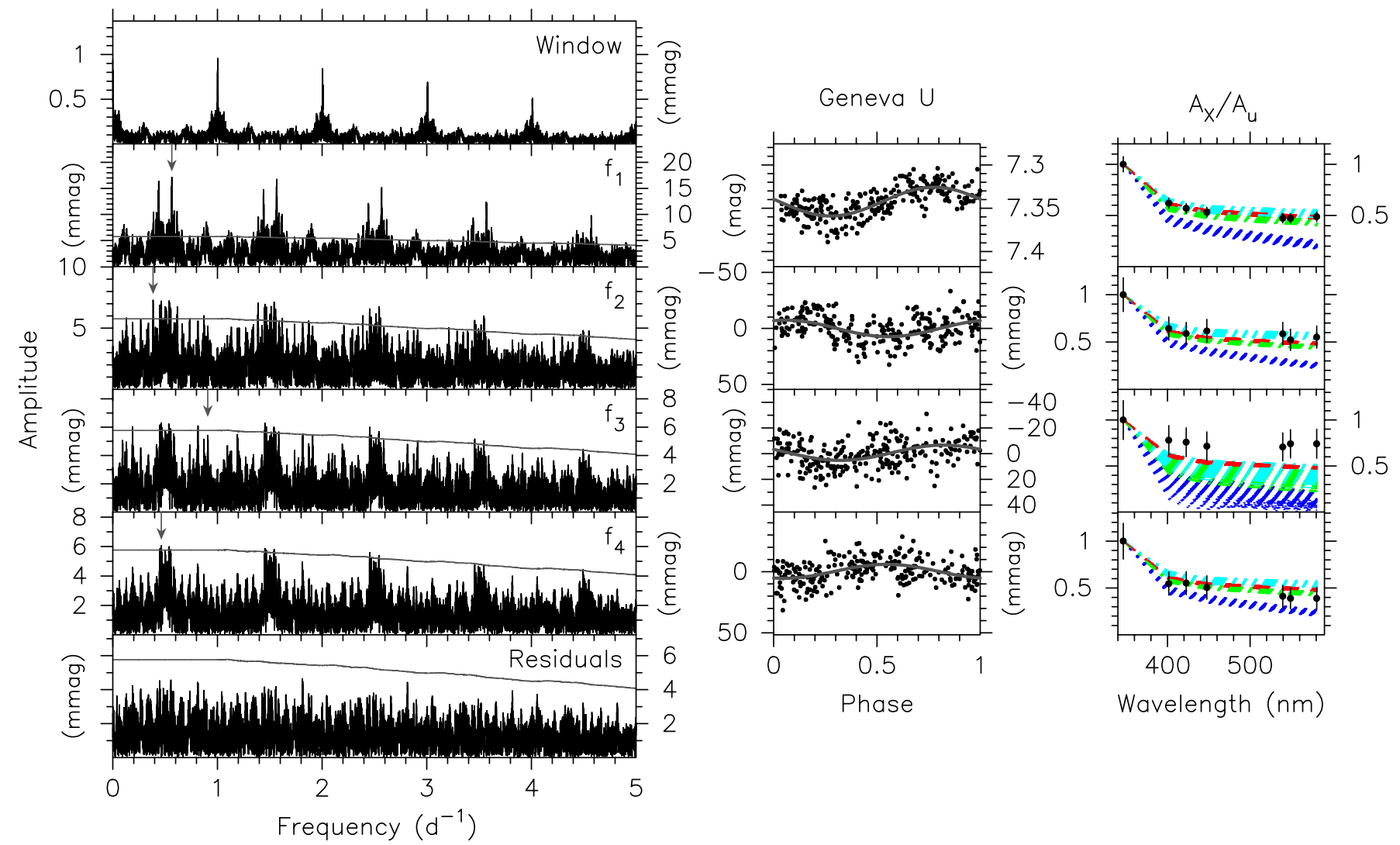

Fig. 5. Same as Fig. 2, but for $f_{1}=0.56397(3) \mathrm{d}^{-1}, f_{2}=0.38543(7) \mathrm{d}^{-1}, f_{3}=0.90754(6) \mathrm{d}^{-1}$, and $f_{4}=0.46435(10) \mathrm{d}^{-1}$ in the GENEVA $U$ passband of $\mathrm{HD} 222555$. The $3.6 \mathrm{~S} / \mathrm{N}$-level is not reached for $f_{3}$ in this passband.
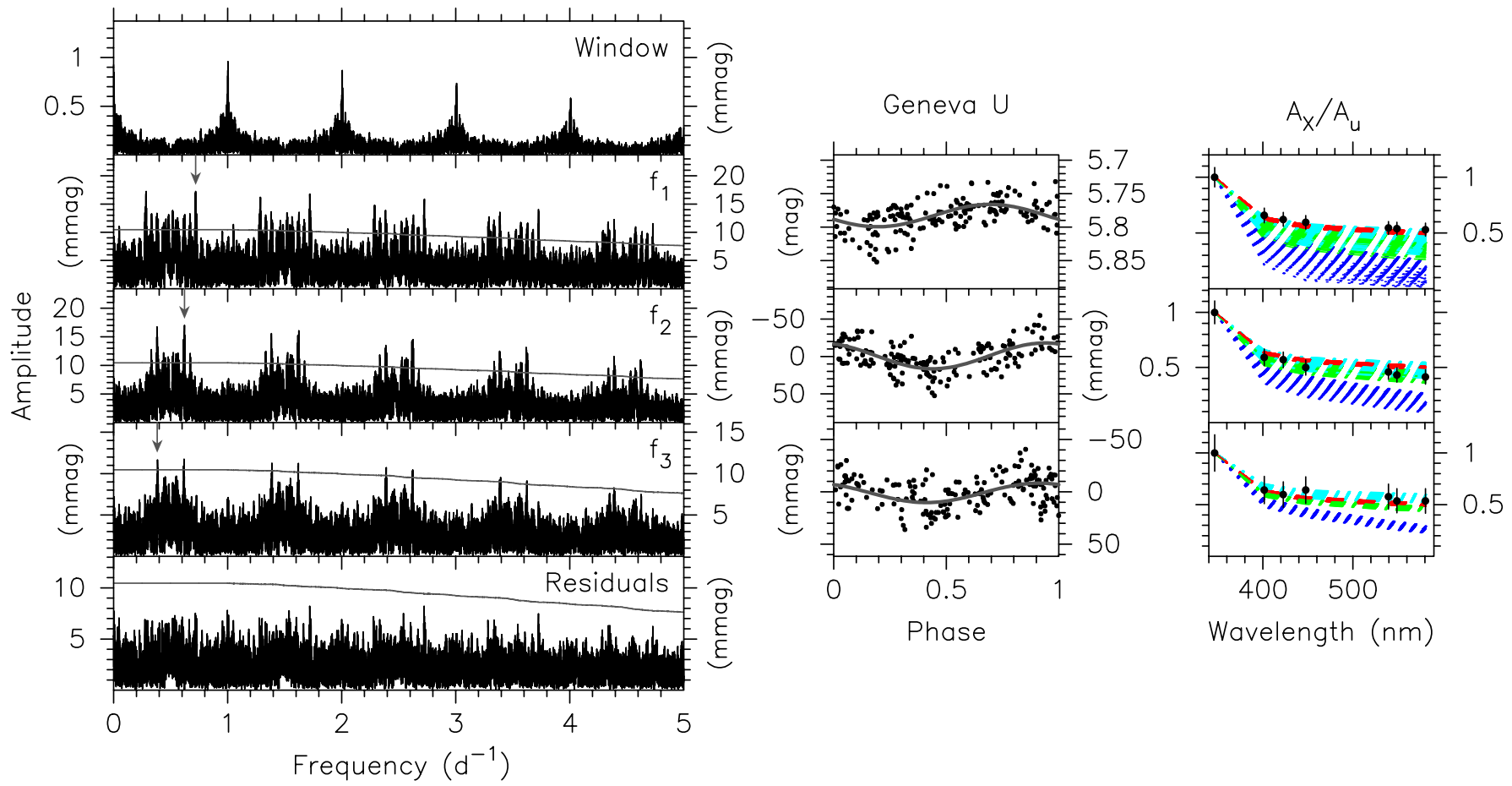

Fig. 6. Same as Fig. 2, but for $f_{1}=0.72002(4) \mathrm{d}^{-1}, f_{2}=0.62125(5) \mathrm{d}^{-1}$, and $f_{3}=0.38271(8) \mathrm{d}^{-1}$ in the GENEVA $U$ passband of HD 206540 .

$f_{1}^{\mathrm{h}}=0.65284(5) \mathrm{d}^{-1}$ and $f_{2}^{\mathrm{h}}=1.92977(22) \mathrm{d}^{-1}$ as found in the HIPPARCOS data. Note that $f_{2}$ does not correspond to the highest peak in the modified SCARGLE periodogram, but this frequency was preferred due to the similarity with $f_{2}^{\mathrm{h}}$, which was one of the two candidate frequencies in the HIPPARCos data proposed by Mathias et al. (2001). The mode identification is ambiguous for all modes, but the best fit is found for $\ell=1$ (Table 4 and Fig. 8, right panels). Given that $f_{\Omega} \sin i=0.14(4) \mathrm{d}^{-1}$ (Table 1), a 

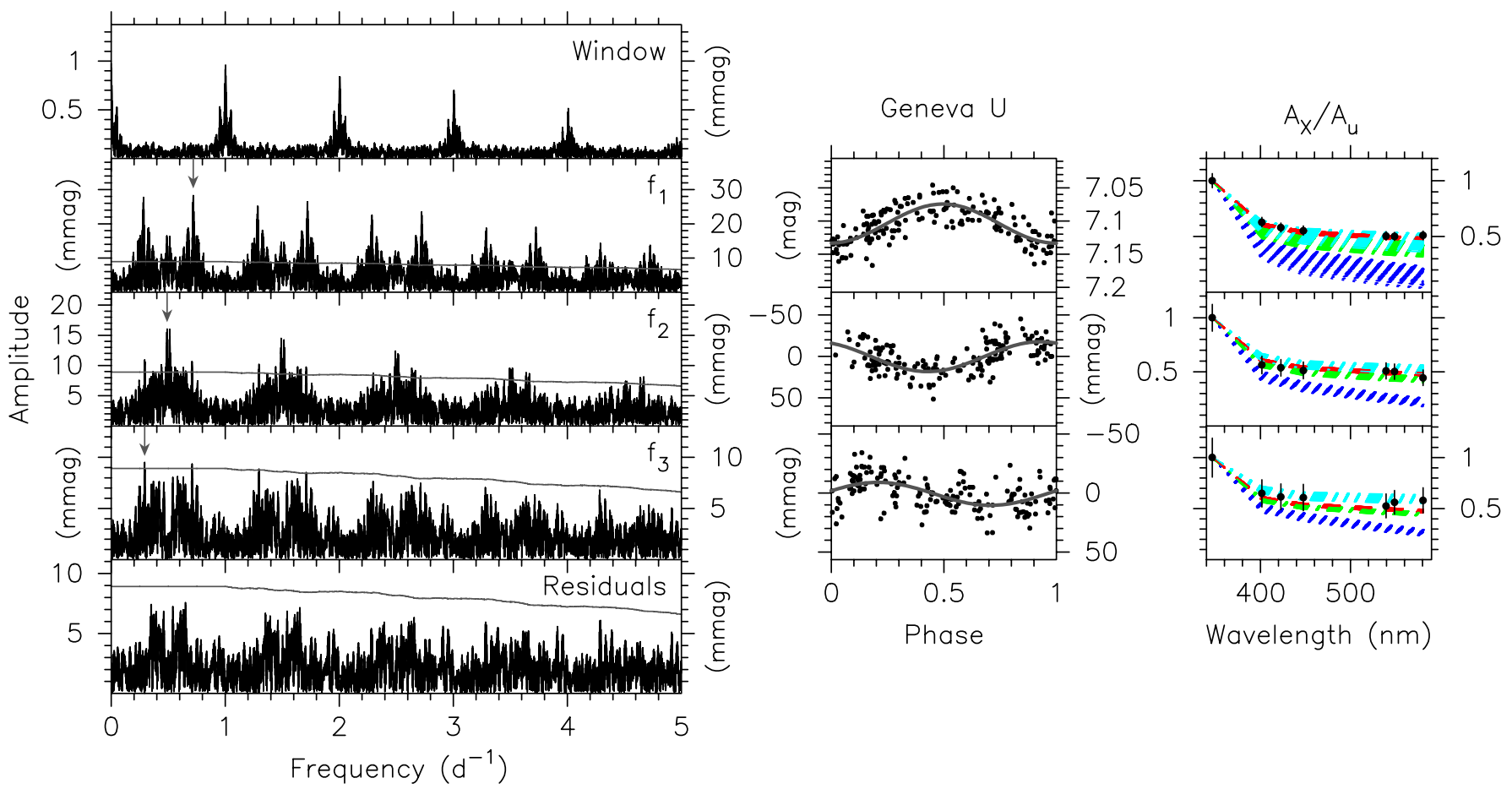

Fig. 7. Same as Fig. 2, but for $f_{1}=0.71908(6) \mathrm{d}^{-1}, f_{2}=0.49011(12) \mathrm{d}^{-1}$, and $f_{3}=0.29302(17) \mathrm{d}^{-1}$ in the GENEVA $U$ passband of HD 191295 .
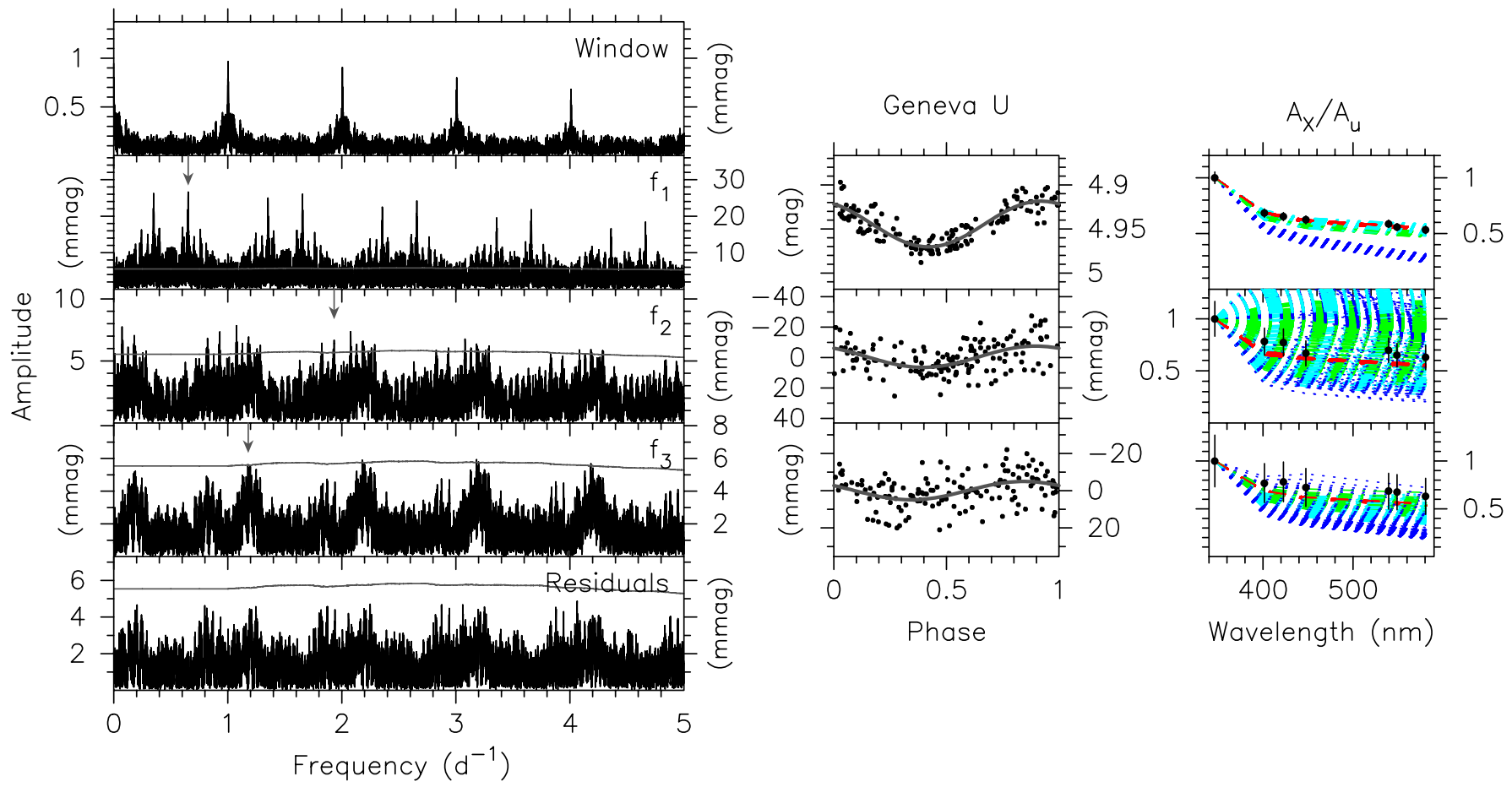

Fig. 8. Same as Fig. 2, but for $f_{1}=0.65265(2) \mathrm{d}^{-1}, f_{2}=1.93235(8) \mathrm{d}^{-1}$, and $f_{3}=1.17913(10) \mathrm{d}^{-1}$ in the GENEVA $U$ passband of HD 25558 .

low inclination angle $i$ is needed for the observed frequencies to be members of the same rotationally split mode.

The two periodic signals observed in the HIPPARCos data of HD 208057 (HIP 108022, HR 8356, OQ Peg) are also present in the MERCATOR data. Indeed, in the GENEVA $U$-filter, we detect $f_{1}=0.89050(7) \mathrm{d}^{-1}$ and $f_{2}=0.80213(10) \mathrm{d}^{-1}$. These two frequencies are not present in all the other GENEVA passbands. Moreover, we find the frequencies in a different order than in the HIPPARCos data. We additionally find evidence for $f_{3}=$
2.47585(8) $\mathrm{d}^{-1}$ or one of its aliases (Fig. 9). For both $f_{1}$ and $f_{2}$, $\ell=1$ is the most probable identification while $f_{3}$ is best fitted with an $\ell=2$ mode (Table 4 and Fig. 9, right panels). For $f_{1}$ and $f_{2}$ to be components of a rotationally split mode, we need $f_{\Omega} \leq$ $0.17 \mathrm{~d}^{-1}$, which is much lower than the lower limit $f_{\Omega} \sin i=$ $0.68(21) \mathrm{d}^{-1}$ (Table 1$)$.

The object HD 1976 (HIP 1921, HR 91, V746 Cas) is a triple system: it consists of a close visual binary with a period of 152.7 years $\left(\rho=00^{\prime \prime} 157(3), \Delta m=0.89(10)\right.$ mag at the epoch of 

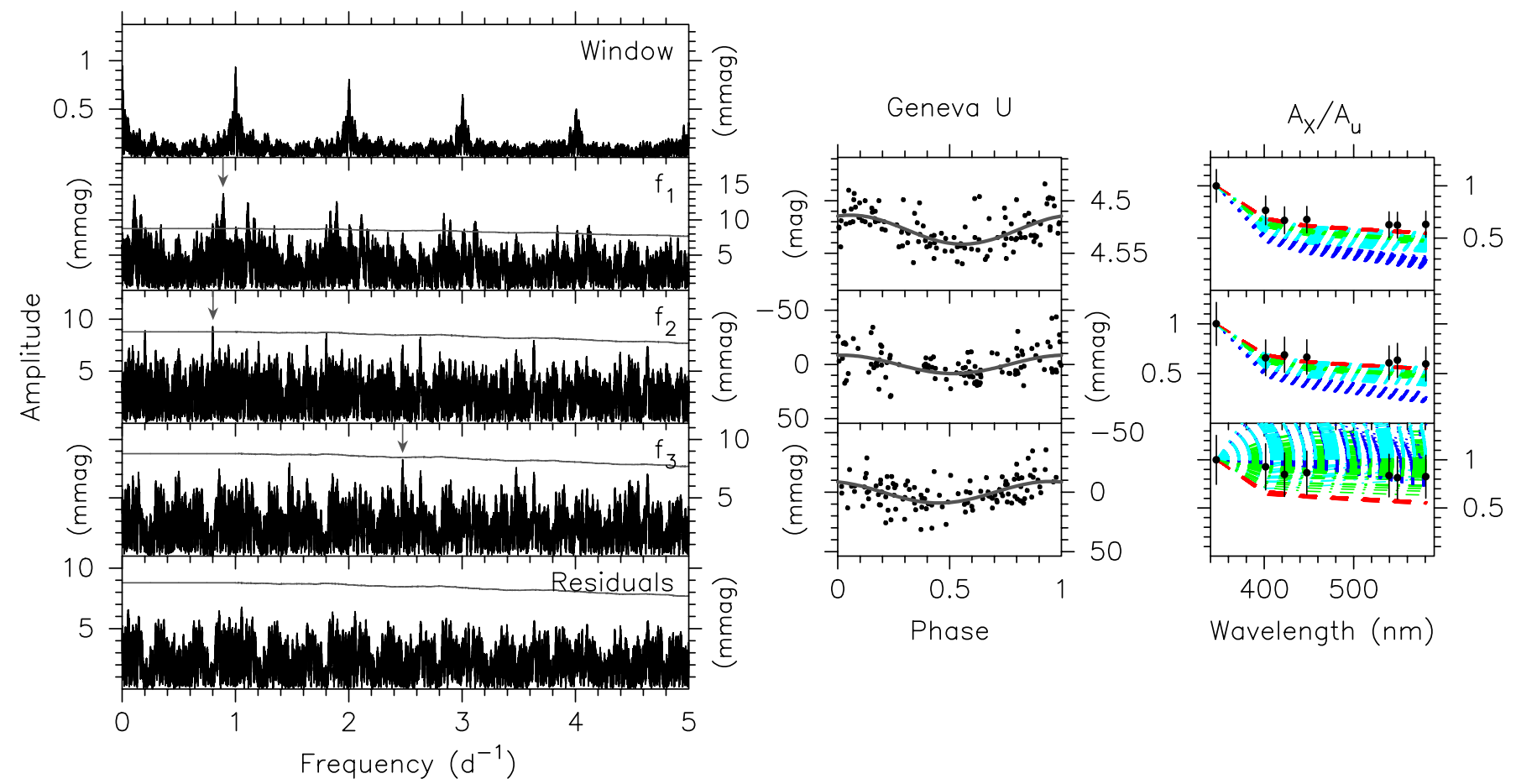

Fig. 9. Same as Fig. 2, but for $f_{1}=0.89050(7) \mathrm{d}^{-1}, f_{2}=0.80213(10) \mathrm{d}^{-1}$ and $f_{3}=2.47585(8) \mathrm{d}^{-1}$ in the GENEVA $U$ passband of HD 208057 .
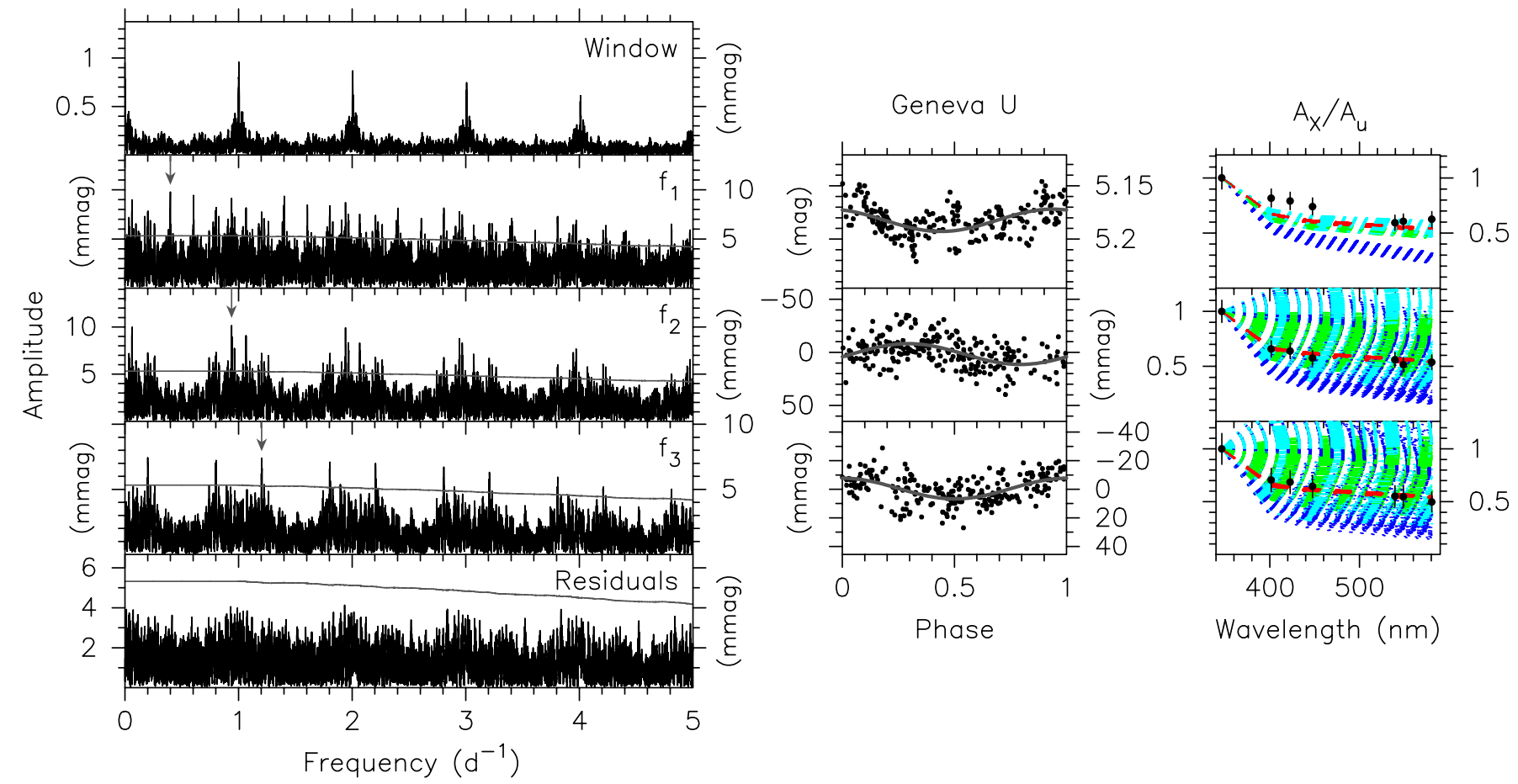

Fig. 10. Same as Fig. 2, but for $f_{1}=0.39946(4) \mathrm{d}^{-1}, f_{2}=0.93895(4) \mathrm{d}^{-1}$, and $f_{3}=1.20346(6) \mathrm{d}^{-1}$ in the GENEVA $U$ passband of HD 1976 .

the HIPPARCOS observations; ESA 1997) of which one of the components is a spectroscopic binary with a period of 27.8 (Abt \& Boonyarak 2004). It has been observed by Andrews \& Dukes (2000) with APT from late 1999 on. They found two frequencies which are compatible with those found in the HIPPARCOS data. These frequencies were not confirmed by the spectroscopic data of Mathias et al. (2001). We identified three intrinsic frequencies in the MERCATOR data, of which the first 2 coincide with those observed in the HIPPARCOS data: $f_{1}=0.39946(4) \mathrm{d}^{-1}$, $f_{2}=0.93895(4) \mathrm{d}^{-1}$, and $f_{3}=1.20346(6) \mathrm{d}^{-1}$ (Fig. 10). This is one frequency less than De Cat et al. (2004b) retained after 1.5 years of MERCATOR observations for this star. Moreover, their third frequency corresponds to $2-f_{3}$. Because HD 1976 is a multiple system, the $\log \left(T_{\text {eff }}\right)$ and $\log (g)$ values given in Table 1 , and hence the results of the mode identification should be treated with caution. The observed amplitude ratios of $f_{1}$ are not compatible with any of the considered theoretical pulsation modes with $\ell \leq 4$ and resemble those of HD 53921, which is also a 

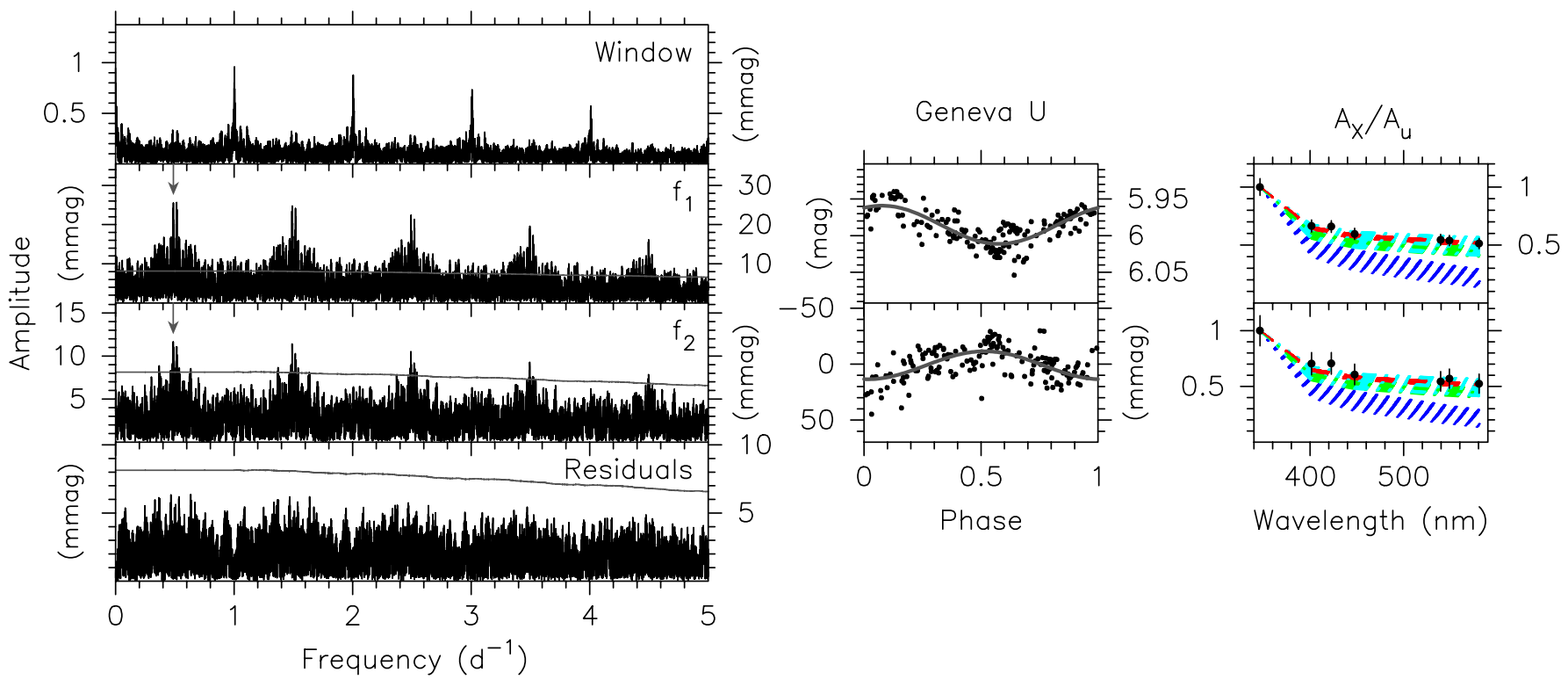

Fig. 11. Same as Fig. 2, but for $f_{1}=0.48790(4) \mathrm{d}^{-1}$ and $f_{2}=0.48666(8) \mathrm{d}^{-1}$ in the GENEVA $U$ passband of HD 28114 .
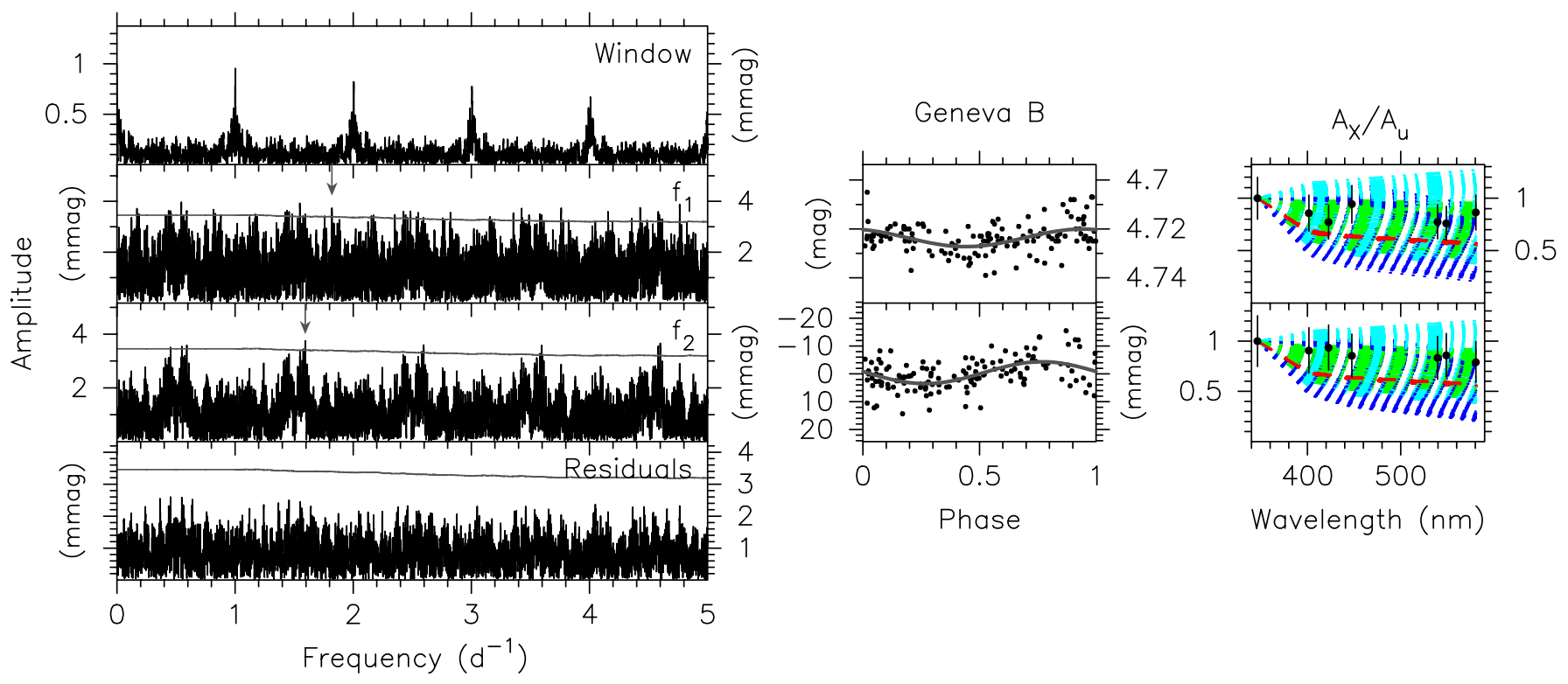

Fig. 12. Same as Fig. 2, but for $f_{1}=1.82023(15) \mathrm{d}^{-1}$ and $f_{2}=1.59418(17) \mathrm{d}^{-1}$ in the GENEVA $B$ passband of HD 3379 .

close visual binary (De Cat et al. 2005). For $f_{2}$ and $f_{3}$, the observed amplitude ratios are compatible with all considered $\ell$ values, of which $\ell=1$ is the most probable one (Table 4). Given that $f_{\Omega} \sin i=0.69(22) \mathrm{d}^{-1}$ (Table 1 ), we cannot exclude that $f_{1}$ and $f_{2}$ are components of a rotationally split mode.

For HD 28114 (HIP 20715, HR 1397, V1143 Tau), we detected two intrinsic frequencies in the MERCATOR data, i.e. $f_{1}=$ $0.48790(4) \mathrm{d}^{-1}$ and $f_{2}=0.48666(8) \mathrm{d}^{-1}$ (Fig. 11). None of these frequencies coincides with the frequency $f_{1}^{\mathrm{h}}=0.79104(23) \mathrm{d}^{-1}$ found in the HIPPARCos data. Due to the strong aliasing, we are not sure that we retained the true frequencies. Moreover, the frequency values cannot be trusted since $f_{1}$ and $f_{2}$ differ less than $1.5 / \Delta T$ (Loumos \& Deeming 1978). They are slightly different from those found in the first 18 months of data (De Cat et al. $2004 \mathrm{~b}$ ), so we may be in the process of resolving a close frequency multiplet. Indeed, both frequencies are identified as $\ell=$ 1 (or 4; Table 4 and Fig. 11, right panels) and HD 28114 is known as a slow rotator $\left(v_{\mathrm{eq}} \sin i<11 \mathrm{~km} \mathrm{~s}^{-1}\right.$; Mathias et al. 2001).
Photometric and spectroscopic variations were first reported for HD 3379 (HIP 2903, HR 155, 53 Psc = AG Psc) by Williams (1954) and Mathews (1956), respectively. The former reported a period of 0.09165 which could not be confirmed by the spectroscopic variations of the latter. Percy (1971) concluded that it is unlikely that the photometric variations of HD 3379 exceed 5 mmag. One decade later, Sareyan et al. (1979) classified HD 3379 as a $\beta$ Cep star since they observed short period variations $(0.08)$ both in photometric and spectroscopic observations, of which the amplitude might be variable. Jerzykiewicz \& Sterken (1990) ascribed the previously reported low-amplitude variations of HD 3379 to the comparison star HD 560 (34 Pcs). Wolf (1987) found a large-amplitude photometric variation with a period of about 0.096, while Le Contel et al. (1988), Balona \& Marang (1988), and Percy \& Au-Yong (2000) found HD 3379 to be constant to better than 10,2 , and 10 mmag, respectively. Le Contel et al. (2001) reported up to four low-amplitude frequencies $\left(1.81 \mathrm{~d}^{-1}, 0.11 \mathrm{~d}^{-1}, 1.22 \mathrm{~d}^{-1}, 1.57 \mathrm{~d}^{-1}\right)$ based on more than 
P. De Cat et al.: Long term photometric monitoring with the Mercator telescope, Online Material p 9
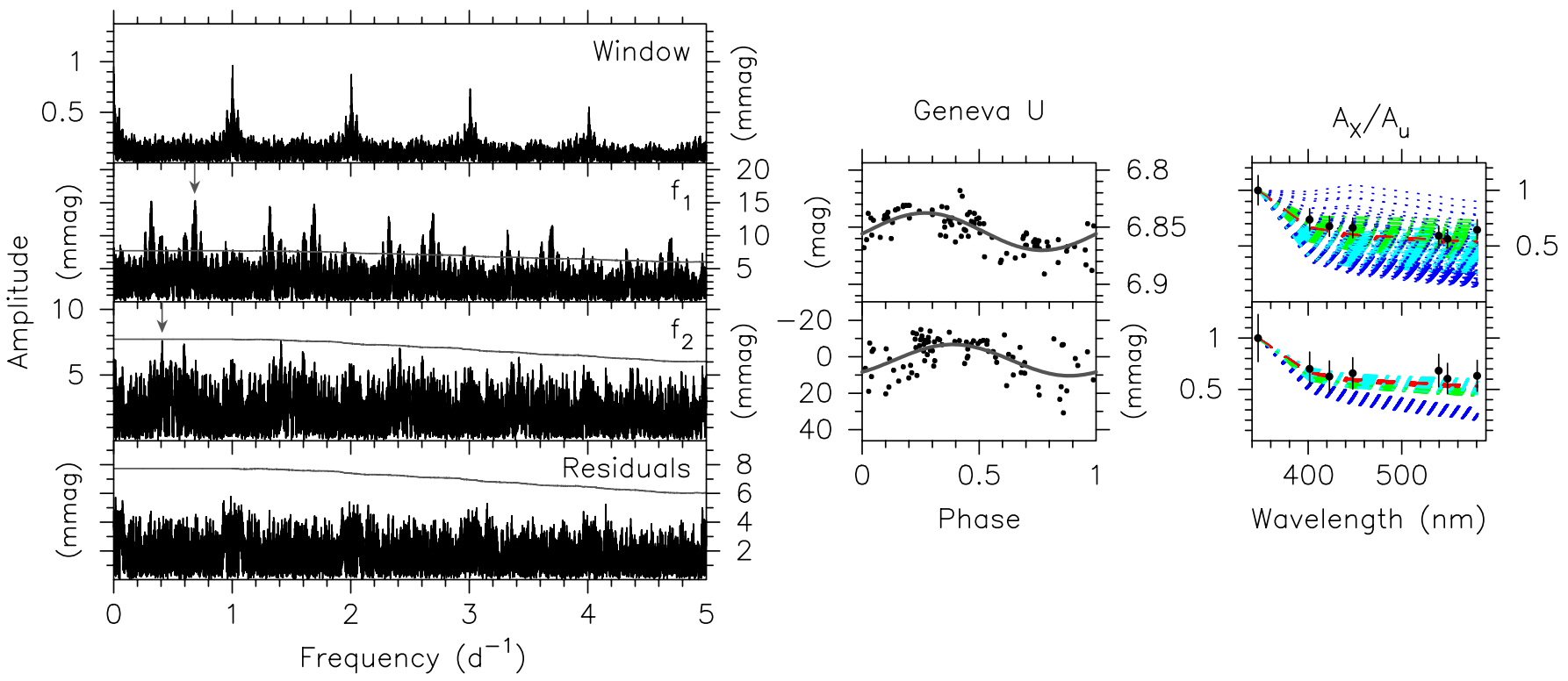

Fig. 13. Same as Fig. 2, but for $f_{1}=0.68369(7) \mathrm{d}^{-1}$ and $f_{2}=0.40893(13) \mathrm{d}^{-1}$ in the GENEVA $U$ passband of HD 28475 .
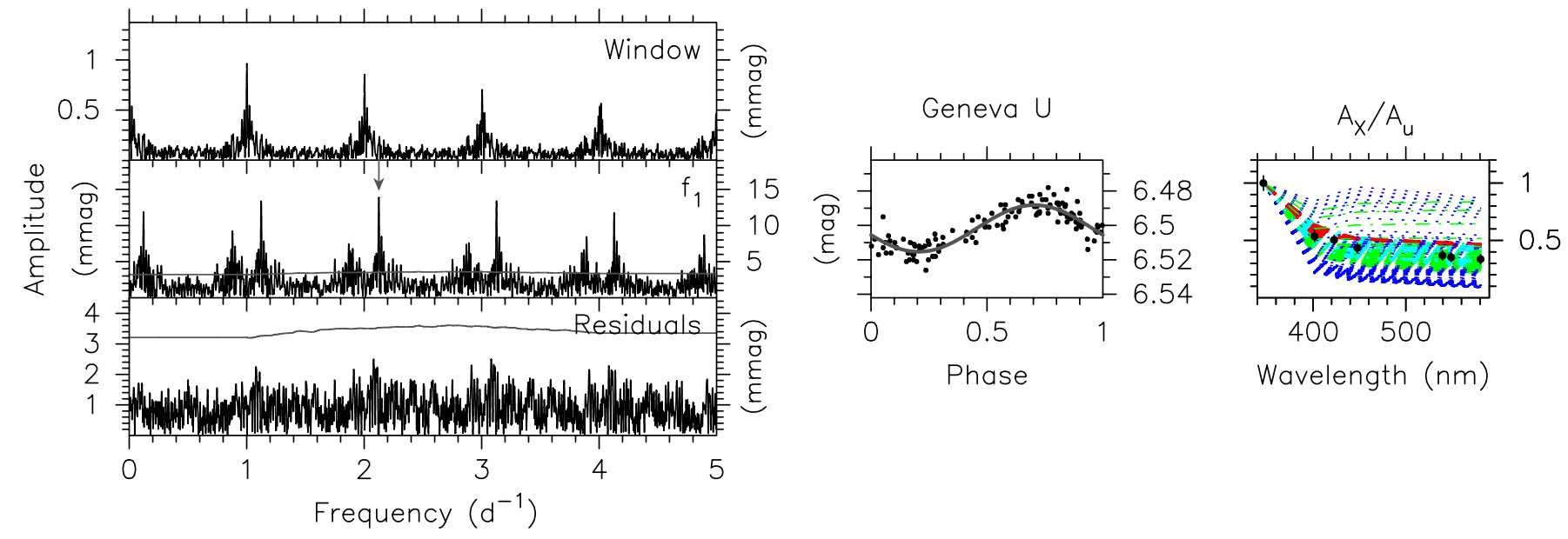

Fig. 14. Same as Fig. 2, but for $f_{1}=2.12509(32) \mathrm{d}^{-1}$ in the GENEVA $U$ passband of HD 169820.

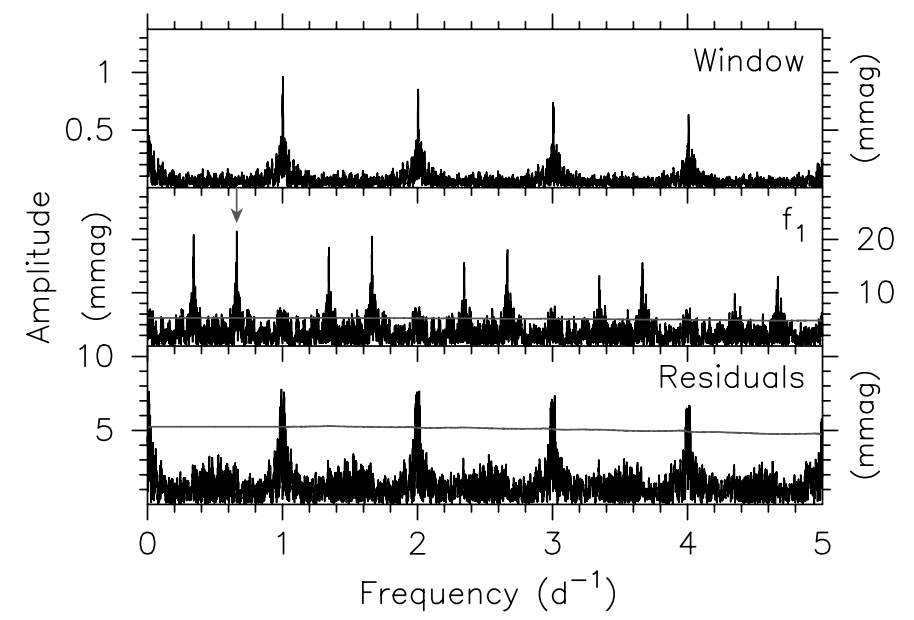

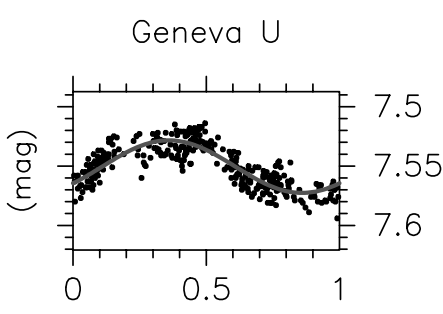

Phase

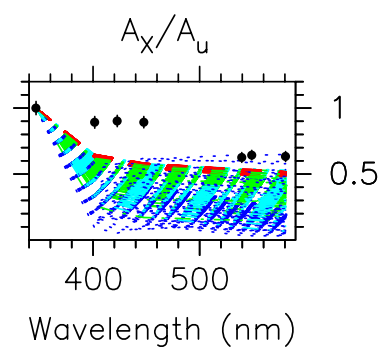

Fig. 15. Same as Fig. 2, but for $f_{1}=0.66110(3) \mathrm{d}^{-1}$ in the GENEVA $U$ passband of HD 138003.

100 spectroscopic observations with a time span of 11 . 0 . The origin of their second frequency is unclear, but they attribute the other three frequencies to SPB-pulsations. With our
MERCATOR data, there is marginal evidence for two frequencies reported by Le Contel et al. (2001), i.e. $f_{1}=1.82023(19) \mathrm{d}^{-1}$ and $f_{2}=1.59418(22) \mathrm{d}^{-1}$ (Fig. 12). We find no confirmation for any 
of these frequencies in the HIPPARCOS data. We identify both $f_{1}$ and $f_{2}$ with $\ell \geq 2$ modes (Table 4 and Fig. 12, right panels). It is plausible that $f_{1}$ and $f_{2}$ are components of a rotationally split mode with $\ell \geq 2$ because we find $f_{\Omega} \sin i=0.33(10) \mathrm{d}^{-1}$ (Table 1).

The object HD 28475 (HIP 20963, HR 1420, V1144 Tau) is known as a double-lined spectroscopic binary with an eccentricity $e=0.35$ and an orbital period of 13.8761(4). It consists of two nearly identical B5-type stars (Hube 1983). Hence, both components are potential SPBs. Because the system is completely detached, neither eclipse nor ellipsoidal variations are expected. With the MERCATOR data, we retained two frequencies: $f_{1}=$ $0.68369(7) \mathrm{d}^{-1}$ and $f_{2}=0.40893(13) \mathrm{d}^{-1}$ (Fig. 13). This confirms the suggested multi-periodic character after the analysis of the first 18 months of MERCATOR data (De Cat et al. 2004b), but our and their main frequency are aliases of each other. The first frequency is close to $f_{1}^{\mathrm{h}}=0.68537(7) \mathrm{d}^{-1}$, found as one of the highest peaks in the periodogram of the HIPPARCos data. The second frequency is new. The observed amplitude ratios of $f_{1}$ are compatible with $\ell \geq 2$, while those of $f_{2}$ can be explained with $\ell=1$ or 4 modes (Table 4 and Fig. 13, right panels). Since we are dealing with a double-lined binary, these results of the mode identification should be treated with caution. We cannot exclude that $f_{1}$ and $f_{2}$ are components of a rotationally split mode because we derived $f_{\Omega} \sin i=0.15(5) \mathrm{d}^{-1}$ from the stellar parameters of HD 28475 (Table 1).

For the Maia candidate HD 169820 (HIP 90337, HR 6906), we find $f_{1}=2.12509(32) \mathrm{d}^{-1}$ as the only frequency in the MERCATOR and HIPPARCOS data (Table 4 and Fig. 14). The $T_{\text {eff }}$ and $\log (g)$ values derived from the GENEVA photometry put HD 169820 within the theoretical instability strip of the SPBs (Table 1 and Fig. 1). The models appropriate for HD 169820 have no unstable modes in the frequency range around $f_{1}$. However, because we are dealing with a rapidly rotating star ( $v_{\mathrm{eq}} \sin i=136 \mathrm{~km} \mathrm{~s}^{-1}$; Royer et al. 2002) and since $f_{1}$ is identified with an $\ell=4$ or 2 mode (Table 4 and Fig. 14, right panel), the difference between the observed frequency and the frequency in the co-rotating frame can be large. This, in combination with the presence of $f_{1}$ in the GENEVA colours, favours the classification as a rapidly rotating SPB in analogy with HD 121190 (Aerts \& Kolenberg 2005).

For HD 138003 (HIP 75641, FK Boo), $f_{1}=0.66110(3) \mathrm{d}^{-1}$ is the only frequency found in the MERCATOR and HIPPARCOS data, as well as in the GENEVA colours (Fig. 15). Because there are unstable modes near $f_{1}$ in appropriate theoretical models for HD 138003, we have no reason to question the SPB-nature of this object. However, the observed amplitude ratios do not follow the trends predicted for pulsation modes with $\ell \leq 4$ (Fig. 15, right panel). They resemble those of HD 53921, which is a close visual binary (De Cat et al. 2005).

\subsection{2. $\beta$ Cep stars}

Hill (1967) tentatively classified the Perseus OB1-member HD 14053 (NGC 869612) as a $\beta$ Cep star. Because his data in the $U$ filter are poorly distributed in phase, the frequency $4.9458(12) \mathrm{d}^{-1}$ that was observed in the $B$ filter could not be confirmed. The stellar parameters derived from the MERCATOR data put this object well within the theoretical instability strip of the $\beta$ Ceps (Fig. 1). HD 14053 is the only object in our sample that has not been observed by the HIPPARCOS satellite. We have evidence for up to five frequencies in the MERCATOR data: $f_{1}=$ $6.08608(11) \mathrm{d}^{-1}, f_{2}=9.61805(22) \mathrm{d}^{-1}, f_{3}=7.69877(21) \mathrm{d}^{-1}$, $f_{4}=2.74081(29) \mathrm{d}^{-1}$, and $f_{5}=2.86425(80) \mathrm{d}^{-1}$, or one of their aliases (Fig. 16). We cannot exclude that Hill's frequency and $f_{1}$ are aliases. In the residual periodogram, clear frequency peaks still appear, but in each filter at different values (Fig. 16, bottom left panel). We stopped the frequency search at this point. Our frequencies need further observational confirmation, but we classify HD 14053 as a multi-periodic $\beta$ Cep star. The frequencies $f_{1}, f_{3}$, and $f_{4}$ are unambiguously identified as an $\ell=4$ mode, while the observed amplitude ratios of $f_{2}$ are compatible with both $\ell=2$ and 4 . Those of $f_{5}$ are not compatible with any of the considered theoretical modes (Table 4 and Fig. 16, right panels). Based on $f_{\Omega} \sin i=0.17(4) \mathrm{d}^{-1}$ (Table 1 ), we cannot exclude that $f_{4}$ and $f_{5}$ are components of a rotationally split mode.

Plasket \& Pearce (1930) first announced the radial velocity of HD 89688 (HIP 50684, HR 4064, 23 Sex = RS Sex) to be variable. This statement could not be confirmed nor ruled out by the observations of Sahade et al. (1956). They questioned the photometric variations reported by Walker (1952) and de Jager (1953) who classified this object as a suspected $\beta$ Cep star. Recently, Percy \& Au-Yong (2000) found HD 89688 to be constant to better than $10 \mathrm{mmag}$. The periodograms of the MERCATOR data of HD 89688 are very noisy and the aliasing is severe. We have evidence for up to four frequencies and retained $f_{1}=$ $0.79649(10) \mathrm{d}^{-1}, f_{2}=5.79148(11) \mathrm{d}^{-1}, f_{3}=10.92814(10) \mathrm{d}^{-1}$, and $f_{4}=4.09715(16) \mathrm{d}^{-1}$ (Fig. 17). There are no common peaks with the modified SCARGLE periodogram of the HIPPARCOS data, where $7.3902 \mathrm{~d}^{-1}$ and $7.7459 \mathrm{~d}^{-1}$ are the highest peaks. These frequencies both reduce the $H_{\mathrm{p}}$ variance with the same amount, so we cannot prefer one above the other. The results of the mode identification are ambiguous (Table 4 and Fig. 17, right panels). The most probable identifications for $f_{1}, f_{2}$, and $f_{4}$ are $\ell=2$, 2 , and $0 / 1$, respectively. The observed amplitudes of $f_{3}$ are inconsistent with all the considered theoretical modes with $\ell \leq 4$ (Fig. 17, right panels). Given HD 89688's position in the H-R diagram, we may be dealing with a hybrid $\beta$ Cep/SPB star (Fig. 1). However, since $f_{1}$ is close to $f_{\Omega} \sin i=0.83(20) \mathrm{d}^{-1}, f_{1}$ can easily be interpreted as $f_{\Omega}$ as well in case of a sufficiently high inclination angle $i$.

The periodic Be star ( $\lambda$ Eri star) HD 180968 (HIP 94827, HR 7318, 2 Vul = ES Vul) is known as a close visual binary with $\rho=1^{\prime \prime}$.748(12) and $\triangle m=3.55(6)$ mag at the epoch of the HIPPARCOS observations (ESA 1997). Plasket \& Pearce (1930) found the first indications of radial-velocity variations. Lynds (1959) reported a frequency of $1.6404 \mathrm{~d}^{-1}$ in a time series of yellow light observations. Stagg (1983) confirmed the short period photometric variability, but the period of Lynds (1959) did not fit his new data. A decade later, Gies (1994) reported line-profile variations with a period of $0.787 \mathrm{~d}^{-1}$. We found $f_{1}=0.63754(8) \mathrm{d}^{-1}$ and $f_{2}=2.18570(17) \mathrm{d}^{-1}$ in the MERCATOR time series (Fig. 18). The first frequency coincides with $f_{1}^{\mathrm{h}}$, which is the second highest peak in the modified SCARGLE periodogram of the HIPPARcos data. The frequency found by Lynds (1959) is a $1 \mathrm{~d}^{-1}$ alias frequency of $f_{1}$. The observed amplitude ratios of $f_{1}$ are compatible with pulsation modes with $\ell \leq 3$, and those of $f_{2}$ with $\ell \geq 2$ (Table 4 and Fig. 18, right panels). HD 180968 is situated in the middle of the instability strip of the $\beta$ Cep stars. The frequency $f_{1}$ falls outside the theoretically predicted range of unstable modes, even if the frequency shifts expected for rapid rotation are taken into account. Given that only one low frequency is found and that rotational modulation is one of the possible explanations for variations in Be stars, we prefer the interpretation of $f_{1}$ as the rotation frequency. Moreover, $f_{1}$ is fully compatible with $f_{\Omega} \sin i=$ $0.65(17) \mathrm{d}^{-1}$ (Table 1$)$.

The object HD 13831 (HIP 10615, V473 Per) is known as a $\mathrm{Be}$ star since $\mathrm{H} \alpha$ has been observed in emission 


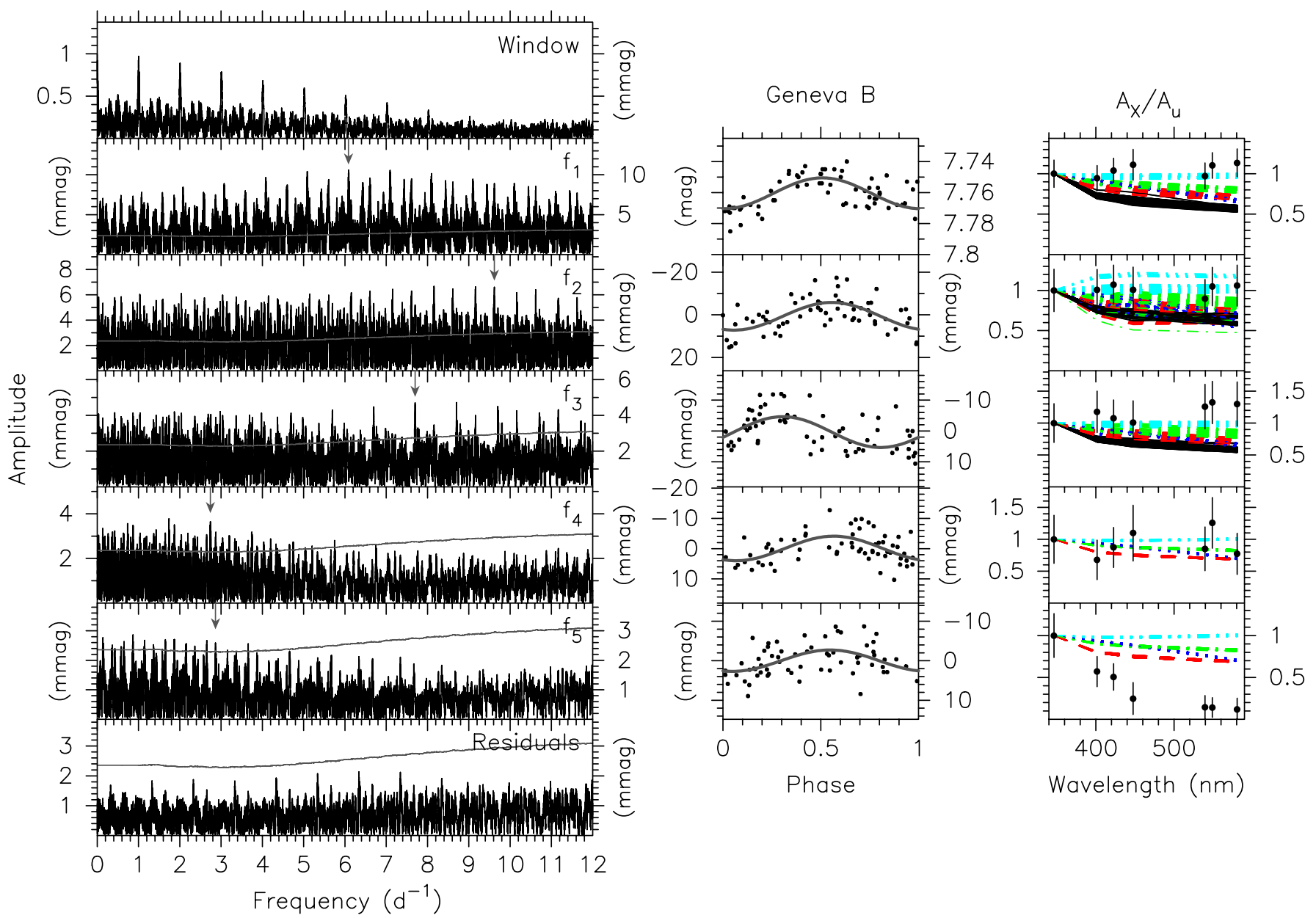

Fig. 16. Frequency analysis of the data of HD 14053 in the GENEVA $B$ passband. In the left panels, we show the window function (top), and the modified SCARGLE periodograms in the different steps of subsequent prewhitening. The detected frequencies $f_{1}=6.08608(11) \mathrm{d}^{-1}, f_{2}=$ $9.61805(22) \mathrm{d}^{-1}, f_{3}=7.69877(21) \mathrm{d}^{-1}, f_{4}=2.74081(29) \mathrm{d}^{-1}$, and $f_{5}=2.86425(80) \mathrm{d}^{-1}$ are indicated with arrows. The grey line corresponds to the $3.6 \mathrm{~S} / \mathrm{N}$-level. In the middle panels, we show phase diagrams with the detected frequencies. The dots and the grey full line denote the observations and the harmonic fit, respectively. The right panels show the results of the photometric mode identification. For each theoretical model within the observed range of $\log \left(T_{\text {eff }}\right)$ and $\log (g)$ (cf. Fig. 1 and Table 1$)$, the theoretical amplitude ratios for modes with $\ell=0,1,2,3$, and 4 are represented with a black full, a red dashed, a green dash-dotted, a blue dotted, and a cyan dash-dot-dot-dotted line, respectively. The dots indicate the observed amplitude ratios and their standard error.

(Kohoutek \& Wehmeyer 1999). Its projected rotation velocity $v_{\text {eq }} \sin i=167(28) \mathrm{km} \mathrm{s}^{-1}$ amounts to $33 \%$ of the critical break-up velocity (Clark \& Steele 2000). Hill (1967) classified HD 13831 as photometrically constant while Garrido \& Delgado (1982) observed variations with a frequency of about $5.2 \mathrm{~d}^{-1}$ with a peak-to-peak amplitude of 0.03 in the Strömgren $u, v$, and $b$ passbands, and classified it as a new $\beta$ Cep star. In the HIPPARCOS catalogue, it is listed as an unsolved variable. In the MERCATOR data, we have evidence for two frequencies: $f_{1}=0.97525(8) \mathrm{d}^{-1}$ and $f_{2}=5.41824(11) \mathrm{d}^{-1}$, or one of their aliases (Fig. 19). The $\beta$ Cep-like variations observed by Garrido $\&$ Delgado (1982) are confirmed by $f_{2}$. Because there is no intersection between the $\left(\log \left(T_{\text {eff }}\right), \log (g)\right)$ error box and our grid of models, we are unable to identify the modes (Fig. 19, right panels). As for HD 180968, we interprete the low frequency $f_{1}$ as the rotation frequency of HD 13831.

The first O-star in our sample, HD 214680 (HIP 111841, HR 8622, 10 Lac), is the most massive member of the Lacertae OB Ib association (Humphreys 1978). Murdoch et al. (1994) report the presence of $\mathrm{Br} \alpha$ and $\mathrm{Br} \gamma$ emission features in the infrared spectrum of this O9 V MK standard. An overview of its properties is given by Brandt et al. (1998). HD 214680 has a vi- sual component of $10 \mathrm{mag}$ and a separation $\rho=62 . " 3$ at the epoch of the observations of Mason et al. (1998). Line-profile and/or radial-velocity variations were already observed on several occasions (e.g. Grygar 1963; Underhill \& van Helden 1966; Chochol \& Grygar 1976) before Smith (1978) classified HD 214680 as a 53 Persei star (most members of this class are now reclassified as SPBs). He found a frequency of $4.89 \mathrm{~d}^{-1}$ in line-profile variations which he ascribed to an $\ell=2$ sectoral mode. Smith also found evidence that it concerns a complex pulsator capable of changing its amplitude and period on a time scale of months. Migrating discrete absorption components are observed in the UV resonance doublets, with a reoccurrence timescale of about 7 days (Kaper et al. 1999 and references therein). Photometric variability with an amplitude between 20 and 30 mmag and a candidate frequency of $3.75 \mathrm{~d}^{-1}$ have been reported by Delgado \& Garrido (1981), but this has never been confirmed. Our MERCATOR data gives evidence for periodic photometric variations with $f_{1}=3.2584(8) \mathrm{d}^{-1}$ (Fig. 20). It cannot be excluded that Delgado \& Garrido's frequency and $f_{1}$ are aliases. No identification could be performed for this star because the full $\left(T_{\text {eff }}, \log (g)\right)$ error box of HD 214680 falls outside the grid of models. We recall that HD 214680 is the brighest object of our sample and that 
P. De Cat et al.: Long term photometric monitoring with the Mercator telescope, Online Material $p 12$
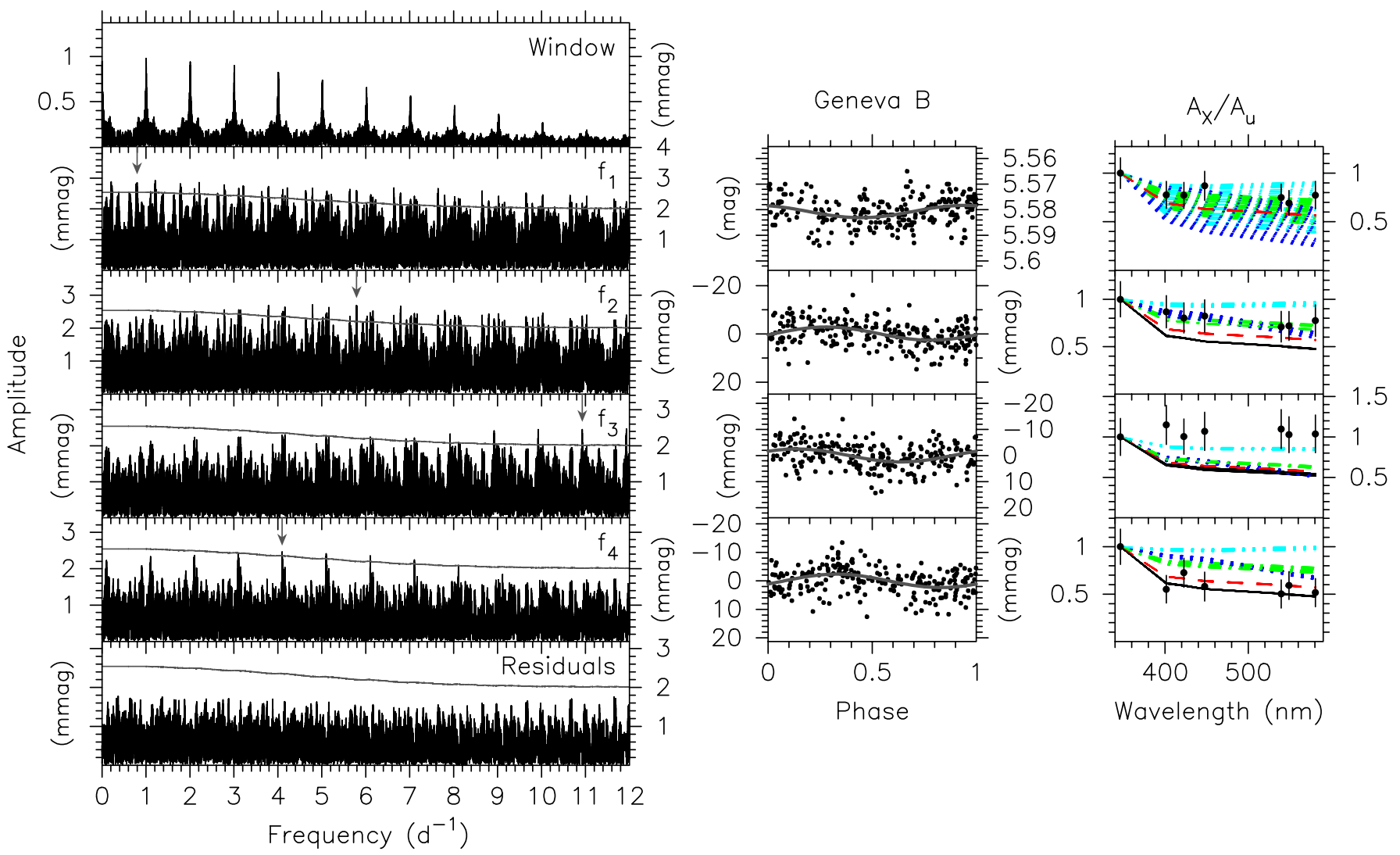

Fig. 17. Same as Fig. 16, but for $f_{1}=0.79649(10) \mathrm{d}^{-1}, f_{2}=5.79148(11) \mathrm{d}^{-1}, f_{3}=10.92814(10) \mathrm{d}^{-1}$, and $f_{4}=4.09715(16) \mathrm{d}^{-1}$ in the GENEVA $B$ passband of HD 89688.
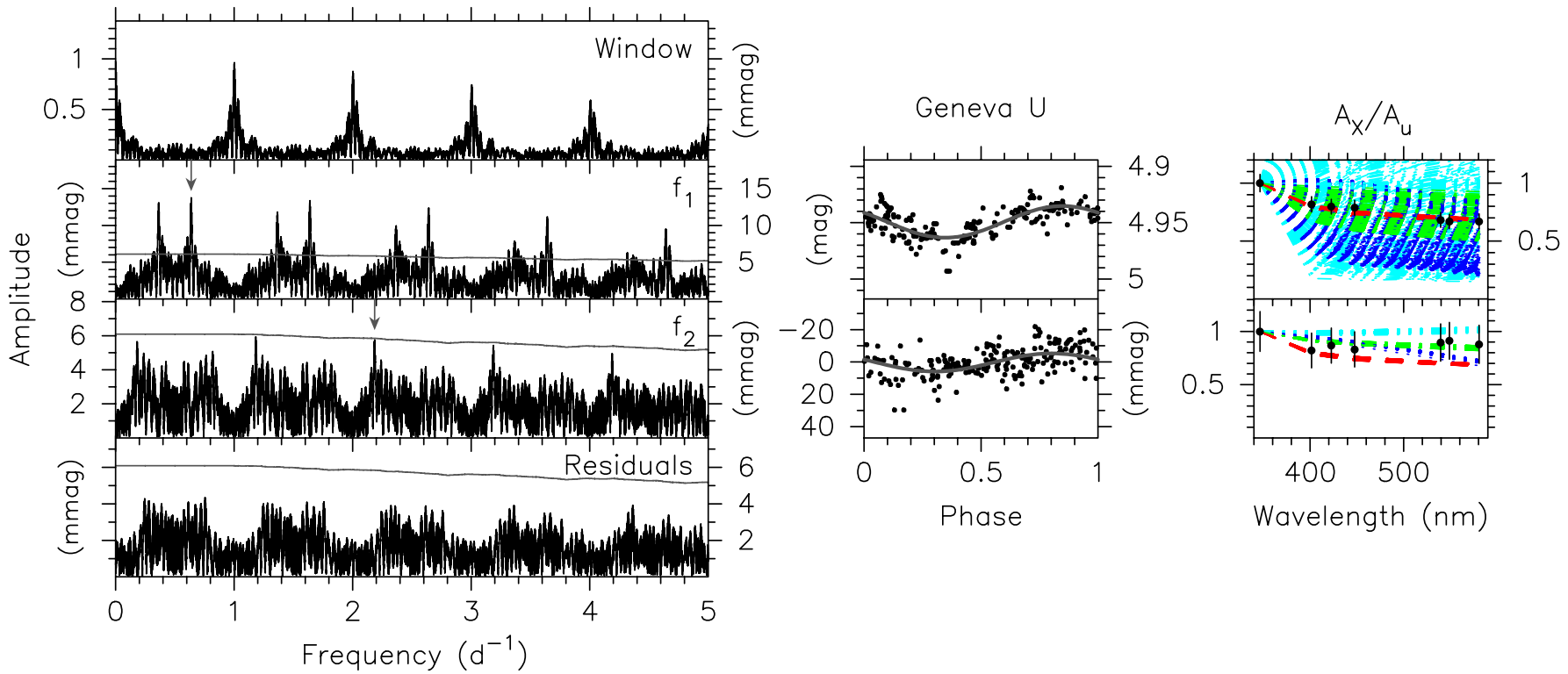

Fig. 18. Same as Fig. 16, but for $f_{1}=0.63754(8) \mathrm{d}^{-1}$ and $f_{2}=2.18570(17) \mathrm{d}^{-1}$ in the GENEVA $U$ passband of HD 180968 .

a significant amount of data was rejected because of the poor quality. Although our results should be treated with caution, we now consider HD 214680 as a $\beta$ Cep star.

\subsubsection{Hybrid $\beta$ Cep/SPB stars}

The second O-star in our sample is HD 13745 (HIP 10541, V354 Per). Hill (1967) found light variations for this object with a frequency of 2.2203(4) $\mathrm{d}^{-1}$ and classified it as a new $\beta$ Cep. Based on the HIPPARCOS data, it was first classified as a constant star, but later reclassified as a periodic variable with frequency 11.44972(6) $\mathrm{d}^{-1}$ by Koen \& Eyer (2002). We have evidence for the presence of four frequencies in the MERCATOR data: $f_{1}=$ $1.13358(6) \mathrm{d}^{-1}, f_{2}=2.64441(6) \mathrm{d}^{-1}, f_{3}=0.68063(7) \mathrm{d}^{-1}$, and $f_{4}=8.18548(11) \mathrm{d}^{-1}$, or one of their aliases (Fig. 21). None of these frequencies coincide with frequencies previously reported in the literature. Because $f_{1}, f_{2}$, and $f_{3}$ are SPB frequencies 

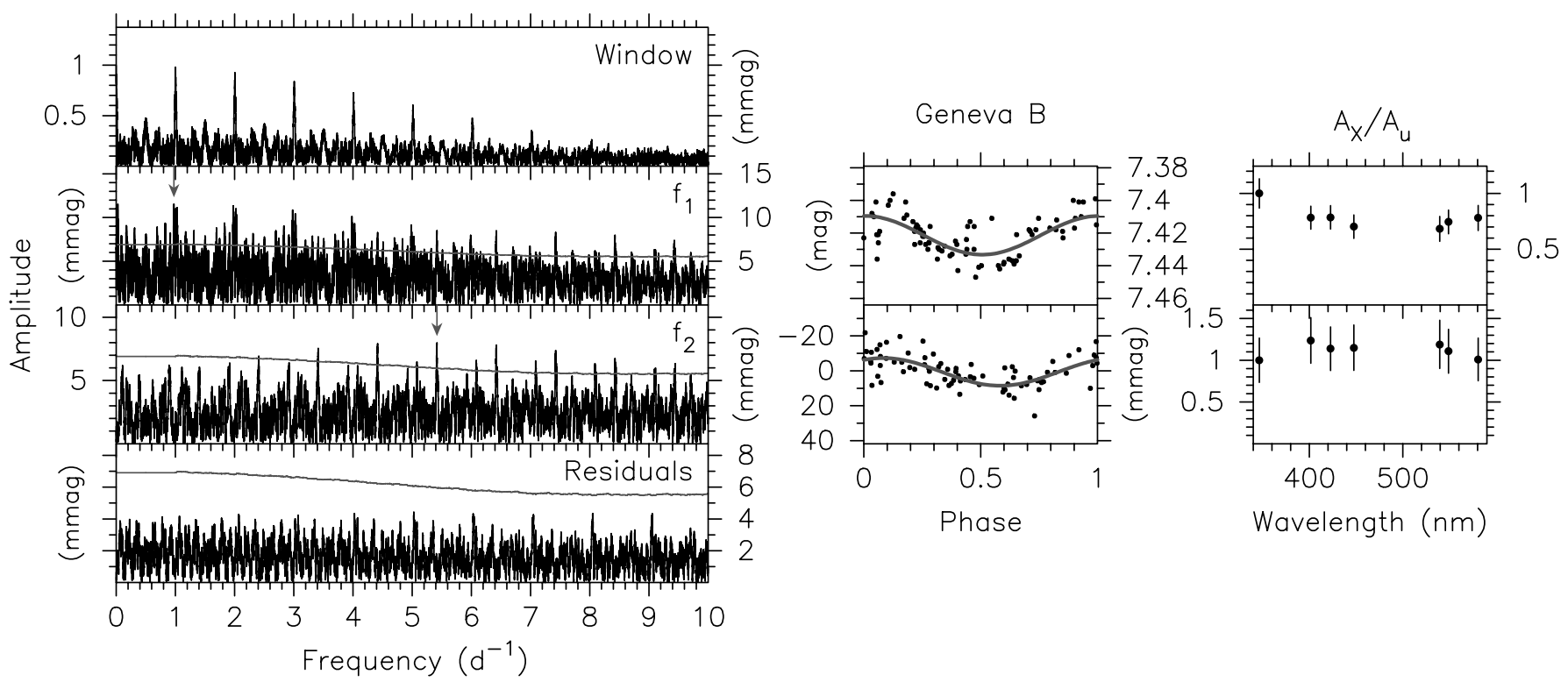

Fig. 19. Same as Fig. 16, but for $f_{1}=0.97525(8) \mathrm{d}^{-1}$ and $f_{2}=5.41824(11) \mathrm{d}^{-1}$ in the GENEVA $B$ passband of HD 13831 .
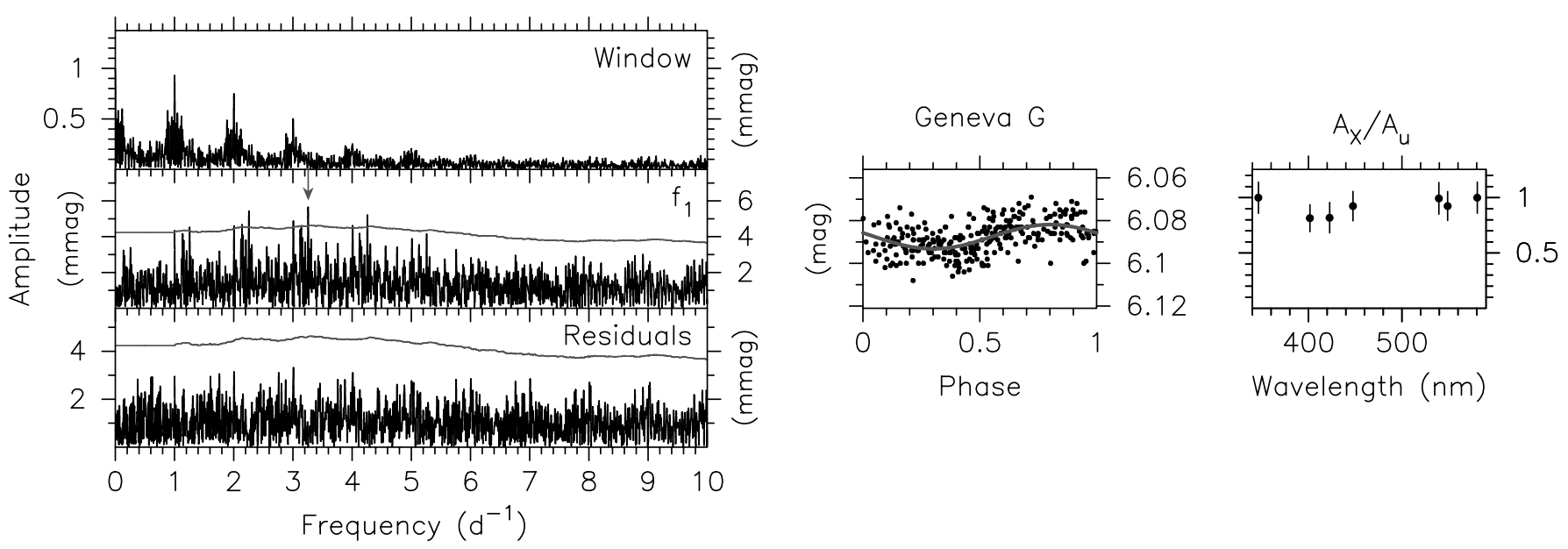

Fig. 20. Same as Fig. 16, but for $f_{1}=3.2584(8) \mathrm{d}^{-1}$ in the GENEVA $G$ passband of HD 214680 .

and $f_{4}$ is a $\beta$ Cep frequency, we classify HD 13745 as a hybrid $\beta \mathrm{Cep} / \mathrm{SPB}$ star. This is consistent with its position in the H-R diagram which is rather close to the hot common theoretical $\beta$ Cep/SPB instability strip predicted by e.g. Pamyatnykh (1999) (Table 1 and Fig. 1). Unfortunately, none of the models of our grid falls within the observed error box of HD 13745, so we are unable to perform mode identification for this star (Fig. 21, right panels). Given that the observed amplitude ratios resemble those of e.g. HD 3379 ( $g$-modes; Fig. 12) or HD 14053 ( $p$ modes; Fig. 16), we are convinced that they are compatible with $p$ - and/or $g$-modes.

The star HD 19374 (HIP 14514, HR 938, 53 Ari = UW Ari) is a runaway star from the I Ori association (Blaauw 1956) that is situated along the border of the theoretical instability strip of the $\beta$ Ceps (Fig. 1). Its behaviour is controversial. On the one hand, the spectroscopic observations of Münch \& Flather (1957) and Van Hoof \& Blaauw (1964) and the photometric observations of Bondal (1967) all showed variations which are compatible with a frequency of about $6.5 \mathrm{~d}^{-1}$, leading to the classification as a $\beta$ Cep. On the other hand, the spectroscopic and photometric observations of Lane (1976) and Jerzykiewicz (1974), respectively, revealed no significant variations. Sterken (1988) observed a large scatter in radial velocity on a time scale of a few hours which may be caused by line-profile variations, but he rules out the classification as a $\beta$ Cep and prefers an interpretation as a "totally disrupted system". Gonzalez Bedolla (1994) reobserved this star photometrically during three nights. He observed periodic variations only during the night in 1980 and a flash of about one hour during the last night in 1982. Therefore, a peculiar amplitude modulation or transient variability may better account for the observations of this star. Adelman (2001) included HD 19374 in his list of the "least variable stars" observed with the HIPPARCOS satellite. The MERCATOR data gives evidence for three frequencies: $f_{1}=1.32663(4) \mathrm{d}^{-1}, f_{2}=6.50027(9) \mathrm{d}^{-1}$, and $f_{3}=4.36573(12) \mathrm{d}^{-1}$, or one of their aliases (Fig. 22). The frequency previously mentioned in the literature is confirmed by $f_{2}$. A frequency as low as $f_{1}$ is not predicted for models compatible with the position of HD 19374 in the H-R diagram. Given that $v_{\text {eq }} \sin i=5 \mathrm{~km} \mathrm{~s}^{-1}$ (Abt et al. 2002), no large frequency shifts are expected for the non-zonal components of non-radial modes. Moreover, $f_{1}$ is unlikely to be the rotation frequency $f_{\Omega}$ because $f_{\Omega} \sin i$ is very low (Table 1 ). The available spectroscopic studies do not allow us to relate $f_{1}$ to binary motion. We therefore tentatively classify HD 19374 as a hybrid $\beta$ Cep/SPB star. Because an 
P. De Cat et al.: Long term photometric monitoring with the Mercator telescope, Online Material $p 14$
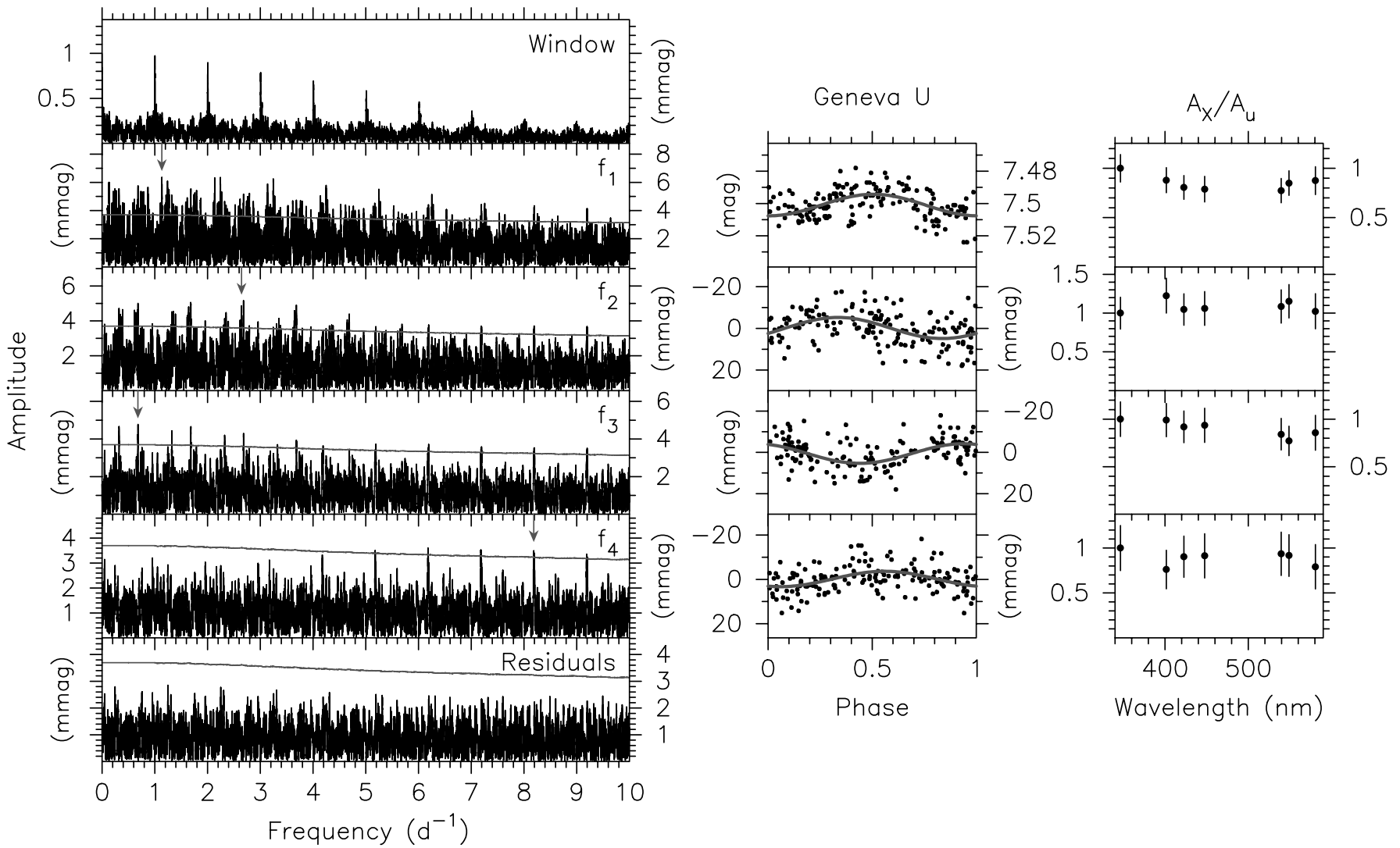

Fig. 21. Frequency analysis of the data of HD 13745 in the GENEVA $U$ passband. In the left panels, we show the window function (top), and the modified SCARGLE periodograms in the different steps of subsequent prewhitening. The detected frequencies $f_{1}=1.13358(6) \mathrm{d}^{-1}, f_{2}=$ $2.64441(6) \mathrm{d}^{-1}, f_{3}=0.68063(7) \mathrm{d}^{-1}$, and $f_{4}=8.18548(11) \mathrm{d}^{-1}$ are indicated with arrows. The grey line corresponds to the $3.6 \mathrm{~S} / \mathrm{N}-l e v e l$. In the middle panels, we show phase diagrams with the detected frequencies. The dots and the grey full line denote the observations and the harmonic fit, respectively. In the right panels, the dots indicate the observed amplitude ratios and their standard error. No identification could be performed for this star because the full $\left(T_{\text {eff }}, \log (g)\right)$ error box of HD 13745 falls outside the grid of models.

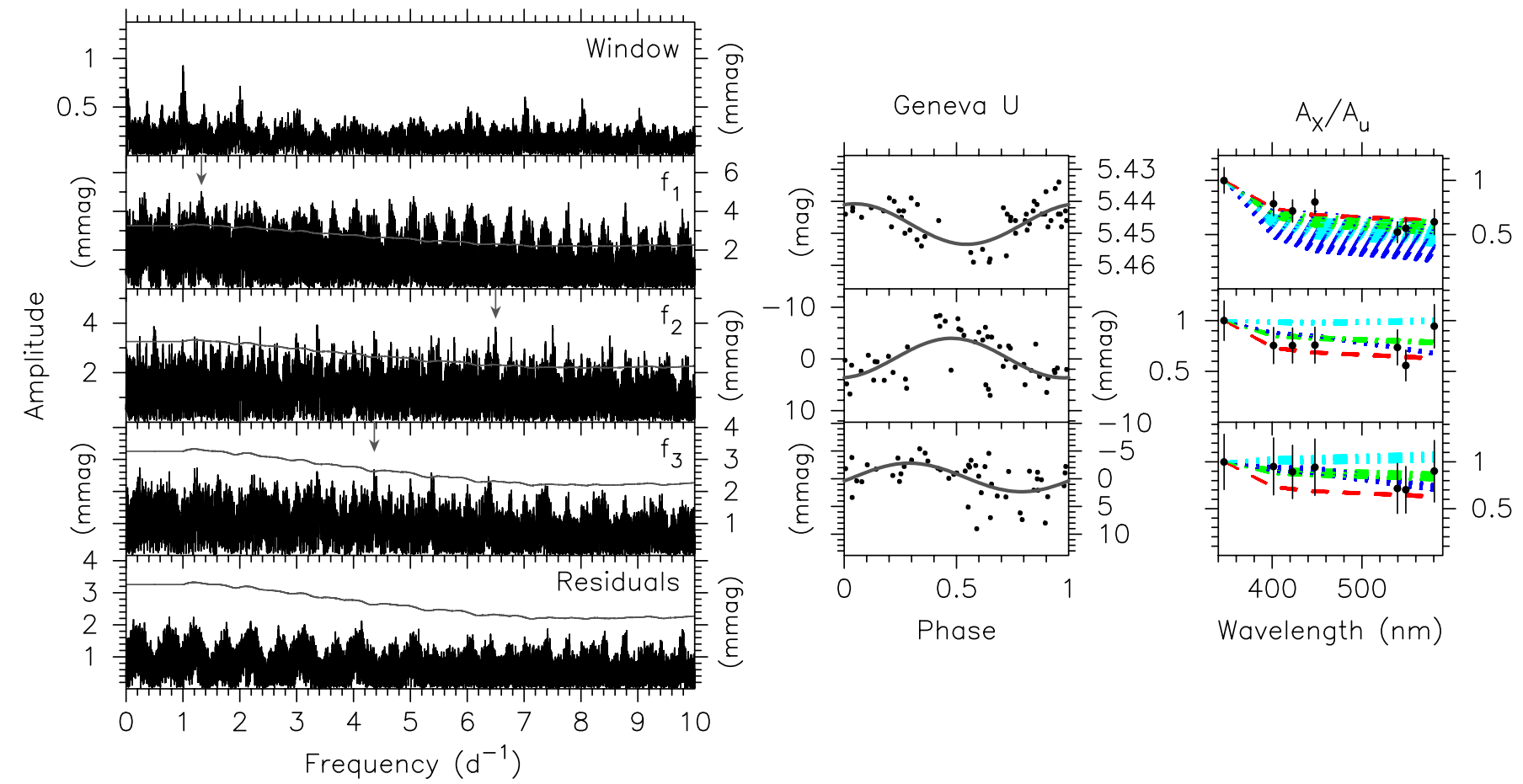

Fig. 22. Same as Fig. 21, but for $f_{1}=1.32663(4) \mathrm{d}^{-1}, f_{2}=6.50027(9) \mathrm{d}^{-1}$, and $f_{3}=4.36573(12) \mathrm{d}^{-1}$ in the GENEVA $U$ passband of HD 19374. The right panels show the results of the photometric mode identification. For each theoretical model within the observed $\operatorname{range}$ of $\log \left(T_{\text {eff }}\right)$ and $\log (g)$ (cf. Fig. 1 and Table 1), the theoretical amplitude ratios for modes with $\ell=1,2,3$, and 4 are represented with a red dashed, a green dash-dotted, a blue dotted, and a cyan dash-dot-dot-dotted line, respectively. The dots indicate the observed amplitude ratios and their standard error. 
extrapolation of the calibration tables was needed for the determination of $T_{\text {eff }}$ and $\log (g)$ of HD 19374, its position in the H-R diagram (Fig.1) and hence also the results of the mode identification should be treated with caution. We only find compatibility between the observed amplitude ratios of $f_{3}$ and modes with $\ell \leq$ 3 (Table 4 and Fig. 22, right panels).

\subsection{Non-pulsating variables}

For the six stars in this section, we argue that they were misclassified as pulsating variables. We prefer a classification as an ellipsoidal variable or a spotted star instead. It is not always clear which of the two should be preferred, and our classification should be checked with high-resolution spectroscopic data. We will also use the method of amplitude ratios for these nonpulsating variables to check how good/bad the behaviour of the amplitude ratios can be discriminated from pulsation modes.

\subsubsection{Binaries}

For four objects, we have strong indications that we are dealing with binaries instead of pulsators. The clearest case is HD 208727 (HIP 108348, HR 8377, V2173 Cyg) which is a rapidly rotating star for which the HIPPARCOS team found a frequency of $3.16778(3) \mathrm{d}^{-1}$. This object was therefore classified as a suspected SPB (Waelkens et al. 1998) and later on as a suspected Maia star. The values of $T_{\text {eff }}$ and $\log (g)$ derived from the GENEVA photometry put HD 208727 within the theoretical instability strip of the SPBs (Fig. 1). Recently, Kallinger et al. (2002) obtained 70 additional Stromgren $b$ and $y$ observations and confirmed the HIPPARCos frequency. They found no evidence for additional variability and tentatively attribute the observed variations to rotational modulation since no unstable modes are found in this frequency range for a model with similar parameters to those of HD 208727. It is clear that HD 208727 is an ellipsoidal variable instead. Indeed, we found $f_{1}=3.16780(1) \mathrm{d}^{-1}$ and $f_{2}=1.58344(10) \mathrm{d}^{-1} \simeq \frac{1}{2} f_{1}$ as the first and second frequency in the MERCATOR data. Optimisation yields $f_{\text {orb }}=1.58390(10) \mathrm{d}^{-1}$ (Fig. 23). Variations with $2 f_{\text {orb }}$ are very dominant in the GENEVA passbands, while $f_{\text {orb }}$ is the only frequency present in the GENEVA colours. In periodograms of the HIPPARCos $H_{\mathrm{p}}$ data, the only significant peak corresponds to $3.16778(3) \mathrm{d}^{-1} \simeq 2 f_{\text {orb}}$. After prewhitening, there is no evidence of intrinsic variability. If the system were synchronised, the orbital inclination $i$ would be close to $90^{\circ}$ because $f_{\Omega} \sin i=$ $1.62(45) \mathrm{d}^{-1}$ (Table 1). However, we have no observational evidence of eclipses. The observed amplitude ratios of HD 208727 corresponding to the dominant frequency $f_{1}$ are all close to 1 (Fig. 23, right panel). Only the selected $\ell=2$ modes of a few models are compatible with them. This is no surprise because a sectoral $\ell=2$ mode pulsating with $2 f_{\text {orb }}$ mimics an ellipsoid rotating with $f_{\text {orb }}$.

In the H-R diagram, the object HD 24094 (HIP 18151, CY Cam) is located close to the theoretical instability strip of the $\beta$ Ceps (leftmost filled triangle in Fig. 1), while the main frequency observed in the HIPPARCOS data is characteristic of SPBs. It is classified as a contact binary with hot components for which a good light-curve is lacking, but with frequent observations of minima which allow the determination of a reliable ephemeris (Pribulla et al. 2003). With our MERCATOR data, we find $f_{1}=1.90105(2) \mathrm{d}^{-1}$ as the only periodic signal in the GENEVA light curves. It reduces the variance in the data of the passbands with $90 \%$ or more, but it is not present in the GENEVA colours. We have no evidence for the presence of a periodic signal with $\frac{1}{2} f_{1}$ in the MERCATOR data. Optimisation of this frequency yields $f_{\text {orb }}=0.95053(14) \mathrm{d}^{-1}$ (Fig. 24), which only has a small but significant amplitude in the $U, B_{1}, B$ and $G$ filter. Hence, the classification as a contact binary cannot be confirmed or ruled out. If the variations with $f_{1}$ are interpreted in terms of pulsations, then we find compatibility between the observed and theoretical amplitude ratios of $\ell=2$ or 3 modes (Fig. 24, right panel).

For HD 112396 (HIP 63096), one frequency is clearly present in the data of the GENEVA passbands: $f_{1}=$ $1.41491(4) \mathrm{d}^{-1}$. This frequency coincides with the main frequency in the HIPPARCos data. The most dominant peaks in the periodogram of the residual MERCATOR data are close to integer values and remain below the $3.6 \mathrm{~S} / \mathrm{N}$-level. There are no longer any dominant peaks in the residual HIPPARCOS data. Hence, there is no evidence for multi-periodicity. Given that variations with $f_{1}$ are not significantly present in the GENEVA colours and that the peak-to-peak amplitude of three $v_{\text {rad }}$ observations of Fehrenbach et al. (1987) amounts to $105 \mathrm{~km} \mathrm{~s}^{-1}$, we tentatively classify HD 112396 as an ellipsoidal variable with an orbital frequency $f_{\text {orb }}=0.70745(21) \mathrm{d}^{-1}$ (Fig. 25). This suggestion is strengthened by the observation that the amplitude ratios cannot be succesfully interpreted in terms of pulsation modes with $\ell \leq 4$ (Fig. 25, right panel) and that HD 112396 is situated outside the theoretical instability strips of both the SPBs and $\beta$ Ceps (Fig. 1, rightmost filled triangle).

The radial-velocity variations of HD 149881 (HIP 81362, V600 Her) were first detected by Plasket \& Pearce (1930). Lynds (1959) observed short-periodic light variations $\left(P \sim 0^{d} .2\right)$ and first classified it as a $\beta$ Cep star. Hill et al. (1976) studied HD 149881 both photometrically and spectroscopically and concluded that this object is an ellipsoidal variable with an orbital period of $P_{\mathrm{orb}}=5$.20065(2). They attributed the additional period of $P \sim 0$. 18 to $\beta$ Cep pulsations of the primary component, which recently evolved off the main sequence (Koch 1990). With the MERCATOR data, we can confirm the classification of HD 149881 as an ellipsoidal variable because we have evidence for $f_{1}=0.38445(9) \mathrm{d}^{-1}$ in some of the GENEVA passbands, which corresponds to $P_{\text {orb }} / 2$ (Fig. 26). Like HD 112396, an interpretation of $f_{1}$ in terms of pulsation fails (Fig. 26, right panel). After optimisation, we find $f_{\text {orb }}=0.19223(53) \mathrm{d}^{-1}$. After prewhitening, there are only significant peaks at integer frequencies in the GENEVA passbands (Fig. 26, bottom left panel). Hence, we do not have evidence for pulsations in the primary component. In the HIPPARCOS data, no significant frequency peaks are found.

\subsubsection{Spotted stars}

Although HD 154689 (HIP 83467) has been flagged as an "unsolved" variable in the HIPPARCos catalogue, Koen \& Eyer (2002) classified it as a new suspected periodic variable because they found evidence for the frequency $3.91545 \mathrm{~d}^{-1}$. Because of its position in the H-R diagram, we considered it as a suspected Maia star (right star in Fig. 1). With the new MERCATOR data, we find the same main frequency $f_{1}=3.91587(81) \mathrm{d}^{-1}$ (Fig. 27). However, this signal is not significantly present in the GENEVA colours (except for $[U-B]$ ). The amplitude of $f_{1}$ increases towards the red filters and cannot be modelled with pulsation modes with $\ell \leq 4$ (Fig. 27, right panel). Moreover, one of the best candidates for the second frequency is $f_{1} / 2$. We suspect that we are dealing with rotational modulation instead of stellar pulsation, and that $f_{1} / 2$ corresponds to the rotation 
P. De Cat et al.: Long term photometric monitoring with the Mercator telescope, Online Material p 16
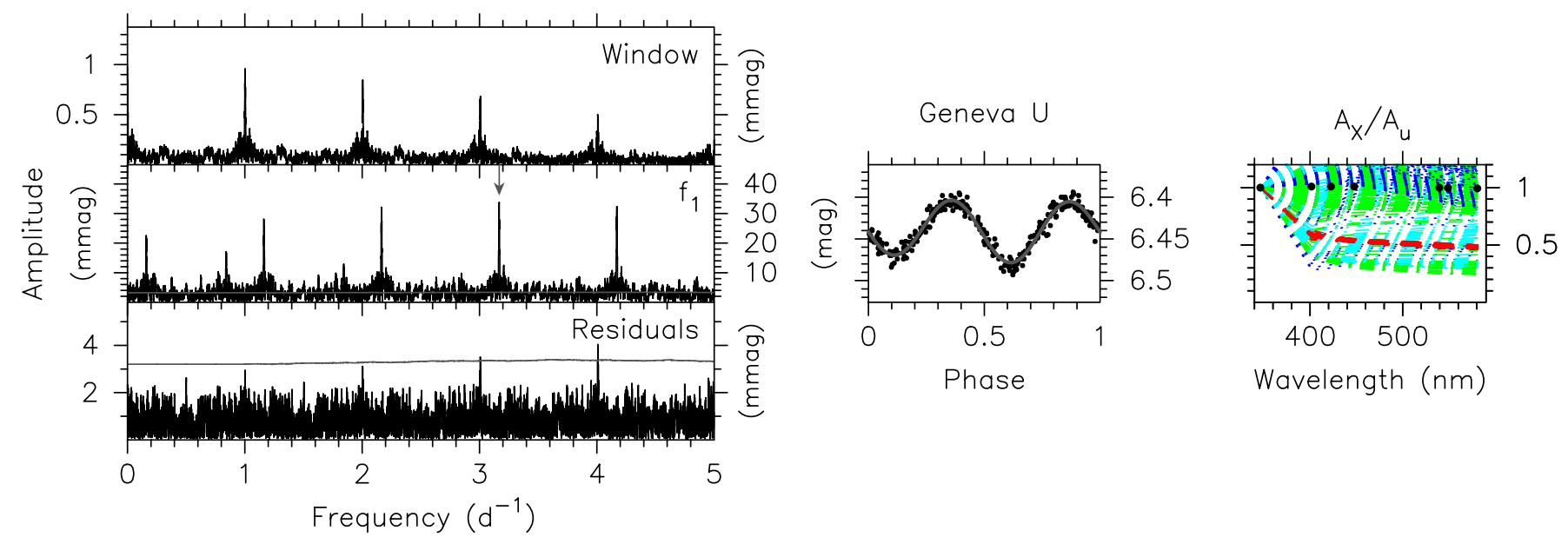

Fig. 23. Frequency analysis of the data of HD 208727 in the GENEVA $U$ passband. In the left panels, we show the window function (top), and the modified SCARGLE periodograms before and after prewhitening with $f_{\text {orb }}=1.58390(10) \mathrm{d}^{-1}$ and $2 f_{\text {orb }}$. The dominant frequency $f_{1}=2 f_{\text {orb }}$ is indicated with an arrow. The grey line corresponds to the $3.6 \mathrm{~S} / \mathrm{N}$-level. In the middle panel, we show a phase diagram with $f_{\text {orb }}$. The dots and the grey full line denote the observations and the harmonic fit, respectively. The right panel shows the results of the interpretation of the dominant frequency in terms of pulsation. For each theoretical model within the observed range of $\log \left(T_{\text {eff }}\right) \operatorname{and} \log (g)(\mathrm{cf}$. Fig. 1 and Table 1$)$, the theoretical amplitude ratios for modes with $\ell=1,2,3$, and 4 are represented with a red dashed, a green dash-dotted, a blue dotted, and a cyan dash-dot-dot-dotted line, respectively. The dots indicate the observed amplitude ratios and their standard error.
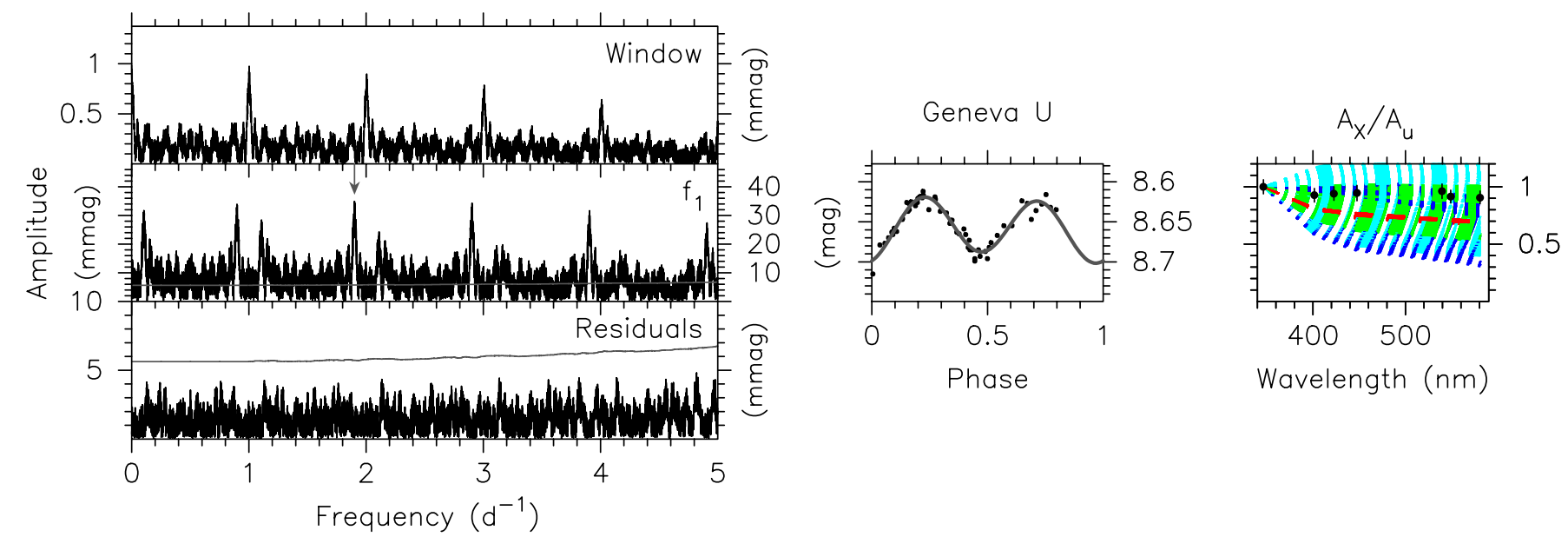

Fig. 24. Same as Fig. 23, but for $f_{\text {orb }}=0.95053(14) \mathrm{d}^{-1}$ and $2 f_{\text {orb }}$ in the GENEVA $U$ passband of HD 24094 .
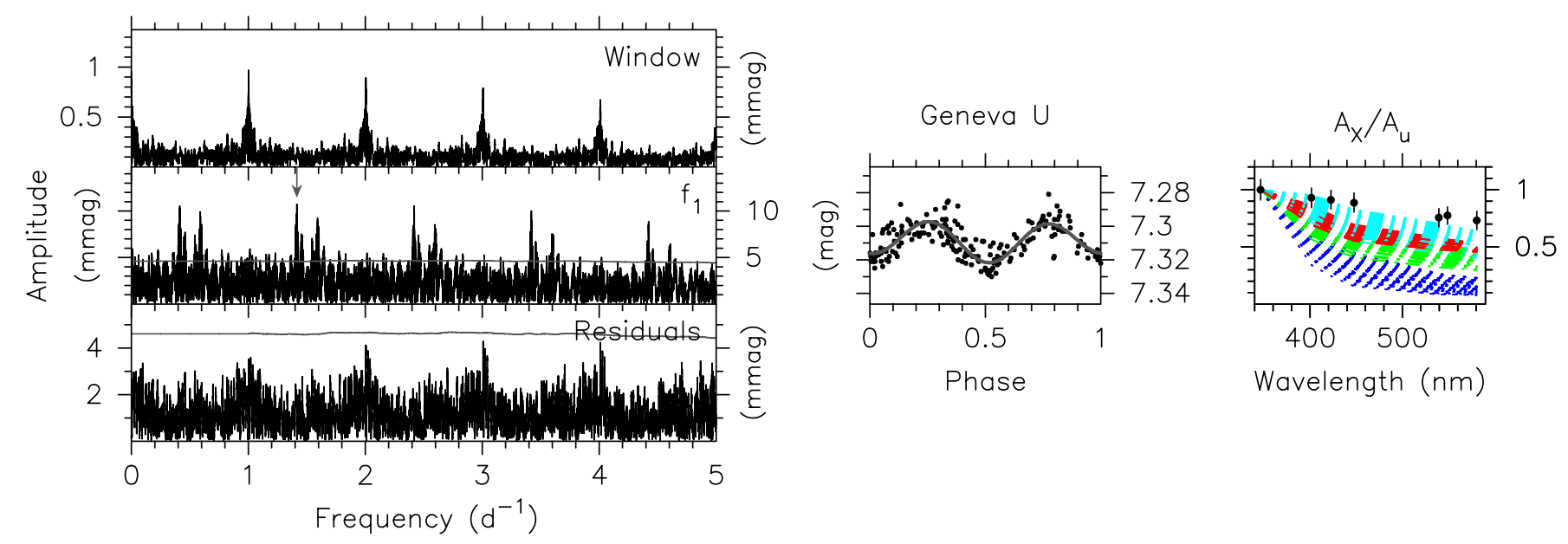

Fig. 25. Same as Fig. 23, but for $f_{\text {orb }}=0.70745(21) \mathrm{d}^{-1}$ and $2 f_{\text {orb }}$ in the GENEVA $U$ passband of HD 112396 .

frequency. After optimisation, we find $f_{\Omega}=1.95767(110) \mathrm{d}^{-1}$ with the MERCATOR and HIPPARCOS data. In the MERCATOR data, up to the second harmonic $\left(2 f_{\Omega}, 3 f_{\Omega}\right)$ is significant, while only the first harmonic is significant in the HIPPARCOS data. 
P. De Cat et al.: Long term photometric monitoring with the Mercator telescope, Online Material p 17
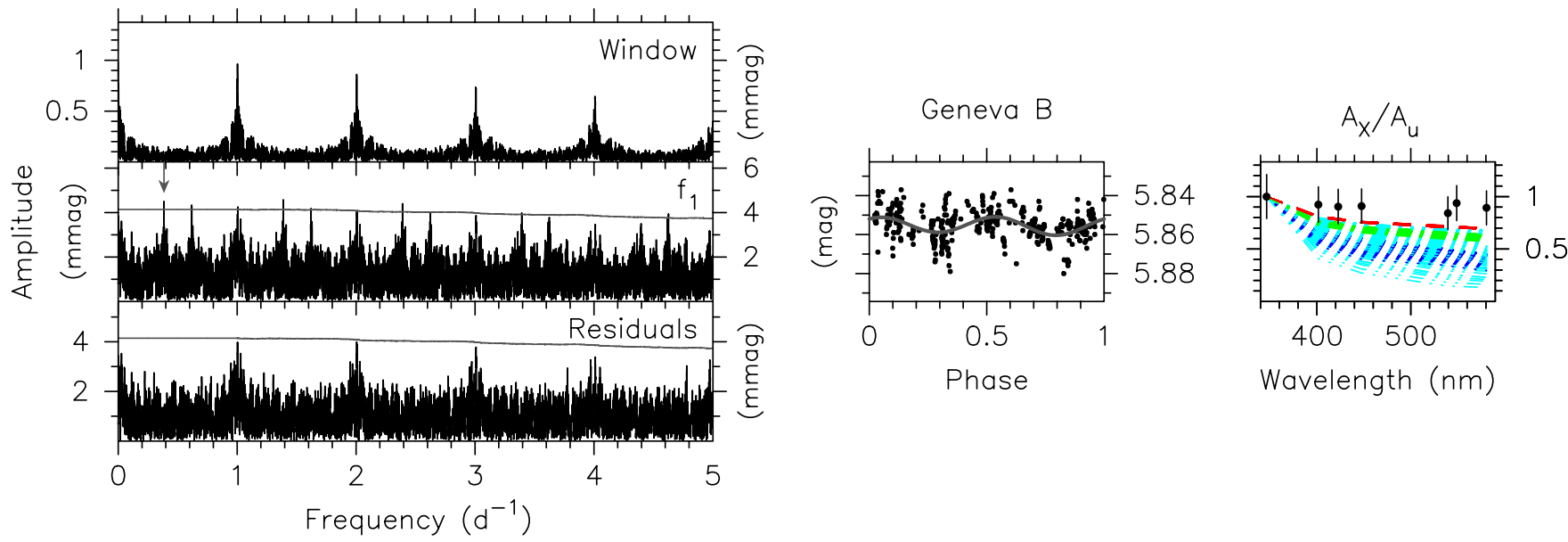

Fig. 26. Same as Fig. 23, but for $f_{\text {orb }}=0.19223(53) \mathrm{d}^{-1}$ and $2 f_{\text {orb }}$ in the GENEVA $B$ passband of HD 149881 .
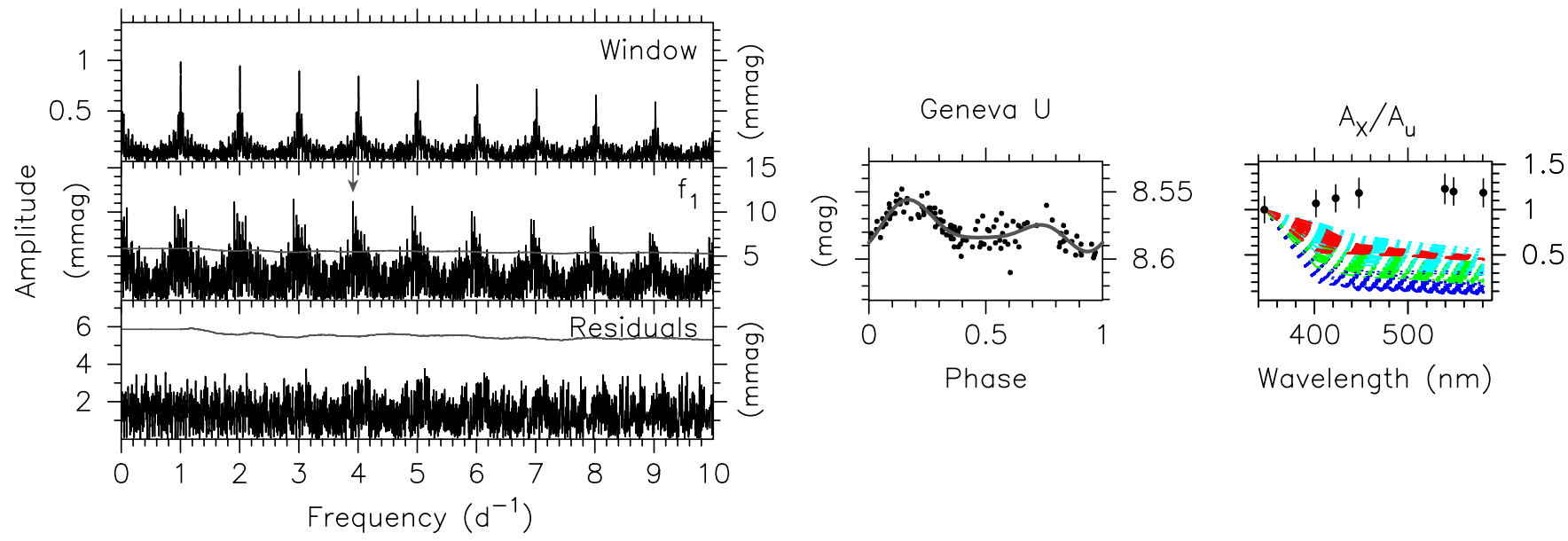

Fig. 27. Frequency analysis of the data of HD 154689 in the GENEVA $U$ passband. In the left panels, we show the window function (top), and the modified SCARGLE periodograms before and after prewhitening with $f_{\Omega}=1.95767(110) \mathrm{d}^{-1}, 2 f_{\Omega}$, and $3 f_{\Omega}$. The dominant frequency $f_{1}=2 f_{\Omega}$ is indicated with an arrow. The grey line corresponds to the $3.6 \mathrm{~S} / \mathrm{N}$-level. In the middle panel, we show a phase diagram with $f_{\Omega}$. The dots and the grey full line denote the observations and the harmonic fit, respectively. The right panel shows the results of the interpretation of the dominant frequency in terms of pulsations. For each theoretical model within the observed range of $\log \left(T_{\text {eff }}\right) \operatorname{and} \log (g)$ (cf. Fig. 1 and Table 1$)$, the theoretical amplitude ratios for modes with $\ell=1,2,3$, and 4 are represented with a red dashed, a green dash-dotted, a blue dotted, and a cyan dash-dot-dot-dotted line, respectively. The dots indicate the observed amplitude ratios and their standard error.
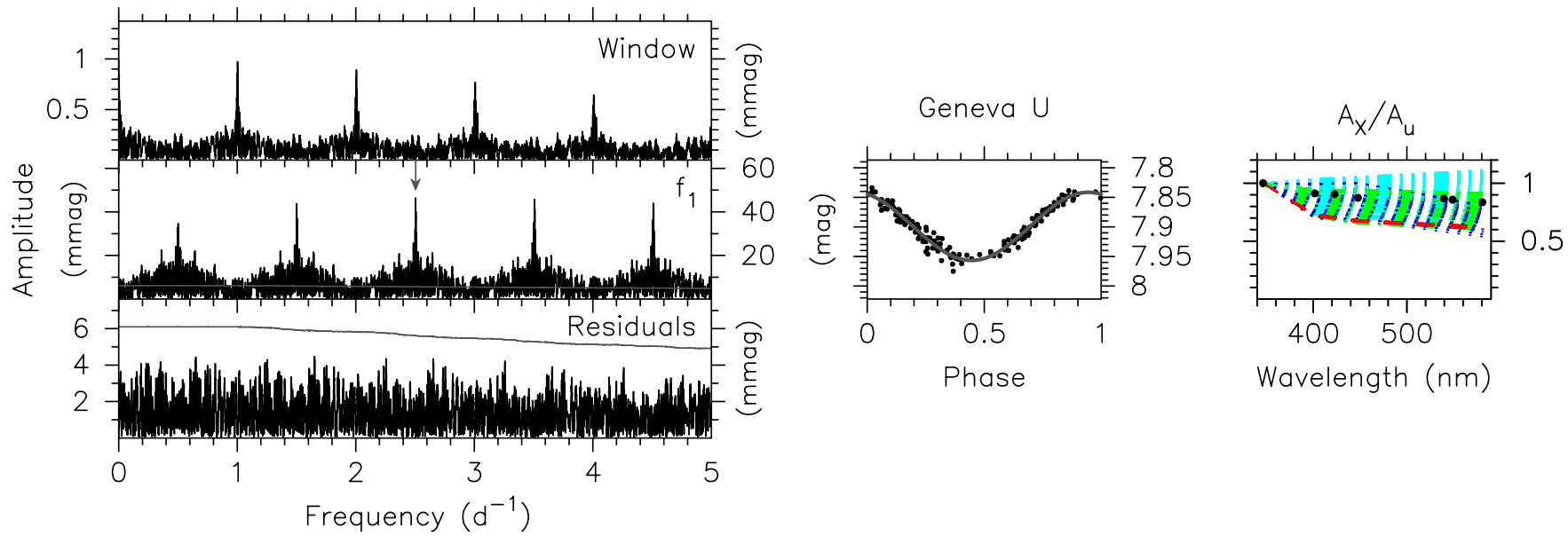

Fig. 28. Same as Fig. 27, but for $f_{1}=2.50476(2) \mathrm{d}^{-1}$ in the GENEVA $U$ passband of HD 46005 .

Light curves of spotted stars vary in almost all cases with at least two harmonics (North 1984; Briquet et al. 2004). There is no evidence for additional frequencies in the residual signal.
In the SIMBAD Astronomical database, the Maia candidate HD 46005 (HIP 31065, V727 Mon) is classified as an eclipsing binary of the $\beta$ Lyrae type. It is known as a visual binary with 
P. De Cat et al.: Long term photometric monitoring with the Mercator telescope, Online Material p 18

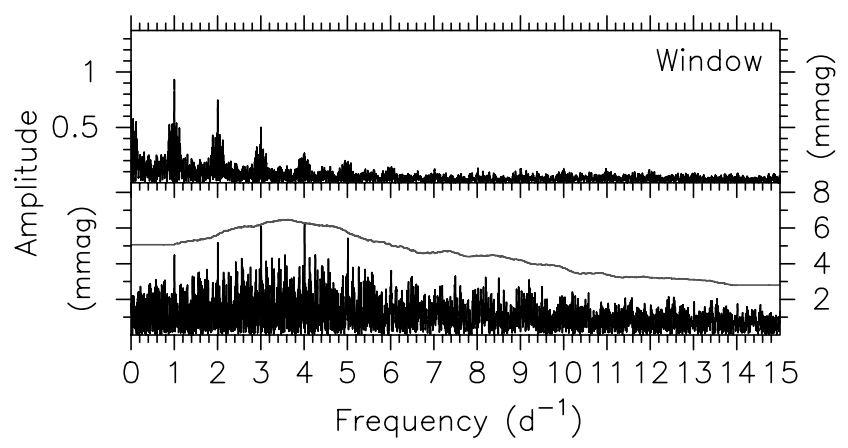

Fig. 29. Frequency analysis of the data of HD 217782 in the GENEVA $U$ passband. We show the window function (top) and the modified SCARGLE periodograms (bottom). The grey line corresponds to the $3.6 \mathrm{~S} / \mathrm{N}-$ level.

$\rho=11^{\prime \prime} 075(20)$ and $\triangle m=2.40(13)$ mag at the epoch of the HIPPARCOS observations (ESA 1997). Therefore, the stellar parameters derived from the GENEVA photometry should be treated with caution (Table 1 ). They are not compatible with the classification as a B8-type star (SIMBAD), and put this object below the cool common part of the theoretical instability strips of the SPBs and $\beta$ Ceps (leftmost star in Fig. 1). The main frequency in the MERCATOR and HIPPARCOS data coincide. We found $f_{1}=2.50476(2) \mathrm{d}^{-1}$ (Fig. 28). This frequency reduces the variance with $90 \%$ or more in all the GENEVA passbands and it is present in the variations of the GENEVA colours. There is no evidence for additional frequencies. The variations with $f_{1}$ can be successfully modelled with an $\ell=2$ mode (Fig. 28, right panel). However, the observed photometric amplitudes are extremely large for an $\ell=2$ mode: from 57.7(10) mmag in $U$ to $48.2(12) \mathrm{mmag}$ in $G$ (Table 2) and 50.2(24) mmag in $H_{\mathrm{p}}$ (Table 3). This is more than $50 \%$ larger than what is observed for the large-amplitude rapidly-rotating $\beta$ Cep star HD 203664 (Aerts et al. 2006). Moreover, the frequency $f_{1}$ is high for an SPB-frequency and low for a $\beta$ Cep-frequency, and there are no unstable modes with eigenfrequencies in this frequency range for appropriate models. We therefore propose surface spots with $f_{1}$ or one of its subharmonics as rotation frequency as an alternative interpretation. However, we cannot exclude binarity.

\subsection{Constant star}

The A-star HD 217782 (HIP 113788, 2 And) is known as a $\beta$ Pictoris-like object. Both an interstellar cloud in the line-ofsight and in-falling circumstellar gas are observed (Cheng \& Neff 2003 and references therein). Unlike $\beta$ Pictoris, there is no evidence for circumstellar dust nor chromospheric activity. HD 217782 was first classified as a $\lambda$ Bootis star (i.e. a metalpoor Population I A-type star) by Parenago (1958), but this classification was later questioned by Gerbaldi et al. (2003). Indeed, HD 217782 is a visual binary for which Kamper (1974) found an eccentric orbit of 74 years. They could not confirm the identification of the A component as a suspected spectroscopic binary by Plasket et al. (1921), but the evidence for light variations of the B component first stated by van den Bos (1960) remained. Given the low value of the angular separation and magnitude difference between the two visual components $(\rho=0$ !' 387 and $\triangle m=2.17 \mathrm{mag}$ at the epoch of the HIPPARCOS observations; ESA 1997), spectroscopic observations of HD 217782 result in composite spectra, so that the metal lines may appear too weak and mimic those formed in a single metal deficient atmosphere. Like HD 19374, HD 217782 is listed as one of the least variable stars observed with the HIPPARCOS satellite (Adelman 2001). The MERCATOR data give no evidence for periodic variations since the obvious peaks seen in the modified SCARGLE periodograms all correspond to frequencies invoked by the time sampling (Fig. 29). We classify HD 217782 as photometrically constant to better than 6 mmag. 